OPTIMAL CENSORING SCHEMES

FOR NON-PARAMETRIC INTERVALS 


\title{
OPTIMAL PROGRESSIVE TYPE-II CENSORING SCHEMES FOR NON-PARAMETRIC CONFIDENCE INTERVALS OF QUANTILES
}

\author{
By \\ DONGHOON HAN, B.Sc.
}

\author{
A Project \\ Submitted to the School of Graduate Studies \\ in Partial Fulfilment of the Requirements \\ for the Degree \\ Master of Science
}

McMaster University

(C) Copyright by Donghoon Han, September 2006 
MASTER OF SCIENCE (2006)

(Statistics)
McMaster University

Hamilton, Ontario

TITLE:

Optimal Progressive Type-II Censoring

Schemes for Non-parametric

Confidence Intervals of Quantiles

AUTHOR:

Donghoon Han, B.Sc. (McMaster University)

SUPERVISOR:

Professor N. Balakrishnan

NUMBER OF PAGES: xii, 84 


\section{ABSTRACT}

In this work, optimal censoring schemes are investigated for the non-parametric confidence intervals of population quantiles under progressive Type-II right censoring. The proposed inference can be universally applied to any probability distributions for continuous random variables. By using the interval mass as an optimality criterion, the optimization process is also independent of the actual observed values from a sample as long as the initial sample size $n$ and the number of observations $m$ are predetermined. This study is based on the fact that each (uncensored) order statistic observed from progressive Type-II censoring can be represented as a mixture of underlying ordinary order statistics with exactly known weights $[11,12]$. Using several sample sizes combined with various degrees of censoring, the results of the optimization are tabulated here for a wide range of quantiles with selected levels of significance (i.e., $\alpha=0.01,0.05,0.10)$. With the optimality criterion under consideration, the efficiencies of the worst progressive Type-II censoring scheme and ordinary Type-II censoring scheme are also examined in comparison with the best censoring scheme obtained for a given quantile with fixed $n$ and $m$. 
$K E Y$ WORDS : confidence interval, interval mass, mixture representation, non-parametric inference, optimal censoring scheme, order statistic, progressive Type-II censoring, quantile 


\section{ACKNOWLEDGEMENTS}

First of all, I would like to express my sincere thanks to my supervisor, Dr. N. Balakrishnan without whose supervision, guidance, patience, and encouragement, this project would not have been possible. As well, I would like to thank my family whose constant love and invaluable support have helped me to continue my studies.

In addition to my supervisor, I would also like to extend my appreciation to Dr. R. Viveros-Aguilera and Dr. I. Akhundov for serving on my supervisory committee, and lastly, I would like to thank the Department of Mathematics and Statistics at McMaster University for providing a gracious learning environment and computing facilities. 


\section{Contents}

$\begin{array}{ll}\text { ABSTRACT } & \text { iii }\end{array}$

ACKNOWLEDGEMENTS $\quad$ v

1 INTRODUCTION 1

2 NON-PARAMETRIC CONFIDENCE INTERVALS FOR QUANTILES

3 PROGRESSIVE TYPE-II CENSORING 9

4 CONFIDENCE INTERVALS

USING MIXTURE REPRESENTATIONS

4.1 MIXTURE WEIGHTS OF

UNCENSORED ORDER STATISTICS . . . . . . . . . . . . . 13

4.2 CONFIDENCE INTERVALS UNDER

PROGRESSIVE TYPE-II CENSORING $\ldots \ldots \ldots \ldots$ 
5 OPTIMAL PROGRESSIVE CENSORING SCHEMES

5.1 OPTIMALITY CRITERION AND OPTIMAL SCHEMES . . . . . . 20

5.2 NUMERICAL STUDY . . . . . . . . . . . . . . . . . . . . 24

6 CONCLUSION $\quad 27$

A Results of Numerical Study 33

B Partial R Codes for Numerical Study $\quad 75$

$\begin{array}{ll}\text { C Partial FORTRAN Codes for Numerical Study } & 79\end{array}$ 


\section{List of Tables}

A.1 Optimal PC Schemes for Non-Parametric CIs of $\xi_{0.45}$ with $n=10$ and $m=734$

A.2 Optimal PC Schemes for Non-Parametric CIs of $\xi_{0.35}$ with $n=15$ and $m=635$

A.3 Optimal PC Schemes for Non-Parametric CIs of $\xi_{0.35}$ with $n=15$ and $m=1035$

A.4 Optimal PC Schemes for Non-Parametric CIs of $\xi_{0.50}$ with $n=15$ and $m=1036$

A.5 Optimal PC Schemes for Non-Parametric CIs of $\xi_{0.25}$ with $n=20$ and $m=636$

A.6 Optimal PC Schemes for Non-Parametric CIs of $\xi_{0.25}$ with $n=20$ and $m=1037$

A.7 Optimal PC Schemes for Non-Parametric CIs of $\xi_{0.35}$ with $n=20$ and $m=1037$

A.8 Optimal PC Schemes for Non-Parametric CIs of $\xi_{0.50}$ with $n=20$ and $m=1038$

A.9 Optimal PC Schemes for Non-Parametric CIs of $\xi_{0.25}$ with $n=20$ and $m=1638$

A.10 Optimal PC Schemes for Non-Parametric CIs of $\xi_{0.35}$ with $n=20$ and $m=1639$

A.11 Optimal PC Schemes for Non-Parametric CIs of $\xi_{0.50}$ with $n=20$ and $m=1639$

A.12 Optimal PC Schemes for Non-Parametric CIs of $\xi_{0.65}$ with $n=20$ and $m=1640$

A.13 Optimal PC Schemes for Non-Parametric CIs of $\xi_{0.25}$ with $n=25$ and $m=541$

A.14 Optimal PC Schemes for Non-Parametric CIs of $\xi_{0.25}$ with $n=25$ and $m=1041$ 
A.15 Optimal PC Schemes for Non-Parametric CIs of $\xi_{0.35}$ with $n=25$ and $m=1042$

A.16 Optimal PC Schemes for Non-Parametric CIs of $\xi_{0.50}$ with $n=25$ and $m=1042$

A.17 Optimal PC Schemes for Non-Parametric CIs of $\xi_{0.25}$ with $n=25$ and $m=1543$

A.18 Optimal PC Schemes for Non-Parametric CIs of $\xi_{0.35}$ with $n=25$ and $m=1543$

A.19 Optimal PC Schemes for Non-Parametric CIs of $\xi_{0.50}$ with $n=25$ and $m=1544$

A.20 Optimal PC Schemes for Non-Parametric CIs of $\xi_{0.65}$ with $n=25$ and $m=1544$

A.21 Optimal PC Schemes for Non-Parametric CIs of $\xi_{0.25}$ with $n=25$ and $m=2045$

A.22 Optimal PC Schemes for Non-Parametric CIs of $\xi_{0.35}$ with $n=25$ and $m=2045$

A.23 Optimal PC Schemes for Non-Parametric CIs of $\xi_{0.50}$ with $n=25$ and $m=2046$

A.24 Optimal PC Schemes for Non-Parametric CIs of $\xi_{0.65}$ with $n=25$ and $m=2046$

A.25 Optimal PC Schemes for Non-Parametric CIs of $\xi_{0.75}$ with $n=25$ and $m=2047$

A.26 Optimal PC Schemes for Non-Parametric CIs of $\xi_{0.15}$ with $n=30$ and $m=847$

A.27 Optimal PC Schemes for Non-Parametric CIs of $\xi_{0.25}$ with $n=30$ and $m=848$

A.28 Optimal PC Schemes for Non-Parametric CIs of $\xi_{0.35}$ with $n=30$ and $m=849$

A.29 Optimal PC Schemes for Non-Parametric CIs of $\xi_{0.15}$ with $n=30$ and $m=2549$

A.30 Optimal PC Schemes for Non-Parametric CIs of $\xi_{0.25}$ with $n=30$ and $m=2550$

A.31 Optimal PC Schemes for Non-Parametric CIs of $\xi_{0.35}$ with $n=30$ and $m=2550$

A.32 Optimal PC Schemes for Non-Parametric CIs of $\xi_{0.50}$ with $n=30$ and $m=2551$

A.33 Optimal PC Schemes for Non-Parametric CIs of $\xi_{0.65}$ with $n=30$ and $m=2551$

A.34 Optimal PC Schemes for Non-Parametric CIs of $\xi_{0.75}$ with $n=30$ and $m=2552$ 
A.35 Optimal PC Schemes for Non-Parametric CIs of $\xi_{0.15}$ with $n=35$ and $m=752$

A.36 Optimal PC Schemes for Non-Parametric CIs of $\xi_{0.25}$ with $n=35$ and $m=753$

A.37 Optimal PC Schemes for Non-Parametric CIs of $\xi_{0.15}$ with $n=35$ and $m=3053$

A.38 Optimal PC Schemes for Non-Parametric CIs of $\xi_{0.25}$ with $n=35$ and $m=3054$

A.39 Optimal PC Schemes for Non-Parametric CIs of $\xi_{0.35}$ with $n=35$ and $m=3054$

A.40 Optimal PC Schemes for Non-Parametric CIs of $\xi_{0.50}$ with $n=35$ and $m=3055$

A.41 Optimal PC Schemes for Non-Parametric CIs of $\xi_{0.65}$ with $n=35$ and $m=30 \ldots \ldots \ldots \ldots \ldots$

A.42 Optimal PC Schemes for Non-Parametric CIs of $\xi_{0.75}$ with $n=35$ and $m=3057$

A.43 Optimal PC Schemes for Non-Parametric CIs of $\xi_{0.15}$ with $n=40$ and $m=657$

A.44 Optimal PC Schemes for Non-Parametric CIs of $\xi_{0.25}$ with $n=40$ and $m=658$

A.45 Optimal PC Schemes for Non-Parametric CIs of $\xi_{0.15}$ with $n=40$ and $m=3558$

A.46 Optimal PC Schemes for Non-Parametric CIs of $\xi_{0.25}$ with $n=40$ and $m=3559$

A.47 Optimal PC Schemes for Non-Parametric CIs of $\xi_{0.35}$ with $n=40$ and $m=3559$

A.48 Optimal PC Schemes for Non-Parametric CIs of $\xi_{0.50}$ with $n=40$ and $m=3560$

A.49 Optimal PC Schemes for Non-Parametric CIs of $\xi_{0.65}$ with $n=40$ and $m=3560$

A.50 Optimal PC Schemes for Non-Parametric CIs of $\xi_{0.75}$ with $n=40$ and $m=3561$

A.51 Optimal PC Schemes for Non-Parametric CIs of $\xi_{0.85}$ with $n=40$ and $m=3561$

A.52 Optimal PC Schemes for Non-Parametric CIs of $\xi_{0.15}$ with $n=45$ and $m=662$

A.53 Optimal PC Schemes for Non-Parametric CIs of $\xi_{0.25}$ with $n=45$ and $m=662$ 
A.54 Optimal PC Schemes for Non-Parametric CIs of $\xi_{0.15}$ with $n=45$ and $m=4063$

A.55 Optimal PC Schemes for Non-Parametric CIs of $\xi_{0.25}$ with $n=45$ and $m=4064$

A.56 Optimal PC Schemes for Non-Parametric CIs of $\xi_{0.35}$ with $n=45$ and $m=4064$

A.57 Optimal PC Schemes for Non-Parametric CIs of $\xi_{0.50}$ with $n=45$ and $m=4065$

A.58 Optimal PC Schemes for Non-Parametric CIs of $\xi_{0.65}$ with $n=45$ and $m=4065$

A.59 Optimal PC Schemes for Non-Parametric CIs of $\xi_{0.75}$ with $n=45$ and $m=4066$

A.60 Optimal PC Schemes for Non-Parametric CIs of $\xi_{0.85}$ with $n=45$ and

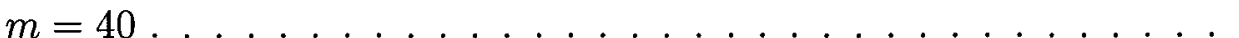

A.61 Optimal PC Schemes for Non-Parametric CIs of $\xi_{0.15}$ with $n=50$ and $m=668$

A.62 Optimal PC Schemes for Non-Parametric CIs of $\xi_{0.25}$ with $n=50$ and $m=668$

A.63 Optimal PC Schemes for Non-Parametric CIs of $\xi_{0.10}$ with $n=50$ and $m=4569$

A.64 Optimal PC Schemes for Non-Parametric CIs of $\xi_{0.15}$ with $n=50$ and $m=4569$

A.65 Optimal PC Schemes for Non-Parametric CIs of $\xi_{0.25}$ with $n=50$ and $m=4570$

A.66 Optimal PC Schemes for Non-Parametric CIs of $\xi_{0.35}$ with $n=50$ and $m=4570$

A.67 Optimal PC Schemes for Non-Parametric CIs of $\xi_{0.50}$ with $n=50$ and $m=4571$

A.68 Optimal PC Schemes for Non-Parametric CIs of $\xi_{0.65}$ with $n=50$ and $m=4571$

A.69 Optimal PC Schemes for Non-Parametric CIs of $\xi_{0.75}$ with $n=50$ and $m=4572$

A.70 Optimal PC Schemes for Non-Parametric CIs of $\xi_{0.85}$ with $n=50$ and

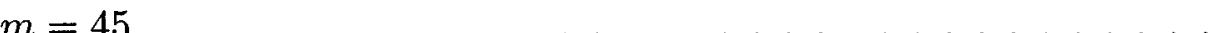


A.71 Optimal PC Schemes for Non-Parametric CIs of $\xi_{0.90}$ with $n=50$ and

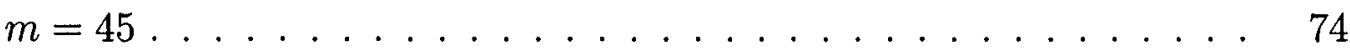




\section{Chapter 1}

\section{INTRODUCTION}

In order to set a warranty period of a new product or even to compare alternative manufacturing designs, the estimation of quantiles is routinely performed in the reliability and lifetime analysis. If one applies a parametric procedure to estimate a quantile, an important presumption underlying the method is that the model fits the data well. Unless this is verified in the very first stage of analysis, the inferential results may lose the power considerably and lead the analyst to a severely distorted conclusion. One way to overcome this obstacle is to apply a non-parametric procedure which does not specify the model structure a priori so that the results of inference are virtually free from the hazard of the model violation. Furthermore, for financial and temporal reasons, censoring is usually unavoidable in practice of a reliability experiment and one special form of intentional censoring to look at is progressive Type-II right censoring. Thus, the primary focus of this project is to review the procedure to construct an exact non-parametric confidence interval for a quantile of interest un- 
der progressive Type-II right censoring and to numerically investigate the associated problem of selecting the optimal censoring schemes using the expected interval mass as an optimality criterion.

During the past decades, the problem of optimal scheduling and optimal censoring has received much attention in different areas of the reliability literature. Balakrishnan and Aggarwala [3] have addressed this problem in general and investigated it using the trace and determinant functions based on the variance-covariance matrix of BLUEs ${ }^{1}$ as optimality criteria for several continuous parametric distributions including exponential, normal, extreme-value, and log-normal. Recently, Gouno, Sen, and Balakrishnan [10] tackled the selection problem of optimal stress change points for a multiple step-stress model when the available data are progressively Type-I censored. In this project, we look at the case of progressive Type-II censoring in a non-parametric setting and the work is based on the results derived by Guilbaud $[11,12]$ on the mixture representation of order statistics from a continuous parent distribution.

Here is the outline of each chapter. Chapter 2 provides a brief overview on non-parametric interval estimation and how to construct it for a given quantile of interest in the complete sample case. In Chapter 3, the concept of censoring is explored, in particular, progressive Type-II right censoring and its mathematical formulation. Chapter 4 then reviews the key results of Guilbaud $[11,12]$ on the mixture representation of order statistics from progressive Type-II censoring and how to derive a confidence interval for a given quantile non-parametrically. In Chapter 5, the issue of finding optimal censoring schemes is discussed and the results of the numerical study

\footnotetext{
${ }^{1}$ best linear unbiased estimators
} 
are tabulated for a range of popular quantiles. Finally, a summary of the results from Chapter 5 along with some suggestions for the future research is provided in Chapter 6. 


\section{Chapter 2}

\section{NON-PARAMETRIC}

\section{CONFIDENCE INTERVALS}

\section{FOR QUANTILES}

Interval estimation for an unknown quantity of interest is an important problem in statistical inference along with hypothesis testing for a decision making process. Unlike a point estimate alone, an interval estimate conveys an indication of reliability and precision of the estimation with a desired level of confidence. A confidence interval provides a range of values within which the true but unknown value of a quantity of interest may lie, and the confidence is gained by constructing such intervals using the same method over and over again [9]. In order to describe the concept of a confidence interval mathematically, let $\hat{\theta}_{L}$ and $\hat{\theta}_{U}$ be functions of a sample $X_{1}, X_{2}, \ldots, X_{n}$ such that 


$$
1-\alpha=\operatorname{Pr}\left[\hat{\theta}_{L} \leq \theta \leq \hat{\theta}_{U}\right]
$$

holds where $1-\alpha$ is referred to as the confidence coefficient and $\theta$ is the unknown quantity of interest one wants to estimate. Then, the interval $\left[\hat{\theta}_{L}, \hat{\theta}_{U}\right]$ is called a twosided confidence interval for $\theta$ with $100(1-\alpha) \%$ level of confidence, and $\hat{\theta}_{L}$ and $\hat{\theta}_{U}$ are called the lower and upper confidence limits, respectively. One can also construct one-sided confidence intervals in a similar manner. Note that both endpoints of the confidence interval are random variables as they are functions of random variables. Thus, the length and location of the interval are random in nature and no one can be sure whether the unknown quantity $\theta$ will actually fall within the confidence limits estimated from a given sample. The objective is then to generate an interval which is as narrow as possible but still includes $\theta$ with an acceptable level of probability.

For a parametric model, the unknown quantities of interest for the point or interval estimations are usually location, scale, and shape parameters as they summarize the distributional information of a parametric model and characterize its behaviour. For an instance, a confidence interval for $\mu$, the population mean of a normal distribution can be easily formulated using a pivotal quantity method depending on whether $\sigma$, the population standard deviation is known or not. For other probability distributions, however, the method of constructing confidence intervals may not be straightforward (e.g., [2]) and one may have to employ a variety of different techniques to obtain the approximate results (e.g., the bootstrap methods).

Another issue concerned with the interval estimation for any types of unknown quantities solely based on a parametric model is the validity of the distribution model 
from the very first stage of analysis. Parametric statistics has earned considerable popularity and wide applicability with its robustness and power to analyze data using distribution models indexed by few parameters. Despite the popularity of the parametric approaches, however, the validity of all the related inferential procedures and results intrinsically relies on the validity of the model being assumed. Therefore, the power of any parametric techniques associated with a particular parametric model is at stake when the model assumption is clearly violated. On the other hand, non-parametric statistics does not depend on the choice of a model and with minimal assumptions, its properties and inferential techniques are virtually distribution-free or model-free. Hence, if one doubts about the adequacy of a parametric model and there are no suitable ways to assess the model validity, non-parametric methods work as an alternative.

Here we illustrate a simple non-parametric procedure to construct the confidence intervals for quantiles based on complete observations from a sample (viz., no censoring). Quantiles of the underlying population distribution are often the quantities one is interested in estimating as they give an insight into the way the population is distributed. Some well-known special quantiles are median, quartiles, centiles, percentiles, etc. These quantiles are used to group the population being studied or to quote some important characteristics of the population. For example, median and quartiles are used to draw the box-and-whisker plots and to describe a data set instead of sample mean and sample standard deviation when the data in hand exhibit a highly skewed distribution. For a model checking purpose, the normal quantile plots are commonly used to assess graphically whether it is safe to assume that the data set in question comes from a normal distribution. In a clinical trial or a medical study, 
quantiles are frequently used to summarize the survival data, and in a reliability experiment or a lifetime analysis, quantiles are also used to evaluate the performance of a testing unit.

Now, in order to construct a non-parametric confidence interval for a given quantile from a continuous distribution $F(t)=\operatorname{Pr}[X \leq t]$, let $\xi_{p}$ be the quantile that satisfies

$$
\operatorname{Pr}\left[X \leq \xi_{p}\right]=F\left(\xi_{p}\right)=p, \quad 0<p<1
$$

where $X$ is a random variable whose $\mathrm{cdf}$ is $F(t)$. Then, suppose that a sample of size $n$ is taken from this population and ordered so that $X_{1}<X_{2}<\ldots<X_{n}$ denote such order statistics from the sample. The probability of the interval $\left(-\infty, X_{j}\right]$ covering $\xi_{p}$ is then given by the lower tail binomial probability,

$$
\begin{aligned}
\operatorname{Pr}\left[X_{j}>\xi_{p}\right] & =\operatorname{Pr}\left[X_{j} \geq \xi_{p}\right] \\
& =\sum_{k=0}^{j-1}\left(\begin{array}{l}
n \\
k
\end{array}\right) p^{k}(1-p)^{n-k}=b_{j}
\end{aligned}
$$

for each $j=1,2, \ldots, n$. Using (2.3), for integers $r$ and $s$ which satisfy $1 \leq r<s \leq n$, the probability of $X_{r} \leq \xi_{p} \leq X_{s}$ is determined by

$$
\begin{aligned}
\operatorname{Pr}\left[X_{r} \leq \xi_{p} \leq X_{s}\right] & =\operatorname{Pr}\left[X_{s} \geq \xi_{p}\right]-\operatorname{Pr}\left[X_{r}>\xi_{p}\right]=b_{s}-b_{r} \\
& =\sum_{k=r}^{s-1}\left(\begin{array}{l}
n \\
k
\end{array}\right) p^{k}(1-p)^{n-k}
\end{aligned}
$$

For a selected confidence coefficient $1-\alpha$, the values of $r$ and $s$ can be searched so 
that $\operatorname{Pr}\left[X_{r} \leq \xi_{p} \leq X_{s}\right] \geq 1-\alpha$ holds. Substituting the observed values $x_{r}$ and $x_{s}$ for $X_{r}$ and $X_{s}$, the two-sided $100(1-\alpha) \%$ confidence interval for $\xi_{p}$ is $\left[x_{r}, x_{s}\right]$. It is remarked that it is not always possible to find $r$ and $s$ to give the conventional 0.90 , 0.95, 0.99 values for $1-\alpha$. Especially with small sample sizes, it gets more difficult to produce a confidence interval non-parametrically with a high level of confidence. Nevertheless, the confidence interval constructed as above is free of any particular forms of the probability distribution $F(t)$ as long as the underlying parent distribution is continuous. 


\section{Chapter 3}

\section{PROGRESSIVE TYPE-II}

\section{CENSORING}

For any statistical analyses, a complete collection of data is the most favorable scenario prior to the actual analysis step as the inference made is considered relatively resistant to the uncertainty. In reality, however, statistical analysts and practitioners frequently encounter situations where the data are not all observable. Then, it is questionable whether comparable inference based on the incomplete sample can be devised to the complete sample case. One type of such incomplete data which arises commonly in practice is censored data. Censored data are observed when the experiments involving lifetimes of testing units have to be terminated earlier than scheduled. For the reasons of cost reduction and time constraint, intentional censoring is unavoidable in practice, especially for a reliability study and a survival analysis. The two traditional forms of censoring which have been studied extensively in the past 
are Type-I and Type-II censoring. Type-I censoring occurs when the experiment is terminated at a prefixed time $T$, independent of the failure times. Within this, Type-I right censoring implies that no failures would be observed beyond this time $T$. While Type-I censoring specifies the time of termination, conventional Type-II censoring restricts the number of failures to be observed. As such, in Type-II right censoring, there would be a prefixed number $m$ so that the experiment is terminated at the time of the $m$ th failure and all the remaining units are removed from the experiment.

In addition, a more general censoring, named progressive censoring, has recently been developed. The concept of progressive censoring was first introduced in 1956 by Herd at Iowa State College in his Ph.D. thesis entitled Estimation of the parameters of a population from a multi-censored sample [3]. The subject was further developed in 1963 by A. C. Cohen [6] and it has received much attention for research since then. The importance of progressive censoring lies in its efficient exploitation of the available resources compared to the traditional sampling. Withdrawn unfailed testing units are typically used in other experiments in the same or at a different facility [10]. Progressive censoring can also be either Type-I or Type-II, and in fact, it includes both the conventional Type-I and Type-II censorings as special cases. Progressive Type-I right censored samples are observed when a pre-specified number or proportion of unfailed units are continuously removed during the experiment at each pre-determined time point. Similarly, progressive Type-II right censored samples arise when a pre-specified number of unfailed units are continuously withdrawn from the experiment at each failure time observed until the pre-determined number of units have failed.

Among various types of censoring, it is the interest in this project to study 
progressive censoring, in particular, progressive Type-II right censoring as it attains more tractable and interesting mathematical properties. Now, let us spell out how progressive Type-II censoring proceeds in terms of the underlying ordinary order statistics. Consider a life-testing experiment involving $n$ experimental units and $m$ failures are to be observed with $2 \leq m \leq n$. Besides, let $\mathbf{R}=\left(R_{1}, R_{2}, \ldots, R_{m}\right)$ be the planned progressive censoring scheme of one's choice which satisfies $R_{i} \geq 0$ for $i=1,2, \ldots, m$ and

$$
\sum_{i=1}^{m} R_{i}+m=n
$$

The failure times of the testing units since time zero can be viewed as a random sample of size $n$ from a random variable $X$ with cdf $F(t)$, and the corresponding order statistics of the successive failure times are denoted by $X_{1}<X_{2}<\cdots<X_{n}$. As the parent distribution $F(t)$ is assumed to be continuous, $X_{1}, X_{2}, \ldots, X_{n}$ are distinct with probability 1 . Nevertheless, $X_{1}, X_{2}, \ldots, X_{n}$ may not be all observable because of progressive Type-II censoring outlined as follows.

Right after observing the first failure at time $Y_{1}\left(\equiv X_{1}\right), R_{1}$ of the $N_{1}=n-1$ surviving units are selected at random (without replacement) and removed from the experiment so that only $n_{1}=N_{1}-R_{1}$ units remain under observation. Immediately after the second failure at time $Y_{2}, R_{2}$ of the $N_{2}=n_{1}-1$ surviving units are selected at random (without replacement) and removed so that only $n_{2}=N_{2}-R_{2}$ units remain under observation, and so on. Hence, for $i=1,2, \ldots, m-1, N_{i}=n_{i-1}-1$ denotes the number of units surviving beyond $Y_{i}$ just before $R_{i}$ units are removed and $n_{i}=N_{i}-R_{i}$ denotes the number of units remaining beyond $Y_{i}$ right after $R_{i}$ 
units are removed, with $n_{0}=n$. It is also defined that $N_{m}=n_{m-1}-1=R_{m}$ and $n_{m}=0$ since all $N_{m}$ units left after the $m$ th failure at time $Y_{m}$ are withdrawn from the experiment if there are any. Therefore, all $N_{i}$ 's and $n_{i}$ 's are determined by the censoring scheme $\mathbf{R}$. The progressive Type-II censoring just described leads to $m$ observable uncensored order statistics $Y_{1}<Y_{2}<\cdots<Y_{m}$ that are available for inference. As mentioned before, the conventional Type-II right censoring is a special case with $R_{1}=R_{2}=\cdots=R_{m-1}=0$ and $R_{m}=n-m>0$, whereas the complete sample case (viz., no censoring) corresponds to the case with $m=n$ and $R_{1}=R_{2}=\cdots=R_{m}=0$. 


\section{Chapter 4}

\section{CONFIDENCE INTERVALS \\ USING MIXTURE}

\section{REPRESENTATIONS}

\subsection{MIXTURE WEIGHTS OF}

\section{UNCENSORED ORDER STATISTICS}

Recently, Guilbaud $[11,12]$ derived how each observed uncensored order statistic $Y_{1}<Y_{2}<\cdots<Y_{m}$ can be represented as a mixture of underlying ordinary order statistics $X_{1}<X_{2}<\cdots<X_{n}$ under progressive Type-II right censoring. In order to summarize the results, let $\mathbf{Y}=\left(Y_{1}, Y_{2}, \ldots, Y_{m}\right)^{T}$ and $\mathbf{X}=\left(X_{1}, X_{2}, \ldots, X_{n}\right)^{T}$. Also, let $\hat{w}_{i, j}$ be the indicator of the event $Y_{i}=X_{j}$, which equals 1 if the event occurs and 0 otherwise. Hence, $\hat{w}_{i, j}$ 's simply tell which of the order statistics $X_{1}<X_{2}<\cdots<X_{n}$ 
is selected as one of $Y_{1}<Y_{2}<\cdots<Y_{m}$. Then, the $m \times n$ random matrix $\hat{W}=\left(\hat{w}_{i, j}\right)$ composed of the indicators holds the relationship of

$$
\mathbf{Y}=\hat{W} \mathbf{X}
$$

with $\hat{w}_{1,1}=1$ since $Y_{1}=X_{1}$ by definition. Obviously, $\hat{W}$ has a structural property of unit row sums. Moreover, for $2 \leq i \leq m, \hat{w}_{i, j}=0$ for $j<i$ and the column numbers of 1 's are in the strictly increasing order.

Using the idea of sequential and independent simple random sampling without replacement, $\hat{W}$ can be further decomposed into a product of $m$ matrices that are mutually independent of each other and of $\mathbf{X}$. It is expressed by

$$
\hat{W}=K^{(m)} \hat{K}^{(m-1)} \cdots \hat{K}^{(1)}
$$

where $\hat{K}^{(i)}$ is a $\left(i+n_{i}\right) \times\left(i+N_{i}\right)$ matrix defined as

$$
\hat{K}^{(i)}=\left(\begin{array}{c|c}
I^{(i)} & 0_{1}^{(i)} \\
\hline 0_{2}^{(i)} & \hat{H}^{(i)}
\end{array}\right),
$$

for $i=1,2, \ldots, m-1$, and $K^{(m)}$ is a non-random $m \times\left(m+N_{m}\right)$ matrix given by

$$
K^{(m)}= \begin{cases}\left(I^{(m)} \mid 0_{1}^{(m)}\right), & \text { if } N_{m}>0 \\ I^{(m)}, & \text { if } N_{m}=0\end{cases}
$$

Here, $I^{(i)}$ is a $i \times i$ identity matrix and $\hat{H}^{(i)}=\left(\hat{h}_{r, s}^{(i)}\right)$ is the $n_{i} \times N_{i}$ matrix of indicators $\hat{h}_{r, s}^{(i)}$ of the events $a_{r}^{(i)}=b_{s}^{(i)}$, with $a_{1}^{(i)}<\cdots<a_{n_{i}}^{(i)}$ and $b_{1}^{(i)}<\cdots<b_{N_{i}}^{(i)}$ denoting 
the order statistics remaining beyond $Y_{i}$ immediately after/before withdrawing $R_{i}$ surviving units. $0_{1}^{(i)}$ and $0_{2}^{(i)}$ are simply conformable zero matrices. Clearly, the distribution of $\hat{H}^{(i)}$ is determined by $n_{i}$ and $N_{i}$, and the expectation of each $\hat{h}_{r, s}^{(i)}$ is given by

$$
\begin{aligned}
h_{r, s}^{(i)} & =\operatorname{Pr}\left[a_{r}^{(i)}=b_{s}^{(i)}\right] \\
& = \begin{cases}\left(\begin{array}{l}
s-1 \\
r-1
\end{array}\right)\left(\begin{array}{l}
N_{i}-s \\
n_{i}-r
\end{array}\right) /\left(\begin{array}{c}
N_{i} \\
n_{i}
\end{array}\right), & \text { if } r \leq s \leq r+R_{i}, \\
0, & \text { otherwise }\end{cases}
\end{aligned}
$$

for $1 \leq r \leq n_{i}$ and $1 \leq s \leq N_{i}$. As a result, the expectation of $\hat{H}^{(i)}$ equals $H^{(i)}=\left(h_{r, s}^{(i)}\right)$ whose elements are defined by (4.5). Structurally, $H^{(i)}$ is a band matrix with positive elements only if $r \leq s \leq r+R_{i}$ and 0 otherwise. For each row, the sum is exactly 1 , and in the complete sample case (viz., no censoring), $H^{(i)}$ becomes an identity matrix as $n=m$ and $R_{1}=R_{2}=\cdots=0$.

Now, from the independence of the factors in (4.2), it easily follows that the expectation of $\hat{W}$ equals

$$
W=K^{(m)} K^{(m-1)} \cdots K^{(1)}
$$

where $K^{(i)}=E\left[\hat{K}^{(i)}\right]$ for $i=1,2, \ldots, m-1$ and it is the right member of (4.3) with $\hat{H}^{(i)}$ replaced by $H^{(i)}$. The properties of $W=\left(w_{i, j}\right)$ are such that each row sum is 1 and the number of positive elements in the successive rows form a non-decreasing sequence. Consequently, one can see that for each $i=2,3, \ldots, m,\left(\hat{w}_{i, 1}, \hat{w}_{i, 2}, \ldots, \hat{w}_{i, n}\right)$ has multinomial distribution with parameters 1 and $\left(w_{i, 1}, w_{i, 2}, \ldots, w_{i, n}\right)$. Given the 
independence of $\hat{W}$ and $\mathbf{X}$, the mixture representation (4.1) thus holds with the weights $w_{i, j}=\operatorname{Pr}\left[Y_{i}=X_{j}\right]=\operatorname{Pr}\left[\hat{w}_{i, j}=1\right]=E\left[\hat{w}_{i, j}\right]$ for $1 \leq i \leq m$ and $1 \leq$ $j \leq n$, and the mixture weights are given by the non-random matrix $W=E[\hat{W}]$. It is also important to note that $W$ is completely determined by $n, m$, and $\mathbf{R}=$ $\left(R_{1}, R_{2}, \ldots, R_{m}\right)$, and the computation of all the elements of $W$ can be carried out via an efficient recursive relation described in Guilbaud [11].

\subsection{CONFIDENCE INTERVALS UNDER PROGRESSIVE TYPE-II CENSORING}

In Chapter 2, a procedure to construct a non-parametric confidence interval for a quantile $\xi_{p}$ was illustrated in the complete sample case. Using the mixture representation given in (4.1), a non-parametric confidence interval for $\xi_{p}$ under progressive Type-II right censoring can be constructed in a similar way as described in $[11,12]$. Suppose that $\xi_{p}$ is a given $p$-quantile that satisfies (2.2) and it is uniquely defined through $\xi_{p}=F^{-1}(p)$ for any continuous distribution function $F(t)$. It is then our interest here to estimate its two-sided confidence interval non-parametrically under progressive Type-II right censoring. Before proceeding, let $\mathbf{b}=\left(b_{j}\right)$ be the $n \times 1$ matrix whose elements are defined by (2.3) and let $\mathbf{a}=\left(a_{j}\right)$ be the $m \times 1$ matrix whose elements are defined in terms of $W=\left(w_{i, j}\right)$ through 


$$
\mathbf{a}=W \mathbf{b} .
$$

The vector a is simply a collection of the probabilities of covering $\xi_{p}$ by each $Y_{r}$ since

$$
\begin{aligned}
\operatorname{Pr}\left[Y_{r} \geq \xi_{p}\right] & =\operatorname{Pr}\left[Y_{r}>\xi_{p}\right] \\
& =\sum_{j=1}^{n} w_{r, j} \operatorname{Pr}\left[X_{j} \geq \xi_{p}\right] \\
& =\sum_{j=1}^{n} w_{r, j} b_{j}=a_{r},
\end{aligned}
$$

for $r=1,2, \ldots, m$. It is clear that $a_{1} \leq a_{2} \leq \cdots \leq a_{m}$ as $Y_{1}<Y_{2}<\cdots<Y_{m}$ with probability 1 , and in the special case with no censoring, $W$ becomes a $n \times n$ identity matrix, yielding $\mathbf{a}=\mathbf{b}$. Now, suppose that $r$ and $s$ are some integers satisfying $1 \leq r<s \leq m$. The coverage probability of the interval estimator $\left[Y_{r}, Y_{s}\right]$ for $\xi_{p}$ can then be easily expressed in terms of the elements of a through

$$
\operatorname{Pr}\left[Y_{r} \leq \xi_{p} \leq Y_{s}\right]=\operatorname{Pr}\left[Y_{s} \geq \xi_{p}\right]-\operatorname{Pr}\left[Y_{r}>\xi_{p}\right]=a_{s}-a_{r}
$$

Provided a has been evaluated via (4.7), the integers $r$ and $s$ can be determined so that the confidence level of this interval is at least a specified value $1-\alpha$ (i.e., $\left.a_{s}-a_{r} \geq 1-\alpha\right)$. It is recognized here that $\mathbf{a}$ is such that this is feasible. It is also remarked that the confidence interval constructed as described above does not depend on a functional form of $F(t)$ and thus, it is a distribution-free property as long as the underlying parent distribution is continuous.

Since the coverage probability (4.8) is established by the matrix operation of 
(4.7) and it is the final key ingredient to accomplish our goal of finding the interval estimator for $\xi_{p}$ under progressive Type-II censoring, it is worth introducing other efficient way to estimate (4.8) without going through direct computation of the matrix $W$. Using the expression of the explicit density function of $Y_{r}$ given in [4], one can easily calculate (4.8) and this is particularly useful when one wants to estimate only one or few elements of a and to avoid intensive and costly computation of the whole matrix $W$. Adopting the usual conventions that $\prod_{i=1}^{0} u_{i} \equiv 1$ and $\sum_{i=1}^{0} u_{i} \equiv 0$, the density function of $Y_{r}$ is given by

$$
\begin{aligned}
& f_{r: m: n}\left(y_{r}\right)=c \sum_{i=0}^{r-1} c_{i, r-1}\left(R_{1}+1, R_{2}+1, \ldots, R_{r-1}+1\right) f\left(y_{r}\right)\left(1-F\left(y_{r}\right)\right)^{R_{i}^{\prime \prime}-1}, \\
& -\infty<y_{r}<\infty \text { for } r=1,2, \ldots, m, \text { where }
\end{aligned}
$$

$$
\begin{aligned}
c & =n\left(n-R_{1}-1\right) \cdots\left(n-R_{1}-\ldots-R_{r-1}-r+1\right), \\
R_{i}^{\prime \prime} & =n-\sum_{j=1}^{r-i-1}\left(R_{j}+1\right)
\end{aligned}
$$

and $f(t)$ denotes the corresponding pdf of an absolutely continuous distribution function $F(t)$ such that $f(t)=\frac{d}{d t} F(t)$. In addition, $c_{i, r-1}\left(R_{1}+1, R_{2}+1, \ldots, R_{r-1}+1\right)$ is obtained through a function

$$
c_{i, q}\left(\mathbf{u}_{q}\right)=\frac{(-1)^{i}}{\left(\prod_{j=1}^{i} \sum_{k=q-i+1}^{q-i+j} u_{k}\right)\left(\prod_{j=1}^{q-i} \sum_{k=j}^{q-i} u_{k}\right)},
$$

defined for any real vector $\mathbf{u}_{q}=\left(u_{1}, u_{2}, \ldots, u_{q}\right)$ of length $q \geq 1$. Integrating the density function, the corresponding distribution function or the cdf of $Y_{r}$ is given by 


$$
\begin{aligned}
& F_{r: m: n}\left(y_{r}\right)= \int_{-\infty}^{y_{r}} f_{r: m: n}(y) d y \\
&=c \sum_{i=0}^{r-1} c_{i, r-1}\left(R_{1}+1, R_{2}+1, \ldots, R_{r-1}+1\right) R_{i}^{\prime \prime-1} \\
& \quad \times\left(1-\left(1-F\left(y_{r}\right)\right)^{R_{i}^{\prime \prime}}\right),
\end{aligned}
$$

$-\infty<y_{r}<\infty$ for $r=1,2, \ldots, m$. Using the complementary event, the coverage probability (4.8) can be explicitly formulated as

$$
\begin{aligned}
\operatorname{Pr}\left[Y_{r} \geq \xi_{p}\right]= & \operatorname{Pr}\left[Y_{r}>\xi_{p}\right] \\
= & 1-\operatorname{Pr}\left[Y_{r} \leq \xi_{p}\right]=1-F_{r: m: n}\left(\xi_{p}\right) \\
= & 1-c \sum_{i=0}^{r-1} c_{i, r-1}\left(R_{1}+1, R_{2}+1, \ldots, R_{r-1}+1\right) R_{i}^{\prime \prime-1} \\
& \times\left(1-(1-p)^{R_{i}^{\prime \prime}}\right),
\end{aligned}
$$

and the last step results from the definition of $\xi_{p}$ stated in (2.2). 


\section{Chapter 5}

\section{OPTIMAL PROGRESSIVE CENSORING SCHEMES}

\subsection{OPTIMALITY CRITERION}

\section{AND OPTIMAL SCHEMES}

In the previous chapter, the exact non-parametric interval estimator for any given quantile $\xi_{p}$ has been derived when a particular progressive Type-II censoring scheme is to be applied. Then, as pointed out in [3], some natural questions that arise are: "how can a practitioner decide on which censoring scheme to be used out of numerous censoring schemes?" "Is the decision made strictly on the basis of convenience, or can one select a censoring scheme which makes the most sense within some statistical settings?" From a practical point of view, the question of choosing the optimal values for $\mathbf{R}=\left(R_{1}, R_{2}, \ldots, R_{m}\right)$ is certainly an indispensable one and it 
has to be addressed when one designs a progressive Type-II censoring experiment as there are astronomical figures of distinct censoring schemes even for a moderate size of $n$ and $m$.

Before selecting the optimal censoring scheme, one must first devise an optimality criterion or an objective function to be optimized [3]. Consequently, the meaning of the optimal censoring scheme is restricted to the criterion of one's choice. In the case of non-parametric interval estimation for a quantile $\xi_{p}$ with $n$ and $m$ fixed, a simple optimization with respect to the choice of $\mathbf{R}=\left(R_{1}, R_{2}, \ldots, R_{m}\right)$ is to select $\mathbf{R}$ which enables to find $r$ and $s$ in (4.9) that satisfy $a_{s}-a_{r} \approx 1-\alpha$ and $a_{r} \approx 1-a_{s}$. This constraint is equivalent to find $r$ and $s$ satisfying $a_{r} \approx \alpha / 2$ and $a_{s} \approx 1-\alpha / 2$ in order to yield a symmetric confidence interval when it is possible.

In the circumstance without any censoring, a reasonable objective function with respect to the choice of $r$ and $s$ is the index difference $s-r$ corresponding to the relevant interval $\left[X_{r}, X_{s}\right]$ with the level of confidence at least $1-\alpha$. To minimize this function is to minimize the expected probability mass $F\left(X_{s}\right)-F\left(X_{r}\right)$ of the underlying distribution within the interval [7]. This clearly reflects the purpose of the interval estimation, which is to produce the shortest interval with a desired confidence level. Under progressive Type-II right censoring, it follows from (4.1) that $Y_{i}=\sum_{j=1}^{n} \hat{w}_{i, j} X_{j}$ and the expectation of $F\left(Y_{i}\right)=\sum_{j=1}^{n} \hat{w}_{i, j} F\left(X_{j}\right)$ equals

$$
e_{i}=\sum_{j=1}^{n} w_{i, j} \frac{j}{n+1}
$$

for $i=1,2, \ldots, m$. As noted in [11], it is convenient to realize that the $m \times 1$ matrix $\mathbf{e}=\left(e_{i}\right)$ whose elements are defined by $(5.1)$ can be computed by $(n+1)^{-1} W J$ with 
$J=(1,2, \ldots, n)^{T}$. Also, notice that (5.1) depends on the elements of the weight matrix $W$. Even if $W$ is not available, (5.1) can still be computed easily using the equivalent expression derived from the probability integral transformation under progressive Type-II censoring and the generalization of Malmquist's transformation results through the independent ratios from beta distribution [3]. The expression is rather explicit and it is given by

$$
e_{i}=1-\prod_{j=m-i+1}^{m} \frac{\delta_{j}}{\delta_{j}+1}
$$

where

$$
\delta_{j}=j+\sum_{k=m-j+1}^{m} R_{k},
$$

for $i=1,2, \ldots, m$. Therefore, the expectation of the probability mass within the interval $\left[Y_{r}, Y_{s}\right]$ is equal to $e_{s}-e_{r}$ for $1 \leq r<s \leq m$. Then, for given $n$ and $m$, the optimal progressive Type-II censoring scheme for the non-parametric interval estimator of $\xi_{p}$ is obtained by minimizing the expected interval mass

$$
M(\mathbf{R})=\min _{(r, s) \in \mathcal{S}}\left\{e_{s}-e_{r}\right\}
$$

where $\mathcal{S}$ is a subset of binary Cartesian product of positive integers $(r, s)$ such that $1 \leq r<s \leq m$ and $a_{s}-a_{r} \geq 1-\alpha$ under given conditions. If $\mathcal{S}$ is an empty set for some $\mathbf{R}$ 's, $M(\mathbf{R})$ is simply defined to be 1 . It is remarked that the objective function $M(\cdot)$ and the index set $\mathcal{S}$ are both depending on the choices of $n, m, \mathbf{R}$, and $\alpha$ for a given quantile $\xi_{p}$, but $M(\mathbf{R})$ is minimized with respect to every possible 
progressive censoring scheme $\mathbf{R}=\left(R_{1}, R_{2}, \ldots, R_{m}\right)$ under $n, m, \alpha$ all fixed in advance along with $\xi_{p}$. It provides flexibility to the practitioners as the number of units to be put on the test and the number of complete failure times to be observed are both to be determined by a test designer based on the availability of units and experimental facilities. If one or both of these are to be determined in the planning stage, one may also use the tables presented in the next section to decide upon the values of $n$ and $m$ which are feasible given an agreeable value of the objective function.

In this finite sample case, to minimize (5.4) with all the other values given, one may list each and every possible choice of censoring schemes and the corresponding values of the objective function. After determining the best value, the value which minimizes the expected interval mass $M(\cdot)$, or a certain region of satisfactory values from this list, one can pick out either the best censoring scheme or one which gives a value very close to the best but may be practically more convenient (i.e., a suboptimal censoring scheme). As mentioned in [3], for fixed $n$ and $m$, "the increase in efficiencies by employing the optimal progressive Type-II censoring scheme is often substantial compared to the case of conventional Type-II censoring scheme frequently used by practitioners". For the purpose of comparing different censoring schemes with the selected objective function, a sensible definition of the efficiency of a censoring scheme $A, \mathbf{R}_{A}$ with respect to a censoring scheme $B, \mathbf{R}_{B}$ can be given in percentage by

$$
\operatorname{Efficiency}\left(\mathbf{R}_{A}, \mathbf{R}_{B}\right)=\frac{M\left(\mathbf{R}_{A}\right)}{M\left(\mathbf{R}_{B}\right)} \times 100 \%
$$

This is simply a ratio of the interval masses and if one is interested in searching for a region containing a number of satisfactory censoring schemes, such a region 
can be defined in terms of efficiencies [3]. For instance, an experimenter may be pleased with any censoring schemes which are at least $95 \%$ as efficient as the optimal (best) scheme. Then, he can choose the censoring scheme out of these satisfactory ones which is the most practicable or convenient to the experimental setting. Hence, without complicating the calculations, practicality is also built into the objective function by employing a two-stage approach to optimization [3]. Moreover, one can see that for any fixed values of $n$ and $m$, the efficiency of the conventional TypeII censoring scheme with respect to the optimal censoring scheme would be at most $100 \%$. Therefore, "there is no loss in efficiency over the conventional censoring scheme if the optimal scheme is to be used" [3].

\subsection{NUMERICAL STUDY}

Up to now, how to construct a non-parametric confidence interval for a given quantile has been reviewed as well as how to determine the associated optimal progressive Type-II censoring scheme. The weight matrix $W$ can be computed through (4.4) to (4.6) as described in Section 4.1 and the elements (2.3) of $\mathbf{b}$ can be evaluated by the binomial distribution function. Then, an appropriate confidence interval can be searched for a given quantile using the vector a which can be easily estimated from (4.7). Moreover, the vector e can be calculated with the procedure just described in the previous section in order to explore the optimal censoring schemes. 
For illustration and reference for practitioners, a numerical study has been conducted with some selected values of parameters and the results are tabulated in the appendix. The implementation was done using $\mathrm{R}$ and FORTRAN programming languages, and the codes are also provided in the appendix. In the original computation performed, the choices of the sample size $n$ ranged from 10 to 100 with an increment of 5 and the pre-determined number of failure observations $m$ ranged from 2 to $n$ with a unit increment for each choice of $n$. In addition, the choices of $m$ were restricted in such a way that the total number of available censoring schemes $\left(\begin{array}{c}n-1 \\ m-1\end{array}\right)$ does not exceed $3.0 \times 10^{6}$ in order to keep the computational time and space manageable. To examine a variety of quantiles, $p$ of $\xi_{p}$ was selected from 0.05 to 0.95 with an increment of 0.05 . Besides, to yield the conventional $99 \%, 95 \%$, and $90 \%$ confidence intervals, $\alpha$ was chosen to be $0.01,0.05$, and 0.10 , respectively.

Due to a limitation on space, the tables presented in the appendix represent only a portion of the complete results and it is intended to provide the general behaviour of this discrete optimization. Each table is dedicated to a specific quantile with some fixed $n$ and $m$, and it lists the best progressive censoring scheme and the worst censoring scheme along with conventional Type-II censoring scheme for comparison. The best censoring scheme is simply the one which minimizes the objective function $M(\mathbf{R})$ given in (5.4) and the worst censoring scheme is, on the other hand, the one that maximizes

$$
\vec{M}(\mathbf{R})=\max _{(r, s) \in \mathcal{S}}\left\{e_{s}-e_{r}\right\}
$$

whose definition is analogous to (5.4). For some censoring schemes given in the 
tables, the meaning of $0 \star k$ with some positive integer $k$ is to repeat zero $k$ times. Hence, it simply denotes a zero vector of size $k$ embedded in the censoring scheme $\mathbf{R}$. The meaning of $1 \star k$ can be interpreted in a similar way and they are given to dramatically simplify the notation of $\mathbf{R}$. In each table, count denotes the total number of distinct progressive censoring schemes which can produce at least one interval with the confidence level greater than or equal to the nominal level $1-\alpha$. In other words, it is the number of censoring schemes for which $\mathcal{S}$ in (5.4) is a non-empty set. Since the non-parametric confidence interval depends on the order or the index of the uncensored observations, the confidence interval is given in the form of $[r, s]$ from the relevant interval $\left[Y_{r}, Y_{s}\right]$. In each table, the actual level of confidence for each interval estimator is also provided together with its expected probability mass. Additionally, efficiency was calculated using (5.5) with respect to the best censoring scheme found within fixed values of $n, m, p$, and $\alpha$. In the cases where a certain censoring scheme can not yield any intervals with the desired level of confidence, the efficiency is simply noted as not available. 


\section{Chapter 6}

\section{CONCLUSION}

Based on the results of the numerical study presented in Section 5.2 and the appendix, a number of comments can be made on some interesting observations. First of all, it is noted that with certain choices of $n$ and $m$, the available progressive censoring schemes which can generate confidence intervals for a given quantile with a desired level of confidence can be scarce (e.g., Table A.1 and Table A.2). Moreover, the higher the required level of confidence is, the fewer the choices of censoring schemes which can produce such intervals are. One can also observe that for fixed $n, m$, and $\alpha$, the actual level of confidence increases initially and then decreases as $p$ increases. Consequently, when the quantile of interest is too small or too large, there may not be any censoring schemes to generate a confidence interval with a selected level of confidence unless $n$ and $m$ are both reasonably large.

Then, how large should $m$ be compared to $n$ ? It was found that the size of $m$ related to $n$ is also a significant factor to boost up the available censoring schemes 
which can yield confidence intervals with a desired level. By examining tables, it can be seen that if $m$ is too small compared to $n$ or too close to $n$ for fixed $\xi_{p}$ and $\alpha$, the number of censoring schemes which can yield confidence intervals with a desired level decreases. This in turn reduces the number of suboptimal censoring scheme choices whose efficiencies are close to that of the best scheme. To the surprise, this finding also implies that the case without any censoring actually performs worse than the case with an appropriate censoring in constructing a non-parametric confidence interval for a quantile. Therefore, the censoring proportion $\frac{n-m}{n}$ is an important factor to consider at the planning and designing stage of a progressive Type-II censoring experiment if one wants to construct a confidence interval with a desired coverage probability.

Another general observation related to $n$ and $m$ is that raising $n$ with $m$ fixed enables to find the optimal censoring schemes for small quantiles like $\xi_{0.05}$ and $\xi_{0.10}$ within a limited range. On the other hand, increasing $m$ with fixed $n$ enables to find the optimal censoring schemes for a large range of quantiles of interest. Although it is not shown in the tables, it was observed that the maximum level of confidence could be achieved by a censoring scheme $\mathbf{R}=(n-m, 0 \star(m-1))$ in any cases where none of the censoring schemes examined could produce an interval with at least the nominal level. Thus, withdrawing every unit immediately after observing the first failure time is the best in a sense that the interval $\left[Y_{1}, Y_{m}\right]$ attains the highest confidence level. Nevertheless, it turned out to be also the worst censoring scheme in some situations (e.g., Table A.37) when one could locate the optimal censoring scheme. This is because $\left[Y_{1}, Y_{m}\right]$ bears the highest probability mass within the interval.

Although the tables compiled in the appendix can be used for reference for 
statisticians and other practitioners who are planning and designing a progressive Type-II censoring experiment, it is rather difficult to see whether there is a universal pattern or a mathematical trend of the best censoring schemes or the worst censoring schemes. On the contrary, it can be clearly pointed out that if one randomly chooses a progressive censoring scheme for an experiment in which a large censoring takes place, it will be less likely to obtain a desired non-parametric confidence interval for the quantity of interest (e.g., Table A.28). Hence, the thorough planning of an experiment is important regarding the inferential matter. Other interesting observation to point out is that in a few cases, the best censoring scheme coincides with the conventional Type-II censoring scheme. For example, Table A.22 shows that for $n=25$ and $m=20$, the conventional Type-II censoring scheme turns out to be the best censoring scheme to construct a $90 \%$ confidence interval for $\xi_{0.35}$. This result is however valid only with the objective function under consideration and it may not be true for other types of optimality criteria. In most cases, the ordinary Type-II censoring schemes could not even produce a single confidence interval with an acceptable level and the efficiency gained by the optimal censoring scheme was proven to be indeed substantial compared to the conventional Type-II censoring scheme as well as to the worst censoring scheme.

After reviewing the up-to-date literature on the subjects of non-parametric interval estimation and progressive censoring, it becomes obvious that one of the main virtues of the non-parametric interval estimation is its distribution-free property and it makes the method applicable as a supplement to any continuous distribution families [9]. Certain drawbacks do exist, however. It may not be always possible to construct a confidence interval with a desired coverage level, particularly with a 
small sample size, and even if one can, the actual level of confidence will be separated in the neighborhood of the nominal level. With no exception, this intrinsic problem also occurs in the complete sample case (i.e., no censoring). One should therefore carefully select the values of $n, m$, and $\alpha$ in the designing stage so that they will certainly lead to find the corresponding optimal censoring scheme or a nearly optimal censoring scheme for a quantile of interest. For the future research, it is desired to develop and investigate several different types of non-parametric optimality criteria other than the expected interval mass considered in this project so that the properties of different objective functions and their best uses can be analyzed and compared. Another possibility of the future research is to conduct the optimization under several well-known parametric distributions (e.g., exponential, Weibull, log-normal, gamma, etc.) and to compare the results with the non-parametric results obtained in this project in order to assess how robust the non-parametric method can be. 


\section{Bibliography}

[1] Arnold, B. C., Balakrishnan, N., and Nagaraja, H. N. (1992), A First Course in Order Statistics, New York: John Wiley.

[2] Ashkar, F. and Ouarda, T. B. M. J. (1998), "Approximate confidence intervals for quantiles of gamma and generalized gamma distributions," Journal of Hydrologic Engineering, vol. 3, 43-51.

[3] Balakrishnan, N. and Aggarwala, R. A. (2000), Progressive Censoring: Theory, Methods, and Applications, Boston: Birkhäuser.

[4] Balakrishnan, N., Childs, A., and Chandrasekar, B. (2002), "An efficient computational method for moments of order statistics under progressive censoring," Statistics and Probability Letters, vol. 60, 359-365.

[5] Balakrishnan, N. and Cohen, A. C. (1991), Order Statistics and Inference: Estimation Methods, San Diego: Academic Press.

[6] Cohen, A. C. (1963), "Progressively censored samples in life testing," Technometrics, vol. 5, 327-329.

[7] David, H. A. (1981), Order Statistics, 2nd ed., New York: John Wiley. 
[8] Fligner, M. A. and Douglas, A. W. (1979), "Non-parametric prediction intervals for a future sample median," Journal of the American Statistical Association, vol. $74,453-456$.

[9] Gibbons, J. D. and Chakraborti, S. (1992), Nonparametric Statistical Inference, 3rd ed., New York: Dekker.

[10] Gouno, E., Sen, A., and Balakrishnan, N. (2004), "Optimal step-stress test under progressive Type-I censoring," IEEE Transactions on Reliability, vol. 53, 383393.

[11] Guilbaud, O. (2001), "Exact non-parametric confidence intervals for quantiles with progressive Type-II censoring," Scandinavian Journal of Statistics, vol. 28, 699-713.

[12] Guilbaud, O. (2004), "Exact non-parametric confidence, prediction and tolerance intervals with progressive Type-II censoring," Scandinavian Journal of Statistics, vol. $31,265-281$.

[13] Ng, H. K. T., Chan, P. S., and Balakrishnan, N. (2002), "Estimation of parameters from progressively censored data using EM algorithm," Computational Statistics and Data Analysis, vol. 39, 371-386.

[14] Viveros, R. and Balakrishnan, N. (1994), "Interval estimation of parameters of life from progressively censored data," Technometrics, vol. 36, 84-91. 


\section{Appendix A}

\section{Results of Numerical Study}

The optimization results of the numerical studies conducted in Section 5.2 are tabulated in this section. 
Table A.1: Optimal PC Schemes for Non-Parametric CIs of $\xi_{0.45}$ with $n=10$ and $m=7$ (total 84 progressive Type-II censoring schemes examined)

\begin{tabular}{|c|c|c|c|c|c|c|c|}
\hline$\alpha$ & Count & Category & Censoring Scheme (R) & $\mathrm{CI}^{2}$ & Actual $C L^{3}$ & CI Mass & Efficiency \\
\hline \multirow{3}{*}{0.01} & \multirow{3}{*}{2} & Best $\mathrm{PC}^{4}$ & $(0,3,0 \star 5)$ & {$[1,7]$} & 0.9918 & 0.7727 & $100.00 \%$ \\
\hline & & Worst PC & $(3,0 \star 6)$ & {$[1,7]$} & 0.9929 & 0.7792 & $99.17 \%$ \\
\hline & & Type-II & $(0 \star 6,3)$ & {$[1,7]$} & 0.8955 & 0.5455 & $\mathrm{NA}^{5}$ \\
\hline \multirow{3}{*}{0.05} & \multirow{3}{*}{74} & Best PC & $(0,2,0 \star 4,1)$ & {$[2,7]$} & 0.9536 & 0.5844 & $100.00 \%$ \\
\hline & & Worst PC & $(2,1,0 \star 5)$ & {$[1,7]$} & 0.9887 & 0.7765 & $75.26 \%$ \\
\hline & & Type-II & $(0 \star 6,3)$ & {$[1,7]$} & 0.8955 & 0.5455 & NA \\
\hline \multirow{3}{*}{0.10} & \multirow{3}{*}{83} & Best PC & $(0 \star 4,1,0,2)$ & {$[2,7]$} & 0.9046 & 0.4909 & $100.00 \%$ \\
\hline & & Worst PC & $(0,0,1,2,0 \star 3)$ & {$[2,7]$} & 0.9601 & 0.6623 & $74.12 \%$ \\
\hline & & Type-II & $(0 \star 6,3)$ & {$[1,7]$} & 0.8955 & 0.5455 & NA \\
\hline
\end{tabular}

\footnotetext{
2 confidence interval

3 confidence level

${ }^{4}$ progressive censoring

5 not available
} 
Table A.2: Optimal PC Schemes for Non-Parametric CIs of $\xi_{0.35}$ with $n=15$ and $m=6$ (total 2002 progressive Type-II censoring schemes examined)

\begin{tabular}{|c|c|c|c|c|c|c|c|}
\hline$\alpha$ & Count & Category & Censoring Scheme (R) & CI & Actual CL & CI Mass & Efficiency \\
\hline \hline \multirow{4}{*}{0.01} & & Best PC & $(0,9,0 \star 4)$ & {$[1,6]$} & 0.9928 & 0.7625 & $100.00 \%$ \\
& 2 & Worst PC & $(9,0 \star 5)$ & {$[1,6]$} & 0.9953 & 0.7812 & $97.60 \%$ \\
\cline { 3 - 8 } & & Type-II & $(0 \star 5,9)$ & {$[1,6]$} & 0.5627 & 0.3125 & NA \\
\hline \multirow{3}{*}{0.05} & \multirow{3}{*}{111} & Best PC & $(0,8,0 \star 3,1)$ & {$[2,6]$} & 0.9643 & 0.5833 & $100.00 \%$ \\
& & Worst PC & $(8,1,0 \star 4)$ & {$[1,6]$} & 0.9888 & 0.7768 & $75.10 \%$ \\
\cline { 3 - 8 } & & Type-II & $(0 \star 5,9)$ & {$[1,6]$} & 0.5627 & 0.3125 & NA \\
\hline \multirow{3}{*}{0.10} & \multirow{3}{*}{650} & Best PC & $(0,6,0,0,1,2)$ & {$[2,6]$} & 0.9036 & 0.4648 & $100.00 \%$ \\
& & Worst PC & $(7,1,1,0 \star 3)$ & {$[1,6]$} & 0.9785 & 0.7666 & $60.64 \%$ \\
\cline { 3 - 8 } & & Type-II & $(0 \star 5,9)$ & {$[1,6]$} & 0.5627 & 0.3125 & NA \\
\hline
\end{tabular}

Table A.3: Optimal PC Schemes for Non-Parametric CIs of $\xi_{0.35}$ with $n=15$ and $m=10$ (total 2002 progressive Type-II censoring schemes examined)

\begin{tabular}{|c|c|c|c|c|c|c|c|}
\hline$\alpha$ & Count & Category & Censoring Scheme (R) & CI & Actual CL & CI Mass & Efficiency \\
\hline \hline \multirow{3}{*}{0.01} & \multirow{3}{*}{1997} & Best PC & $(0 \star 5,1,0 \star 3,4)$ & {$[1,10]$} & 0.9903 & 0.5903 & $100.00 \%$ \\
& & Worst PC & $(0,0,1,0,1,1,2,0 \star 3)$ & {$[1,10]$} & 0.9974 & 0.8085 & $73.01 \%$ \\
\cline { 3 - 8 } & & Type-II & $(0 \star 9,5)$ & {$[1,10]$} & 0.9860 & 0.5625 & NA \\
\hline \multirow{3}{*}{0.05} & \multirow{3}{*}{2002} & Best PC & $(0 \star 6,1,0,0,4)$ & {$[2,9]$} & 0.9511 & 0.4531 & $100.00 \%$ \\
& & Worst PC & $(4,0 \star 5,1,0 \star 3)$ & {$[1,8]$} & 0.9855 & 0.6179 & $73.33 \%$ \\
\cline { 3 - 8 } & & Type-II & $(0 \star 9,5)$ & {$[2,10]$} & 0.9734 & 0.5000 & $90.62 \%$ \\
\hline \multirow{3}{*}{0.10} & \multirow{3}{*}{2002} & Best PC & $(0 \star 7,1,0,4)$ & {$[3,9]$} & 0.9003 & 0.3839 & $100.00 \%$ \\
& & Worst PC & $(0,2,0,0,2,1,0 \star 4)$ & {$[2,8]$} & 0.9602 & 0.5375 & $71.43 \%$ \\
\cline { 3 - 8 } & & Type-II & $(0 \star 9,5)$ & {$[3,10]$} & 0.9258 & 0.4375 & $87.76 \%$ \\
\hline
\end{tabular}


Table A.4: Optimal PC Schemes for Non-Parametric CIs of $\xi_{0.50}$ with $n=15$ and $m=10$ (total 2002 progressive Type-II censoring schemes examined)

\begin{tabular}{|c|c|c|c|c|c|c|c|}
\hline$\alpha$ & Count & Category & Censoring Scheme (R) & CI & Actual CL & CI Mass & Efficiency \\
\hline \hline \multirow{3}{*}{0.01} & \multirow{3}{*}{212} & Best PC & $(4,0 \star 8,1)$ & {$[2,10]$} & 0.9904 & 0.6818 & $100.00 \%$ \\
& & Worst PC & $(1 \star 5,0 \star 5)$ & {$[1,10]$} & 0.9901 & 0.8328 & $81.87 \%$ \\
\cline { 3 - 8 } & & Type-II & $(0 \star 9,5)$ & {$[1,10]$} & 0.8491 & 0.5625 & NA \\
\hline \multirow{3}{*}{0.05} & \multirow{3}{*}{1831} & Best PC & $(0 \star 3,3,0 \star 5,2)$ & {$[4,10]$} & 0.9524 & 0.5000 & $100.00 \%$ \\
& & Worst PC & $(0 \star 4,1,0,0,1,3,0)$ & {$[1,10]$} & 0.9501 & 0.7370 & $67.84 \%$ \\
\cline { 3 - 8 } & & Type-II & $(0 \star 9,5)$ & {$[1,10]$} & 0.8491 & 0.5625 & NA \\
\hline \multirow{3}{*}{0.10} & \multirow{3}{*}{1992} & Best PC & $(0,0,1,0 \star 4,1,0,3)$ & {$[4,10]$} & 0.9004 & 0.4288 & $100.00 \%$ \\
& & Worst PC & $(0 \star 3,2,0,0,2,1,0,0)$ & {$[4,10]$} & 0.9609 & 0.6100 & $70.30 \%$ \\
\cline { 3 - 8 } & & Type-II & $(0 \star 9,5)$ & {$[1,10]$} & 0.8491 & 0.5625 & NA \\
\hline
\end{tabular}

Table A.5: Optimal PC Schemes for Non-Parametric CIs of $\xi_{0.25}$ with $n=20$ and $m=6$ (total 11628 progressive Type-II censoring schemes examined)

\begin{tabular}{|c|c|c|c|c|c|c|c|}
\hline$\alpha$ & Count & Category & Censoring Scheme (R) & CI & Actual CL & CI Mass & Efficiency \\
\hline \hline \multirow{3}{*}{0.01} & \multirow{3}{*}{26} & Best PC & $(0,13,0 \star 3,1)$ & {$[1,6]$} & 0.9915 & 0.6508 & $100.00 \%$ \\
& & Worst PC & $(14,0 \star 5)$ & {$[1,6]$} & 0.9963 & 0.7937 & $82.00 \%$ \\
\cline { 3 - 8 } & & Type-II & $(0 \star 5,14)$ & {$[1,6]$} & 0.6140 & 0.2381 & NA \\
\hline \multirow{3}{*}{0.05} & \multirow{3}{*}{1302} & Best PC & $(9,0 \star 3,1,4)$ & {$[1,6]$} & 0.9523 & 0.4473 & $100.00 \%$ \\
& & Worst PC & $(10,4,0 \star 4)$ & {$[1,6]$} & 0.9853 & 0.7810 & $57.28 \%$ \\
\cline { 3 - 8 } & & Type-II & $(0 \star 5,14)$ & {$[1,6]$} & 0.6140 & 0.2381 & NA \\
\hline \multirow{3}{*}{0.10} & \multirow{3}{*}{5888} & Best PC & $(0,9,0 \star 3,5)$ & {$[2,6]$} & 0.9097 & 0.3619 & $100.00 \%$ \\
& & Worst PC & $(7,5,2,0 \star 3)$ & {$[1,6]$} & 0.9711 & 0.7640 & $47.37 \%$ \\
\cline { 3 - 8 } & & Type-II & $(0 \star 5,14)$ & {$[1,6]$} & 0.6140 & 0.2381 & NA \\
\hline
\end{tabular}


Table A.6: Optimal PC Schemes for Non-Parametric CIs of $\xi_{0.25}$ with $n=20$ and $m=10$ (total 92378 progressive Type-II censoring schemes examined)

\begin{tabular}{|c|c|c|c|c|c|c|c|}
\hline$\alpha$ & Count & Category & Censoring Scheme (R) & CI & Actual CL & CI Mass & Efficiency \\
\hline \hline \multirow{3}{*}{0.01} & \multirow{3}{*}{92207} & Best PC & $(0,1,0,1,0 \star 5,8)$ & {$[1,10]$} & 0.9900 & 0.4698 & $100.00 \%$ \\
& & Worst PC & $(0 \star 4,1,3,6,0 \star 3)$ & {$[1,10]$} & 0.9960 & 0.7908 & $59.42 \%$ \\
\cline { 3 - 8 } & & Type-II & $(0 \star 9,10)$ & {$[1,10]$} & 0.9830 & 0.4286 & NA \\
\hline \multirow{3}{*}{0.05} & \multirow{3}{*}{92378} & Best PC & $(0,0,1,0,1,0 \star 4,8)$ & {$[2,9]$} & 0.9501 & 0.3645 & $100.00 \%$ \\
& & Worst PC & $(4,0,0,1,4,1,0 \star 4)$ & {$[1,8]$} & 0.9865 & 0.5876 & $62.04 \%$ \\
\cline { 3 - 8 } & & Type-II & $(0 \star 9,10)$ & {$[2,10]$} & 0.9618 & 0.3810 & $95.69 \%$ \\
\hline \multirow{3}{*}{0.10} & \multirow{3}{*}{92378} & Best PC & $(0,0,1,0 \star 3,2,0,0,7)$ & {$[2,8]$} & 0.9001 & 0.3089 & $100.00 \%$ \\
& & Worst PC & $(0 \star 6,1,9,0,0)$ & {$[2,9]$} & 0.9635 & 0.4945 & $62.46 \%$ \\
\cline { 3 - 8 } & & Type-II & $(0 \star 9,10)$ & {$[2,9]$} & 0.9348 & 0.3333 & $92.67 \%$ \\
\hline
\end{tabular}

Table A.7: Optimal PC Schemes for Non-Parametric CIs of $\xi_{0.35}$ with $n=20$ and $m=10$ (total 92378 progressive Type-II censoring schemes examined)

\begin{tabular}{|c|c|c|c|c|c|c|c|}
\hline$\alpha$ & Count & Category & Censoring Scheme (R) & CI & Actual CL & CI Mass & Efficiency \\
\hline \hline \multirow{3}{*}{0.01} & \multirow{3}{*}{36115} & Best PC & $(0,6,0 \star 5,1,0,3)$ & {$[2,10]$} & 0.9903 & 0.5800 & $100.00 \%$ \\
& & Worst PC & $(6,0,0,2,2,0 \star 5)$ & {$[1,10]$} & 0.9982 & 0.8415 & $68.92 \%$ \\
\cline { 3 - 8 } & & Type-II & $(0 \star 9,10)$ & {$[1,10]$} & 0.8780 & 0.4286 & NA \\
\hline \multirow{3}{*}{0.05} & \multirow{3}{*}{91386} & Best PC & $(0 \star 3,4,0 \star 5,6)$ & {$[3,10]$} & 0.9514 & 0.4212 & $100.00 \%$ \\
& & Worst PC & $(0 \star 3,2,2,2,4,0 \star 3)$ & {$[3,10]$} & 0.9773 & 0.7032 & $59.90 \%$ \\
\cline { 3 - 8 } & & Type-II & $(0 \star 9,10)$ & {$[1,10]$} & 0.8780 & 0.4286 & NA \\
\hline \multirow{3}{*}{0.10} & \multirow{3}{*}{92369} & Best PC & $(0 \star 3,3,0 \star 5,7)$ & {$[4,10]$} & 0.9025 & 0.3469 & $100.00 \%$ \\
& & Worst PC & $(1,2,0 \star 5,2,5,0)$ & {$[3,10]$} & 0.9572 & 0.5992 & $57.90 \%$ \\
\cline { 3 - 8 } & & Type-II & $(0 \star 9,10)$ & {$[1,10]$} & 0.8780 & 0.4286 & NA \\
\hline
\end{tabular}


Table A.8: Optimal PC Schemes for Non-Parametric CIs of $\xi_{0.50}$ with $n=20$ and $m=10$ (total 92378 progressive Type-II censoring schemes examined)

\begin{tabular}{|c|c|c|c|c|c|c|c|}
\hline$\alpha$ & Count & Category & Censoring Scheme (R) & CI & Actual CL & CI Mass & Efficiency \\
\hline \hline \multirow{4}{*}{0.01} & \multirow{3}{*}{32} & Best PC & $(0 \star 4,10,0 \star 5)$ & {$[4,10]$} & 0.9922 & 0.6825 & $100.00 \%$ \\
& & Worst PC & $(8,1,1,0 \star 7)$ & {$[1,10]$} & 0.9905 & 0.8542 & $79.91 \%$ \\
\cline { 3 - 8 } & & Type-II & $(0 \star 9,10)$ & {$[1,10]$} & 0.4119 & 0.4286 & NA \\
\hline \multirow{4}{*}{0.05} & \multirow{3}{*}{3550} & Best PC & $(0,0,8,0 \star 6,2)$ & {$[4,10]$} & 0.9507 & 0.5143 & $100.00 \%$ \\
& & Worst PC & $(4,1,1,2,1,0,1,0 \star 3)$ & {$[1,10]$} & 0.9500 & 0.8317 & $61.83 \%$ \\
\cline { 3 - 8 } & & Type-II & $(0 \star 9,10)$ & {$[1,10]$} & 0.4119 & 0.4286 & NA \\
\hline \multirow{3}{*}{0.10} & \multirow{2}{*}{27873} & Best PC & $(7,0 \star 8,3)$ & {$[4,10]$} & 0.9143 & 0.4396 & $100.00 \%$ \\
& & Worst PC & $(0,1,0,3,1,1,4,0 \star 3)$ & {$[1,10]$} & 0.9000 & 0.8024 & $54.78 \%$ \\
\cline { 3 - 8 } & & Type-II & $(0 \star 9,10)$ & {$[1,10]$} & 0.4119 & 0.4286 & NA \\
\hline
\end{tabular}

Table A.9: Optimal PC Schemes for Non-Parametric CIs of $\xi_{0.25}$ with $n=20$ and $m=16$ (total 3876 progressive Type-II censoring schemes examined)

\begin{tabular}{|c|c|c|c|c|c|c|r|}
\hline$\alpha$ & Count & Category & Censoring Scheme (R) & CI & Actual CL & CI Mass & Efficiency \\
\hline \hline \multirow{3}{*}{0.01} & \multirow{3}{*}{3876} & Best PC & $(0,1,0,1,0 \star 11,2)$ & {$[1,10]$} & 0.9900 & 0.4698 & $100.00 \%$ \\
& & Worst PC & $(0 \star 5,1,1,2,0 \star 8)$ & {$[1,11]$} & 0.9955 & 0.5471 & $85.89 \%$ \\
\cline { 3 - 8 } & Type-II & $(0 \star 15,4)$ & {$[1,11]$} & 0.9929 & 0.4762 & $98.67 \%$ \\
\hline \multirow{4}{*}{0.05} & \multirow{3}{*}{3876} & Best PC & $(0,0,1,0,1,0 \star 10,2)$ & {$[2,9]$} & 0.9501 & 0.3645 & $100.00 \%$ \\
& & Worst PC & $(2,0 \star 6,2,0 \star 8)$ & {$[1,9]$} & 0.9789 & 0.4350 & $83.80 \%$ \\
\cline { 3 - 8 } & & Type-II & $(0 \star 15,4)$ & {$[2,10]$} & 0.9618 & 0.3810 & $95.69 \%$ \\
\hline \multirow{3}{*}{0.10} & \multirow{3}{*}{3876} & Best PC & $(0,0,1,0 \star 3,2,0 \star 8,1)$ & {$[2,8]$} & 0.9001 & 0.3089 & $100.00 \%$ \\
& & Worst PC & $(0,4,0 \star 14)$ & {$[2,8]$} & 0.9410 & 0.3619 & $85.35 \%$ \\
\cline { 3 - 8 } & & Type-II & $(0 \star 15,4)$ & {$[2,9]$} & 0.9348 & 0.3333 & $92.67 \%$ \\
\hline
\end{tabular}


Table A.10: Optimal PC Schemes for Non-Parametric CIs of $\xi_{0.35}$ with $n=20$ and $m=16$ (total 3876 progressive Type-II censoring schemes examined)

\begin{tabular}{|c|c|c|c|c|c|c|c|}
\hline$\alpha$ & Count & Category & Censoring Scheme (R) & CI & Actual CL & CI Mass & Efficiency \\
\hline \hline \multirow{3}{*}{0.01} & \multirow{3}{*}{3876} & Best PC & $(0 \star 15,4)$ & {$[2,13]$} & 0.9919 & 0.5238 & $100.00 \%$ \\
& & Worst PC & $(0 \star 7,1,1,0,2,0 \star 5)$ & {$[2,13]$} & 0.9959 & 0.6021 & $86.99 \%$ \\
\cline { 2 - 8 } & & Type-II & $(0 \star 15,4)$ & {$[2,13]$} & 0.9919 & 0.5238 & $100.00 \%$ \\
\hline \multirow{3}{*}{0.05} & \multirow{3}{*}{3876} & Best PC & $(0,0,1,0 \star 12,3)$ & {$[3,11]$} & 0.9505 & 0.4034 & $100.00 \%$ \\
& & Worst PC & $(0,4,0 \star 14)$ & {$[3,11]$} & 0.9693 & 0.4825 & $83.59 \%$ \\
\cline { 3 - 9 } & & Type-II & $(0 \star 15,4)$ & {$[3,12]$} & 0.9683 & 0.4286 & $94.12 \%$ \\
\hline \multirow{3}{*}{0.10} & \multirow{3}{*}{3876} & Best PC & $(0 \star 15,4)$ & {$[4,11]$} & 0.9025 & 0.3333 & $100.00 \%$ \\
& & Worst PC & $(0,1,2,1,0 \star 12)$ & {$[3,10]$} & 0.9491 & 0.4251 & $78.42 \%$ \\
\cline { 3 - 8 } & & Type-II & $(0 \star 15,4)$ & {$[4,11]$} & 0.9025 & 0.3333 & $100.00 \%$ \\
\hline
\end{tabular}

Table A.11: Optimal PC Schemes for Non-Parametric CIs of $\xi_{0.50}$ with $n=20$ and $m=16$ (total 3876 progressive Type-II censoring schemes examined)

\begin{tabular}{|c|c|c|c|c|c|c|c|}
\hline$\alpha$ & Count & Category & Censoring Scheme (R) & CI & Actual CL & CI Mass & Efficiency \\
\hline \hline \multirow{4}{*}{0.01} & \multirow{3}{*}{3876} & Best PC & $(0 \star 12,1,0,0,3)$ & {$[5,16]$} & 0.9902 & 0.5442 & $100.00 \%$ \\
& & Worst PC & $(0,0,1,1,0 \star 5,1,1,0 \star 5)$ & {$[4,15]$} & 0.9967 & 0.6655 & $81.77 \%$ \\
\cline { 2 - 9 } & & Type-II & $(0 \star 15,4)$ & {$[4,16]$} & 0.9928 & 0.5714 & $95.24 \%$ \\
\hline \multirow{3}{*}{0.05} & \multirow{3}{*}{3876} & Best PC & $(0 \star 6,1,0 \star 3,1,0 \star 4,2)$ & {$[6,14]$} & 0.9503 & 0.4258 & $100.00 \%$ \\
& & Worst PC & $(0,1,1,0 \star 8,1,1,0 \star 3)$ & {$[5,14]$} & 0.9761 & 0.5140 & $82.84 \%$ \\
\cline { 3 - 9 } & & Type-II & $(0 \star 15,4)$ & {$[6,15]$} & 0.9586 & 0.4286 & $99.36 \%$ \\
\hline \multirow{3}{*}{0.10} & \multirow{3}{*}{3876} & Best PC & $(0 \star 8,1,0 \star 6,3)$ & {$[7,14]$} & 0.9015 & 0.3550 & $100.00 \%$ \\
& & Worst PC & $(0 \star 5,2,0,2,0 \star 8)$ & {$[6,13]$} & 0.9484 & 0.4457 & $79.65 \%$ \\
\cline { 3 - 9 } & & Type-II & $(0 \star 15,4)$ & {$[6,14]$} & 0.9216 & 0.3810 & $93.18 \%$ \\
\hline
\end{tabular}


Table A.12: Optimal PC Schemes for Non-Parametric CIs of $\xi_{0.65}$ with $n=20$ and $m=16$ (total 3876 progressive Type-II censoring schemes examined)

\begin{tabular}{|c|c|c|c|c|c|c|c|}
\hline$\alpha$ & Count & Category & Censoring Scheme (R) & CI & Actual CL & CI Mass & Efficiency \\
\hline \hline \multirow{3}{*}{0.01} & \multirow{3}{*}{1614} & Best PC & $(0 \star 9,4,0 \star 6)$ & {$[8,16]$} & 0.9903 & 0.5442 & $100.00 \%$ \\
& & Worst PC & $(0 \star 6,2,0 \star 3,1,1,0 \star 4)$ & {$[3,16]$} & 0.9900 & 0.7810 & $69.69 \%$ \\
\cline { 3 - 8 } & & Type-II & $(0 \star 15,4)$ & {$[1,16]$} & 0.8818 & 0.7143 & NA \\
\hline \multirow{4}{*}{0.05} & \multirow{3}{*}{3823} & Best PC & $(0 \star 12,3,0,0,1)$ & {$[9,16]$} & 0.9507 & 0.4190 & $100.00 \%$ \\
& & Worst PC & $(0 \star 10,1,0,0,1 \star 3)$ & {$[4,16]$} & 0.9500 & 0.6402 & $65.45 \%$ \\
\cline { 3 - 8 } & & Type-II & $(0 \star 15,4)$ & {$[1,16]$} & 0.8818 & 0.7143 & NA \\
\hline \multirow{3}{*}{0.10} & \multirow{3}{*}{3874} & Best PC & $(0 \star 10,2,0 \star 4,2)$ & {$[10,16]$} & 0.9036 & 0.3452 & $100.00 \%$ \\
& & Worst PC & $(0,0,1,0 \star 8,1,0,2,0,0)$ & {$[9,16]$} & 0.9489 & 0.4586 & $75.28 \%$ \\
\cline { 3 - 8 } & & Type-II & $(0 \star 15,4)$ & {$[1,16]$} & 0.8818 & 0.7143 & NA \\
\hline
\end{tabular}


Table A.13: Optimal PC Schemes for Non-Parametric CIs of $\xi_{0.25}$ with $n=25$ and $m=5$ (total 10626 progressive Type-II censoring schemes examined)

\begin{tabular}{|c|c|c|c|c|c|c|c|}
\hline$\alpha$ & Count & Category & Censoring Scheme (R) & CI & Actual CL & CI Mass & Efficiency \\
\hline \hline \multirow{4}{*}{0.01} & \multirow{3}{*}{3} & Best PC & $(0,20,0 \star 3)$ & {$[1,5]$} & 0.9918 & 0.7308 & $100.00 \%$ \\
& \multirow{4}{*}{0.05} & Worst PC & $(20,0 \star 4)$ & {$[1,5]$} & 0.9967 & 0.7692 & $95.00 \%$ \\
\cline { 3 - 8 } & & Type-II & $(0 \star 4,20)$ & {$[1,5]$} & 0.2130 & 0.1538 & NA \\
\hline \multirow{4}{*}{0.10} & \multirow{4}{*}{49} & Best PC & $(17,0 \star 3,3)$ & {$[1,5]$} & 0.9507 & 0.4808 & $100.00 \%$ \\
& \multirow{3}{*}{177} & Worst PC & $(19,1,0 \star 3)$ & {$[1,5]$} & 0.9902 & 0.7612 & $63.16 \%$ \\
\cline { 3 - 8 } & & Type-II & $(0 \star 4,20)$ & {$[1,5]$} & 0.2130 & 0.1538 & NA \\
\cline { 3 - 8 } & & Best PC & $(0,17,0,0,3)$ & {$[2,5]$} & 0.9028 & 0.3956 & $100.00 \%$ \\
\cline { 3 - 8 } & & Worst PC & $(18,2,0 \star 3)$ & {$[1,5]$} & 0.9795 & 0.7555 & $52.36 \%$ \\
\cline { 3 - 8 } & & Type-II & $(0 \star 4,20)$ & {$[1,5]$} & 0.2130 & 0.1538 & NA \\
\hline
\end{tabular}

Table A.14: Optimal PC Schemes for Non-Parametric Cls of $\xi_{0.25}$ with $n=25$ and $m=10$ (total 1307504 progressive Type-II censoring schemes examined)

\begin{tabular}{|c|c|c|c|c|c|c|c|}
\hline$\alpha$ & Count & Category & Censoring Scheme (R) & CI & Actual CL & CI Mass & Efficiency \\
\hline \hline \multirow{3}{*}{0.01} & \multirow{3}{*}{1061107} & Best PC & $(6,0 \star 5,1,0,0,8)$ & {$[1,10]$} & 0.9902 & 0.4681 & $100.00 \%$ \\
& & Worst PC & $(2,1,3,4,3,2,0 \star 4)$ & {$[1,10]$} & 0.9975 & 0.8293 & $56.45 \%$ \\
\cline { 3 - 9 } & & Type-II & $(0 \star 9,15)$ & {$[1,10]$} & 0.9279 & 0.3462 & NA \\
\hline \multirow{3}{*}{0.05} & \multirow{3}{*}{1307377} & Best PC & $(0,2,0 \star 6,1,12)$ & {$[2,10]$} & 0.9505 & 0.3387 & $100.00 \%$ \\
& & Worst PC & $(0 \star 4,1,2,5,7,0,0)$ & {$[3,10]$} & 0.9584 & 0.6658 & $50.87 \%$ \\
\cline { 3 - 9 } & & Type-II & $(0 \star 9,15)$ & {$[1,10]$} & 0.9279 & 0.3462 & NA \\
\hline \multirow{3}{*}{0.10} & \multirow{2}{*}{1307504} & Best PC & $(0 \star 7,1,0,14)$ & {$[3,10]$} & 0.9015 & 0.2738 & $100.00 \%$ \\
& & Worst PC & $(0 \star 6,2,6,7,0)$ & {$[3,10]$} & 0.9479 & 0.5751 & $47.60 \%$ \\
\cline { 3 - 9 } & & Type-II & $(0 \star 9,15)$ & {$[2,10]$} & 0.9216 & 0.3077 & $88.97 \%$ \\
\hline
\end{tabular}


Table A.15: Optimal PC Schemes for Non-Parametric CIs of $\xi_{0.35}$ with $n=25$ and $m=10$ (total 1307504 progressive Type-II censoring schemes examined)

\begin{tabular}{|c|c|c|c|c|c|c|c|}
\hline$\alpha$ & Count & Category & Censoring Scheme (R) & CI & Actual CL & CI Mass & Efficiency \\
\hline \hline \multirow{4}{*}{0.01} & \multirow{3}{*}{11179} & Best PC & $(0,0,12,0 \star 6,3)$ & {$[3,10]$} & 0.9908 & 0.5629 & $100.00 \%$ \\
& & Worst PC & $(11,1,1,2,0 \star 6)$ & {$[1,10]$} & 0.9976 & 0.8563 & $65.74 \%$ \\
\cline { 3 - 9 } & & Type-II & $(0 \star 9,15)$ & {$[1,10]$} & 0.6303 & 0.3462 & NA \\
\hline \multirow{3}{*}{0.05} & \multirow{3}{*}{630981} & Best PC & $(0 \star 3,10,0 \star 5,5)$ & {$[4,10]$} & 0.9557 & 0.4231 & $100.00 \%$ \\
& & Worst PC & $(0,1,0,1,3,4,5,1,0,0)$ & {$[1,10]$} & 0.9502 & 0.7796 & $54.27 \%$ \\
\cline { 3 - 9 } & & Type-II & $(0 \star 9,15)$ & {$[1,10]$} & 0.6303 & 0.3462 & NA \\
\hline \multirow{3}{*}{0.10} & \multirow{3}{*}{1165349} & Best PC & $(0 \star 5,10,0 \star 3,5)$ & {$[5,10]$} & 0.9020 & 0.3462 & $100.00 \%$ \\
& & Worst PC & $(0 \star 5,2,4,7,2,0)$ & {$[1,10]$} & 0.9000 & 0.6933 & $49.93 \%$ \\
\cline { 3 - 9 } & & Type-II & $(0 \star 9,15)$ & {$[1,10]$} & 0.6303 & 0.3462 & NA \\
\hline
\end{tabular}

Table A.16: Optimal PC Schemes for Non-Parametric CIs of $\xi_{0.50}$ with $n=25$ and $m=10$ (total 1307504 progressive Type-II censoring schemes examined)

\begin{tabular}{|c|c|c|c|c|c|c|c|}
\hline$\alpha$ & Count & Category & Censoring Scheme (R) & CI & Actual CL & CI Mass & Efficiency \\
\hline \multirow{3}{*}{0.01} & \multirow{3}{*}{21} & Best PC & $(0 \star 4,15,0 \star 5)$ & {$[5,10]$} & 0.9903 & 0.6731 & $100.00 \%$ \\
\hline & & Worst PC & $(0,15,0 \star 8)$ & {$[2,10]$} & 0.9979 & 0.8205 & $82.03 \%$ \\
\hline & & Type-II & $(0 \star 9,15)$ & {$[1,10]$} & 0.1148 & 0.3462 & NA \\
\hline \multirow{3}{*}{0.05} & \multirow{3}{*}{766} & Best PC & $(0 \star 6,15,0 \star 3)$ & {$[7,10]$} & 0.9577 & 0.5481 & $100.00 \%$ \\
\hline & & Worst PC & $(11,0,1 \star 3,0,0,1,0,0)$ & {$[1,10]$} & 0.9502 & 0.8347 & $65.66 \%$ \\
\hline & & Type-II & $(0 \star 9,15)$ & {$[1,10]$} & 0.1148 & 0.3462 & NA \\
\hline \multirow{3}{*}{0.10} & \multirow{3}{*}{7122} & Best PC & $(0,0,13,0 \star 6,2)$ & {$[5,10]$} & 0.9016 & 0.4423 & $100.00 \%$ \\
\hline & & Worst PC & $(8,0,3,2,0,2,0 \star 4)$ & {$[1,10]$} & 0.9000 & 0.8406 & $52.62 \%$ \\
\hline & & Type-II & $(0 \star 9,15)$ & {$[1,10]$} & 0.1148 & 0.3462 & NA \\
\hline
\end{tabular}


Table A.17: Optimal PC Schemes for Non-Parametric CIs of $\xi_{0.25}$ with $n=25$ and $m=15$ (total 1961256 progressive Type-II censoring schemes examined)

\begin{tabular}{|c|c|c|c|c|c|c|r|}
\hline$\alpha$ & Count & Category & Censoring Scheme (R) & CI & Actual CL & CI Mass & Efficiency \\
\hline \hline \multirow{4}{*}{0.01} & \multirow{3}{*}{1961256} & Best PC & $(0 \star 9,1,0 \star 4,9)$ & {$[2,13]$} & 0.9901 & 0.4308 & $100.00 \%$ \\
& & Worst PC & $(1,0,1,2,3,3,0 \star 9)$ & {$[1,11]$} & 0.9972 & 0.5935 & $72.59 \%$ \\
\cline { 3 - 8 } & & Type-II & $(0 \star 14,10)$ & {$[2,14]$} & 0.9921 & 0.4615 & $93.33 \%$ \\
\hline \multirow{4}{*}{0.05} & \multirow{3}{*}{1961256} & Best PC & $(0,0,1,1,0 \star 10,8)$ & {$[3,11]$} & 0.9501 & 0.3358 & $100.00 \%$ \\
& & Worst PC & $(0,6,4,0 \star 12)$ & {$[2,9]$} & 0.9781 & 0.4536 & $74.01 \%$ \\
\cline { 3 - 8 } & & Type-II & $(0 \star 14,10)$ & {$[3,12]$} & 0.9572 & 0.3462 & $96.99 \%$ \\
\hline \multirow{3}{*}{0.10} & \multirow{2}{*}{1961256} & Best PC & $(0 \star 7,1,0 \star 6,9)$ & {$[3,10]$} & 0.9015 & 0.2738 & $100.00 \%$ \\
& & Worst PC & $(1,9,0 \star 13)$ & {$[2,8]$} & 0.9577 & 0.3949 & $69.32 \%$ \\
\cline { 3 - 8 } & & Type-II & $(0 \star 14,10)$ & {$[3,11]$} & 0.9382 & 0.3077 & $88.97 \%$ \\
\hline
\end{tabular}

Table A.18: Optimal PC Schemes for Non-Parametric CIs of $\xi_{0.35}$ with $n=25$ and $m=15$ (total 1961256 progressive Type-II censoring schemes examined)

\begin{tabular}{|c|c|c|c|c|c|c|c|}
\hline$\alpha$ & Count & Category & Censoring Scheme (R) & CI & Actual CL & CI Mass & Efficiency \\
\hline \hline \multirow{3}{*}{0.01} & \multirow{3}{*}{1961256} & Best PC & $(0 \star 11,1,0,0,9)$ & {$[3,15]$} & 0.9900 & 0.4704 & $100.00 \%$ \\
& & Worst PC & $(0 \star 10,1,3,5,1,0)$ & {$[3,15]$} & 0.9964 & 0.7038 & $66.84 \%$ \\
\cline { 3 - 8 } & & Type-II & $(0 \star 14,10)$ & {$[2,15]$} & 0.9904 & 0.5000 & $94.08 \%$ \\
\hline \multirow{4}{*}{0.05} & \multirow{3}{*}{1961256} & Best PC & $(0 \star 5,1,0 \star 6,1,0,8)$ & {$[5,14]$} & 0.9503 & 0.3660 & $100.00 \%$ \\
& & Worst PC & $(1,3,4,2,0 \star 11)$ & {$[3,11]$} & 0.9787 & 0.5350 & $68.42 \%$ \\
\cline { 3 - 8 } & & Type-II & $(0 \star 14,10)$ & {$[4,14]$} & 0.9649 & 0.3846 & $95.17 \%$ \\
\hline \multirow{3}{*}{0.10} & \multirow{2}{*}{1961256} & Best PC & $(0 \star 14,10)$ & {$[5,13]$} & 0.9075 & 0.3077 & $100.00 \%$ \\
& & Worst PC & $(4,3,3,0 \star 12)$ & {$[4,11]$} & 0.9286 & 0.4641 & $66.30 \%$ \\
\cline { 3 - 8 } & & Type-II & $(0 \star 14,10)$ & {$[5,13]$} & 0.9075 & 0.3077 & $100.00 \%$ \\
\hline
\end{tabular}


Table A.19: Optimal PC Schemes for Non-Parametric CIs of $\xi_{0.50}$ with $n=25$ and $m=15$ (total 1961256 progressive Type-II censoring schemes examined)

\begin{tabular}{|c|c|c|c|c|c|c|c|}
\hline$\alpha$ & Count & Category & Censoring Scheme (R) & CI & Actual CL & CI Mass & Efficiency \\
\hline \hline \multirow{3}{*}{0.01} & \multirow{3}{*}{571742} & West PC & $(0 \star 5,9,0 \star 8,1)$ & {$[6,14]$} & 0.9903 & 0.5594 & $100.00 \%$ \\
& & Worst PC & $(0,1,0,1,2,0,1 \star 3,2,0,1,0 \star 3)$ & {$[1,15]$} & 0.9900 & 0.8649 & $64.68 \%$ \\
\cline { 3 - 9 } & & Type-II & $(0 \star 14,10)$ & {$[1,15]$} & 0.7878 & 0.5385 & NA \\
\hline \multirow{3}{*}{0.05} & \multirow{3}{*}{1908500} & Best PC & $(0 \star 7,6,0 \star 6,4)$ & {$[8,15]$} & 0.9506 & 0.4038 & $100.00 \%$ \\
& & Worst PC & $(1,0 \star 7,1,0,1,0,4,3,0)$ & {$[1,15]$} & 0.9500 & 0.7686 & $52.54 \%$ \\
\cline { 3 - 9 } & & Type-II & $(0 \star 14,10)$ & {$[1,15]$} & 0.7878 & 0.5385 & NA \\
\hline \multirow{3}{*}{0.10} & \multirow{2}{*}{1959458} & Best PC & $(1,0 \star 6,3,0 \star 6,6)$ & {$[8,15]$} & 0.9010 & 0.3405 & $100.00 \%$ \\
& & Worst PC & $(0 \star 3,1,0 \star 6,1,1,0,3,4)$ & {$[2,15]$} & 0.9000 & 0.5680 & $59.95 \%$ \\
\cline { 3 - 9 } & & Type-II & $(0 \star 14,10)$ & {$[1,15]$} & 0.7878 & 0.5385 & NA \\
\hline
\end{tabular}

Table A.20: Optimal PC Schemes for Non-Parametric CIs of $\xi_{0.65}$ with $n=25$ and $m=15$ (total 1961256 progressive Type-II censoring schemes examined)

\begin{tabular}{|c|c|c|c|c|c|c|c|}
\hline$\alpha$ & Count & Category & Censoring Scheme (R) & CI & Actual CL & CI Mass & Efficiency \\
\hline \hline \multirow{4}{*}{0.01} & \multirow{3}{*}{43} & Best PC & $(0 \star 4,10,0 \star 10)$ & {$[7,15]$} & 0.9909 & 0.5874 & $100.00 \%$ \\
& & Worst PC & $(0 \star 3,9,1,0 \star 10)$ & {$[5,15]$} & 0.9905 & 0.7101 & $82.73 \%$ \\
\cline { 2 - 8 } & & Type-II & $(0 \star 14,10)$ & {$[1,15]$} & 0.2288 & 0.5385 & NA \\
\hline \multirow{3}{*}{0.05} & \multirow{3}{*}{14507} & Best PC & $(0,0,9,0 \star 11,1)$ & {$[8,15]$} & 0.9509 & 0.4423 & $100.00 \%$ \\
& & Worst PC & $(2,1,3,0,2,2,0 \star 9)$ & {$[3,15]$} & 0.9500 & 0.8046 & $54.97 \%$ \\
\cline { 3 - 9 } & & Type-II & $(0 \star 14,10)$ & {$[1,15]$} & 0.2288 & 0.5385 & NA \\
\hline \multirow{3}{*}{0.10} & \multirow{2}{*}{262889} & Best PC & $(0 \star 3,8,0 \star 10,2)$ & {$[9,15]$} & 0.9009 & 0.3626 & $100.00 \%$ \\
& & Worst PC & $(1 \star 3,0,0,4,0,0,1,0,0,2,0 \star 3)$ & {$[2,15]$} & 0.9000 & 0.8256 & $43.92 \%$ \\
\cline { 3 - 9 } & & Type-II & $(0 \star 14,10)$ & {$[1,15]$} & 0.2288 & 0.5385 & NA \\
\hline
\end{tabular}


Table A.21: Optimal PC Schemes for Non-Parametric CIs of $\xi_{0.25}$ with $n=25$ and $m=20$ (total 42504 progressive Type-II censoring schemes examined)

\begin{tabular}{|c|c|c|c|c|c|c|c|}
\hline$\alpha$ & Count & Category & Censoring Scheme (R) & CI & Actual CL & CI Mass & Efficiency \\
\hline \hline \multirow{3}{*}{0.01} & \multirow{3}{*}{42504} & Best PC & $(0 \star 9,1,0 \star 9,4)$ & {$[2,13]$} & 0.9901 & 0.4308 & $100.00 \%$ \\
& & Worst PC & $(1,0 \star 3,2,1,1,0 \star 13)$ & {$[1,12]$} & 0.9968 & 0.5055 & $85.22 \%$ \\
\cline { 3 - 8 } & & Type-II & $(0 \star 19,5)$ & {$[2,14]$} & 0.9921 & 0.4615 & $93.33 \%$ \\
\hline \multirow{3}{*}{0.05} & \multirow{3}{*}{42504} & Best PC & $(0,0,1,1,0 \star 15,3)$ & {$[3,11]$} & 0.9501 & 0.3358 & $100.00 \%$ \\
& & Worst PC & $(0,5,0 \star 18)$ & {$[2,10]$} & 0.9754 & 0.3887 & $86.39 \%$ \\
\cline { 3 - 8 } & & Type-II & $(0 \star 19,5)$ & {$[3,12]$} & 0.9572 & 0.3462 & $96.99 \%$ \\
\hline \multirow{3}{*}{0.10} & \multirow{3}{*}{42504} & Best PC & $(0 \star 7,1,0 \star 11,4)$ & {$[3,10]$} & 0.9015 & 0.2738 & $100.00 \%$ \\
& & Worst PC & $(0,5,0 \star 18)$ & {$[3,10]$} & 0.9307 & 0.3401 & $80.50 \%$ \\
\cline { 3 - 8 } & & Type-II & $(0 \star 19,5)$ & {$[3,11]$} & 0.9382 & 0.3077 & $88.97 \%$ \\
\hline
\end{tabular}

Table A.22: Optimal PC Schemes for Non-Parametric CIs of $\xi_{0.35}$ with $n=25$ and $m=20$ (total 42504 progressive Type-II censoring schemes examined)

\begin{tabular}{|c|c|c|c|c|c|c|r|}
\hline$\alpha$ & Count & Category & Censoring Scheme (R) & CI & Actual CL & CI Mass & Efficiency \\
\hline \hline \multirow{3}{*}{0.01} & \multirow{3}{*}{42504} & Best PC & $(0 \star 11,1,0 \star 7,4)$ & {$[3,15]$} & 0.9900 & 0.4704 & $100.00 \%$ \\
& & Worst PC & $(0 \star 8,2,1 \star 3,0 \star 8)$ & {$[3,15]$} & 0.9955 & 0.5432 & $86.60 \%$ \\
\cline { 3 - 8 } & & Type-II & $(0 \star 19,5)$ & {$[3,16]$} & 0.9949 & 0.5000 & $94.08 \%$ \\
\hline \multirow{3}{*}{0.05} & \multirow{3}{*}{42504} & Best PC & $(0 \star 5,1,0 \star 6,1,0 \star 6,3)$ & {$[5,14]$} & 0.9503 & 0.3660 & $100.00 \%$ \\
& & Worst PC & $(1,2,2,0 \star 17)$ & {$[4,13]$} & 0.9684 & 0.4388 & $83.42 \%$ \\
\cline { 3 - 8 } & & Type-II & $(0 \star 19,5)$ & {$[4,14]$} & 0.9649 & 0.3846 & $95.17 \%$ \\
\hline \multirow{3}{*}{0.10} & \multirow{3}{*}{42504} & Best PC & $(0 \star 19,5)$ & {$[5,13]$} & 0.9075 & 0.3077 & $100.00 \%$ \\
& & Worst PC & $(2,1,1,0 \star 3,1,0 \star 13)$ & {$[3,11]$} & 0.9144 & 0.3820 & $80.55 \%$ \\
\cline { 3 - 8 } & & Type-II & $(0 \star 19,5)$ & {$[5,13]$} & 0.9075 & 0.3077 & $100.00 \%$ \\
\hline
\end{tabular}


Table A.23: Optimal PC Schemes for Non-Parametric CIs of $\xi_{0.50}$ with $n=25$ and $m=20$ (total 42504 progressive Type-II censoring schemes examined)

\begin{tabular}{|c|c|c|c|c|c|c|c|}
\hline$\alpha$ & Count & Category & Censoring Scheme (R) & CI & Actual CL & CI Mass & Efficiency \\
\hline \hline \multirow{3}{*}{0.01} & \multirow{3}{*}{42504} & Best PC & $(0 \star 19,5)$ & {$[7,20]$} & 0.9906 & 0.5000 & $100.00 \%$ \\
& & Worst PC & $(0,1,1,2,1,0 \star 15)$ & {$[6,18]$} & 0.9924 & 0.5956 & $83.94 \%$ \\
\cline { 3 - 8 } & & Type-II & $(0 \star 19,5)$ & {$[7,20]$} & 0.9906 & 0.5000 & $100.00 \%$ \\
\hline \multirow{3}{*}{0.05} & \multirow{3}{*}{42504} & Best PC & $(0 \star 10,2,0 \star 8,3)$ & {$[8,17]$} & 0.9501 & 0.3817 & $100.00 \%$ \\
& & Worst PC & $(0 \star 4,1,0,3,1,0 \star 12)$ & {$[7,16]$} & 0.9749 & 0.4660 & $81.90 \%$ \\
\cline { 3 - 8 } & & Type-II & $(0 \star 19,5)$ & {$[8,18]$} & 0.9567 & 0.3846 & $99.23 \%$ \\
\hline \multirow{3}{*}{0.10} & \multirow{3}{*}{42504} & West PC & $(0 \star 13,1,0 \star 5,4)$ & {$[9,17]$} & 0.9010 & 0.3182 & $100.00 \%$ \\
& & Worst PC & $(0,1,0,1 \star 4,0 \star 13)$ & {$[8,16]$} & 0.9288 & 0.4051 & $78.54 \%$ \\
\cline { 3 - 8 } & & Type-II & $(0 \star 19,5)$ & {$[8,17]$} & 0.9245 & 0.3462 & $91.92 \%$ \\
\hline
\end{tabular}

Table A.24: Optimal PC Schemes for Non-Parametric CIs of $\xi_{0.65}$ with $n=25$ and $m=20$ (total 42504 progressive Type-II censoring schemes examined)

\begin{tabular}{|c|c|c|c|c|c|c|c|}
\hline$\alpha$ & Count & Category & Censoring Scheme (R) & CI & Actual CL & CI Mass & Efficiency \\
\hline \hline \multirow{4}{*}{0.01} & \multirow{3}{*}{36505} & Best PC & $(1,0 \star 9,3,0 \star 8,1)$ & {$[10,20]$} & 0.9902 & 0.4990 & $100.00 \%$ \\
& & Worst PC & $(0 \star 7,1,0,0,1,0 \star 3,1,0,1,0,1,0)$ & {$[4,20]$} & 0.9900 & 0.7573 & $65.89 \%$ \\
\cline { 3 - 8 } & & Type-II & $(0 \star 19,5)$ & {$[1,20]$} & 0.9174 & 0.7308 & NA \\
\hline \multirow{3}{*}{0.05} & \multirow{3}{*}{42485} & Best PC & $(0 \star 15,3,0 \star 3,2)$ & {$[12,20]$} & 0.9516 & 0.3736 & $100.00 \%$ \\
& & Worst PC & $(0 \star 8,2,0 \star 7,1,2,0,0)$ & {$[11,20]$} & 0.9764 & 0.4819 & $77.53 \%$ \\
\cline { 3 - 8 } & & Type-II & $(0 \star 19,5)$ & {$[1,20]$} & 0.9174 & 0.7308 & NA \\
\hline \multirow{3}{*}{0.10} & \multirow{3}{*}{42504} & Best PC & $(0 \star 13,1,0,1,0 \star 3,3)$ & {$[13,20]$} & 0.9011 & 0.3112 & $100.00 \%$ \\
& & Worst PC & $(0 \star 7,1,0 \star 8,1,0,3,0)$ & {$[12,20]$} & 0.9458 & 0.4131 & $75.34 \%$ \\
\cline { 3 - 8 } & & Type-II & $(0 \star 19,5)$ & {$[11,20]$} & 0.9081 & 0.3462 & $89.90 \%$ \\
\hline
\end{tabular}


Table A.25: Optimal PC Schemes for Non-Parametric Cls of $\xi_{0.75}$ with $n=25$ and $m=20$ (total 42504 progressive Type-II censoring schemes examined)

\begin{tabular}{|c|c|c|c|c|c|c|c|}
\hline$\alpha$ & Count & Category & Censoring Scheme (R) & CI & Actual CL & CI Mass & Efficiency \\
\hline \multirow{3}{*}{0.01} & \multirow{3}{*}{113} & Best PC & $(0 \star 6,5,0 \star 13)$ & {$[11,20]$} & 0.9906 & 0.4698 & $100.00 \%$ \\
\hline & & Worst PC & $(0,3,1,0,1,0 \star 15)$ & {$[7,20]$} & 0.9900 & 0.6391 & $73.51 \%$ \\
\hline & & Type-II & $(0 \star 19,5)$ & {$[1,20]$} & 0.6217 & 0.7308 & NA \\
\hline \multirow{3}{*}{0.05} & \multirow{3}{*}{26247} & Best PC & $(0 \star 14,5,0 \star 5)$ & {$[15,20]$} & 0.9521 & 0.3526 & $100.00 \%$ \\
\hline & & Worst PC & $(1,1,0 \star 4,1,0 \star 9,1,0,1,0)$ & {$[7,20]$} & 0.9500 & 0.6319 & $55.79 \%$ \\
\hline & & Type-II & $(0 \star 19,5)$ & {$[1,20]$} & 0.6217 & 0.7308 & NA \\
\hline \multirow{3}{*}{0.10} & \multirow{3}{*}{39544} & Best PC & $(0 \star 8,4,0 \star 10,1)$ & {$[14,20]$} & 0.9030 & 0.3018 & $100.00 \%$ \\
\hline & & Worst PC & $(0 \star 10,2,0 \star 5,1,0,2,0)$ & {$[8,20]$} & 0.9000 & 0.5888 & $51.26 \%$ \\
\hline & & Type-II & $(0 \star 19,5)$ & {$[1,20]$} & 0.6217 & 0.7308 & NA \\
\hline
\end{tabular}

Table A.26: Optimal PC Schemes for Non-Parametric CIs of $\xi_{0.15}$ with $n=30$ and $m=8$ (total 1560780 progressive Type-II censoring schemes examined)

\begin{tabular}{|c|c|c|c|c|c|c|c|}
\hline$\alpha$ & Count & Category & Censoring Scheme (R) & CI & Actual CL & CI Mass & Efficiency \\
\hline \hline \multirow{3}{*}{0.01} & \multirow{3}{*}{145800} & Best PC & $(13,0 \star 4,1,0,8)$ & {$[1,8]$} & 0.9900 & 0.4088 & $100.00 \%$ \\
& & Worst PC & $(11,11,0 \star 6)$ & {$[1,8]$} & 0.9920 & 0.8368 & $48.86 \%$ \\
\cline { 3 - 8 } & & Type-II & $(0 \star 7,22)$ & {$[1,8]$} & 0.9226 & 0.2258 & NA \\
\hline \multirow{3}{*}{0.05} & \multirow{3}{*}{1559645} & Best PC & $(3,0 \star 6,19)$ & {$[1,8]$} & 0.9502 & 0.2509 & $100.00 \%$ \\
& & Worst PC & $(0,0,1,5,7,9,0,0)$ & {$[1,8]$} & 0.9847 & 0.7129 & $35.20 \%$ \\
\cline { 3 - 9 } & & Type-II & $(0 \star 7,22)$ & {$[1,8]$} & 0.9226 & 0.2258 & NA \\
\hline \multirow{3}{*}{0.10} & \multirow{2}{*}{1560780} & Best PC & $(0,0,2,0,0,1,0,19)$ & {$[2,8]$} & 0.9002 & 0.2091 & $100.00 \%$ \\
& & Worst PC & $(0 \star 4,3,10,9,0)$ & {$[2,8]$} & 0.9351 & 0.5678 & $36.83 \%$ \\
\cline { 3 - 8 } & & Type-II & $(0 \star 7,22)$ & {$[1,8]$} & 0.9226 & 0.2258 & $92.61 \%$ \\
\hline
\end{tabular}


Table A.27: Optimal PC Schemes for Non-Parametric CIs of $\xi_{0.25}$ with $n=30$ and $m=8$ (total 1560780 progressive Type-II censoring schemes examined)

\begin{tabular}{|c|c|c|c|c|c|c|c|}
\hline$\alpha$ & Count & Category & Censoring Scheme (R) & CI & Actual CL & CI Mass & Efficiency \\
\hline \hline \multirow{4}{*}{0.01} & \multirow{3}{*}{3078} & Best PC & $(0,18,0 \star 5,4)$ & {$[2,8]$} & 0.9903 & 0.5103 & $100.00 \%$ \\
& & Worst PC & $(18,4,0 \star 6)$ & {$[1,8]$} & 0.9979 & 0.8410 & $60.67 \%$ \\
\cline { 3 - 8 } & & Type-II & $(0 \star 7,22)$ & {$[1,8]$} & 0.5141 & 0.2258 & NA \\
\hline \multirow{3}{*}{0.05} & \multirow{3}{*}{179308} & Best PC & $(0,0,16,0 \star 4,6)$ & {$[3,8]$} & 0.9542 & 0.3763 & $100.00 \%$ \\
& & Worst PC & $(1,14,7,0 \star 5)$ & {$[2,8]$} & 0.9855 & 0.7898 & $47.65 \%$ \\
\cline { 3 - 9 } & & Type-II & $(0 \star 7,22)$ & {$[1,8]$} & 0.5141 & 0.2258 & NA \\
\hline \multirow{3}{*}{0.10} & \multirow{3}{*}{799191} & Best PC & $(0,0,13,0 \star 3,1,8)$ & {$[3,8]$} & 0.9012 & 0.3071 & $100.00 \%$ \\
& & Worst PC & $(7,8,0,2,5,0 \star 3)$ & {$[2,8]$} & 0.9634 & 0.7471 & $41.10 \%$ \\
\cline { 3 - 8 } & & Type-II & $(0 \star 7,22)$ & {$[1,8]$} & 0.5141 & 0.2258 & NA \\
\hline
\end{tabular}


Table A.28: Optimal PC Schemes for Non-Parametric CIs of $\xi_{0.35}$ with $n=30$ and $m=8$ (total 1560780 progressive Type-II censoring schemes examined)

\begin{tabular}{|c|c|c|c|c|c|c|c|}
\hline$\alpha$ & Count & Category & Censoring Scheme (R) & CI & Actual CL & CI Mass & Efficiency \\
\hline \hline \multirow{4}{*}{0.01} & \multirow{3}{*}{59} & Best PC & $(0,0,21,0 \star 4,1)$ & {$[3,8]$} & 0.9906 & 0.6452 & $100.00 \%$ \\
& & Worst PC & $(21,1,0 \star 6)$ & {$[1,8]$} & 0.9980 & 0.8449 & $76.36 \%$ \\
\cline { 3 - 8 } & & Type-II & $(0 \star 7,22)$ & {$[1,8]$} & 0.1238 & 0.2258 & NA \\
\hline \multirow{4}{*}{0.05} & \multirow{4}{*}{1592} & Best PC & $(0 \star 3,20,0 \star 3,2)$ & {$[4,8]$} & 0.9540 & 0.4977 & $100.00 \%$ \\
& & Worst PC & $(14,8,0 \star 6)$ & {$[1,8]$} & 0.9516 & 0.8381 & $59.38 \%$ \\
\cline { 3 - 8 } & & Type-II & $(0 \star 7,22)$ & {$[1,8]$} & 0.1238 & 0.2258 & NA \\
\hline \multirow{4}{*}{0.10} & \multirow{3}{*}{9863} & Best PC & $(0 \star 4,20,0,0,2)$ & {$[5,8]$} & 0.9067 & 0.4194 & $100.00 \%$ \\
& & Worst PC & $(9,9,3,1,0 \star 4)$ & {$[1,8]$} & 0.9000 & 0.8241 & $50.89 \%$ \\
\cline { 3 - 8 } & & Type-II & $(0 \star 7,22)$ & {$[1,8]$} & 0.1238 & 0.2258 & NA \\
\hline
\end{tabular}

Table A.29: Optimal PC Schemes for Non-Parametric CIs of $\xi_{0.15}$ with $n=30$ and $m=25$ (total 118755 progressive Type-II censoring schemes examined)

\begin{tabular}{|c|c|c|c|c|c|c|c|}
\hline$\alpha$ & Count & Category & Censoring Scheme (R) & CI & Actual CL & CI Mass & Efficiency \\
\hline \hline \multirow{3}{*}{0.01} & \multirow{3}{*}{118755} & Best PC & $(0 \star 3,1,0 \star 20,4)$ & {$[1,11]$} & 0.9900 & 0.3313 & $100.00 \%$ \\
& & Worst PC & $(5,0 \star 24)$ & {$[1,11]$} & 0.9918 & 0.3871 & $85.58 \%$ \\
\cline { 3 - 9 } & & Type-II & $(0 \star 24,5)$ & {$[1,12]$} & 0.9916 & 0.3548 & $93.36 \%$ \\
\hline \multirow{3}{*}{0.05} & \multirow{3}{*}{118755} & Best PC & $(3,0 \star 23,2)$ & {$[1,8]$} & 0.9502 & 0.2509 & $100.00 \%$ \\
& & Worst PC & $(0,0,1,2,2,0 \star 20)$ & {$[1,9]$} & 0.9783 & 0.2930 & $85.64 \%$ \\
\cline { 3 - 9 } & & Type-II & $(0 \star 24,5)$ & {$[1,9]$} & 0.9646 & 0.2581 & $97.22 \%$ \\
\hline \multirow{3}{*}{0.10} & \multirow{2}{*}{118755} & Best PC & $(0,0,2,0,0,1,0 \star 18,2)$ & {$[2,8]$} & 0.9002 & 0.2091 & $100.00 \%$ \\
& & Worst PC & $(3,0 \star 5,1,0 \star 17,1)$ & {$[1,8]$} & 0.9514 & 0.2527 & $82.76 \%$ \\
\cline { 3 - 9 } & & Type-II & $(0 \star 24,5)$ & {$[2,9]$} & 0.9242 & 0.2258 & $92.61 \%$ \\
\hline
\end{tabular}


Table A.30: Optimal PC Schemes for Non-Parametric CIs of $\xi_{0.25}$ with $n=30$ and $m=25$ (total 118755 progressive Type-II censoring schemes examined)

\begin{tabular}{|c|c|c|c|c|c|c|c|}
\hline$\alpha$ & Count & Category & Censoring Scheme (R) & CI & Actual CL & CI Mass & Efficiency \\
\hline \hline \multirow{3}{*}{0.01} & \multirow{3}{*}{118755} & Best PC & $(0 \star 12,1,0 \star 11,4)$ & {$[2,14]$} & 0.9902 & 0.3890 & $100.00 \%$ \\
& & Worst PC & $(2,0 \star 6,1,2,0 \star 16)$ & {$[1,13]$} & 0.9924 & 0.4405 & $88.31 \%$ \\
\cline { 3 - 8 } & & Type-II & $(0 \star 24,5)$ & {$[2,15]$} & 0.9953 & 0.4194 & $92.76 \%$ \\
\hline \multirow{3}{*}{0.05} & \multirow{3}{*}{118755} & Best PC & $(0 \star 3,1,0 \star 5,1,0 \star 14,3)$ & {$[3,12]$} & 0.9502 & 0.3038 & $100.00 \%$ \\
& & Worst PC & $(0,5,0 \star 23)$ & {$[3,12]$} & 0.9697 & 0.3508 & $86.59 \%$ \\
\cline { 3 - 9 } & & Type-II & $(0 \star 24,5)$ & {$[4,14]$} & 0.9544 & 0.3226 & $94.17 \%$ \\
\hline \multirow{3}{*}{0.10} & \multirow{2}{*}{118755} & Best PC & $(0 \star 3,2,0,0,2,0 \star 17,1)$ & {$[4,11]$} & 0.9003 & 0.2578 & $100.00 \%$ \\
& & Worst PC & $(0,5,0 \star 23)$ & {$[4,12]$} & 0.9201 & 0.3118 & $82.68 \%$ \\
\cline { 3 - 9 } & & Type-II & $(0 \star 24,5)$ & {$[4,12]$} & 0.9119 & 0.2581 & $99.90 \%$ \\
\hline
\end{tabular}

Table A.31: Optimal PC Schemes for Non-Parametric CIs of $\xi_{0.35}$ with $n=30$ and $m=25$ (total 118755 progressive Type-II censoring schemes examined)

\begin{tabular}{|c|c|c|c|c|c|c|c|}
\hline$\alpha$ & Count & Category & Censoring Scheme (R) & CI & Actual CL & CI Mass & Efficiency \\
\hline \hline \multirow{3}{*}{0.01} & \multirow{3}{*}{118755} & Best PC & $(0 \star 3,1,0 \star 11,1,0 \star 8,3)$ & {$[4,17]$} & 0.9901 & 0.4381 & $100.00 \%$ \\
& & Worst PC & $(3,2,0 \star 23)$ & {$[4,17]$} & 0.9927 & 0.5048 & $86.78 \%$ \\
\cline { 3 - 9 } & & Type-II & $(0 \star 24,5)$ & {$[5,19]$} & 0.9910 & 0.4516 & $97.00 \%$ \\
\hline \multirow{3}{*}{0.05} & \multirow{3}{*}{118755} & Best PC & $(0 \star 13,1,1,0 \star 9,3)$ & {$[6,16]$} & 0.9500 & 0.3291 & $100.00 \%$ \\
& & Worst PC & $(0,1,1,3,0 \star 21)$ & {$[4,14]$} & 0.9607 & 0.3943 & $83.46 \%$ \\
\cline { 3 - 9 } & & Type-II & $(0 \star 24,5)$ & {$[6,17]$} & 0.9644 & 0.3548 & $92.74 \%$ \\
\hline \multirow{3}{*}{0.10} & \multirow{2}{*}{118755} & Best PC & $(0 \star 6,2,0 \star 6,1,0 \star 10,2)$ & {$[7,15]$} & 0.9008 & 0.2840 & $100.00 \%$ \\
& & Worst PC & $(2,1,0 \star 10,1,0 \star 11,1)$ & {$[5,14]$} & 0.9289 & 0.3256 & $87.24 \%$ \\
\cline { 3 - 9 } & & Type-II & $(0 \star 24,5)$ & {$[7,16]$} & 0.9113 & 0.2903 & $97.84 \%$ \\
\hline
\end{tabular}


Table A.32: Optimal PC Schemes for Non-Parametric CIs of $\xi_{0.50}$ with $n=30$ and $m=25$ (total 118755 progressive Type-II censoring schemes examined)

\begin{tabular}{|c|c|c|c|c|c|c|c|}
\hline$\alpha$ & Count & Category & Censoring Scheme (R) & CI & Actual CL & CI Mass & Efficiency \\
\hline \hline \multirow{3}{*}{0.01} & \multirow{3}{*}{118755} & Best PC & $(0 \star 19,1,0 \star 4,4)$ & {$[8,22]$} & 0.9904 & 0.4581 & $100.00 \%$ \\
& & Worst PC & $(0 \star 4,1,2,2,0 \star 18)$ & {$[8,21]$} & 0.9933 & 0.5259 & $87.11 \%$ \\
\cline { 3 - 9 } & & Type-II & $(0 \star 24,5)$ & {$[8,23]$} & 0.9948 & 0.4839 & $94.67 \%$ \\
\hline \multirow{3}{*}{0.05} & \multirow{3}{*}{118755} & Best PC & $(0 \star 11,1,0,1,0 \star 10,3)$ & {$[10,20]$} & 0.9500 & 0.3505 & $100.00 \%$ \\
& & Worst PC & $(0 \star 5,1,0,0,3,1,0 \star 15)$ & {$[9,19]$} & 0.9721 & 0.4141 & $84.66 \%$ \\
\cline { 3 - 9 } & & Type-II & $(0 \star 24,5)$ & {$[10,21]$} & 0.9572 & 0.3548 & $98.79 \%$ \\
\hline \multirow{3}{*}{0.10} & \multirow{3}{*}{118755} & West PC & $(0 \star 24,5)$ & {$[11,20]$} & 0.9013 & 0.2903 & $100.00 \%$ \\
& & Worst PC & $(0,1 \star 3,0,0,1,1,0 \star 17)$ & {$[8,17]$} & 0.9066 & 0.3605 & $80.53 \%$ \\
\cline { 3 - 9 } & & Type-II & $(0 \star 24,5)$ & {$[11,20]$} & 0.9013 & 0.2903 & $100.00 \%$ \\
\hline
\end{tabular}

Table A.33: Optimal PC Schemes for Non-Parametric CIs of $\xi_{0.65}$ with $n=30$ and $m=25$ (total 118755 progressive Type-II censoring schemes examined)

\begin{tabular}{|c|c|c|c|c|c|c|c|}
\hline$\alpha$ & Count & Category & Censoring Scheme (R) & $\mathrm{CI}$ & Actual CL & CI Mass & Efficiency \\
\hline \multirow{3}{*}{0.01} & \multirow{3}{*}{118678} & Best PC & $(0 \star 12,2,0 \star 11,3)$ & {$[13,25]$} & 0.9900 & 0.4355 & $100.00 \%$ \\
\hline & & Worst PC & $(0 \star 18,1,0,1,0 \star 3,3)$ & {$[9,25]$} & 0.9901 & 0.5513 & $78.99 \%$ \\
\hline & & Type-II & $(0 \star 24,5)$ & {$[1,25]$} & 0.9767 & 0.7742 & NA \\
\hline \multirow{3}{*}{0.05} & \multirow{3}{*}{118755} & Best PC & $(0 \star 22,1,0,4)$ & {$[15,25]$} & 0.9512 & 0.3318 & $100.00 \%$ \\
\hline & & Worst PC & $(0 \star 9,1,0 \star 8,1,0,1,2,0 \star 3)$ & {$[14,24]$} & 0.9727 & 0.4142 & $80.11 \%$ \\
\hline & & Type-II & $(0 \star 24,5)$ & {$[14,25]$} & 0.9644 & 0.3548 & $93.51 \%$ \\
\hline \multirow{3}{*}{0.10} & \multirow{3}{*}{118755} & Best PC & $(0 \star 15,1,0 \star 6,1,0,3)$ & {$[16,24]$} & 0.9000 & 0.2823 & $100.00 \%$ \\
\hline & & Worst PC & $(0 \star 8,1,0,0,1,1,2,0 \star 11)$ & {$[15,23]$} & 0.9259 & 0.3562 & $79.23 \%$ \\
\hline & & Type-II & $(0 \star 24,5)$ & {$[16,25]$} & 0.9116 & 0.2903 & $97.22 \%$ \\
\hline
\end{tabular}


Table A.34: Optimal PC Schemes for Non-Parametric CIs of $\xi_{0.75}$ with $n=30$ and $m=25$ (total 118755 progressive Type-II censoring schemes examined)

\begin{tabular}{|c|c|c|c|c|c|c|c|}
\hline$\alpha$ & Count & Category & Censoring Scheme (R) & CI & Actual CL & CI Mass & Efficiency \\
\hline \hline \multirow{4}{*}{0.01} & \multirow{3}{*}{44002} & Best PC & $(0 \star 4,4,0 \star 19,1)$ & {$[14,25]$} & 0.9901 & 0.4194 & $100.00 \%$ \\
& & Worst PC & $(0 \star 7,1,0 \star 4,2,0 \star 3,1,0,1,0 \star 6)$ & {$[9,25]$} & 0.9900 & 0.6602 & $63.52 \%$ \\
\cline { 3 - 8 } & & Type-II & $(0 \star 24,5)$ & {$[1,25]$} & 0.7974 & 0.7742 & NA \\
\hline \multirow{4}{*}{0.05} & \multirow{3}{*}{116290} & Best PC & $(0 \star 19,4,0 \star 4,1)$ & {$[18,25]$} & 0.9521 & 0.3180 & $100.00 \%$ \\
& & Worst PC & $(0 \star 8,1,0 \star 11,1,1,0,2,0)$ & {$[10,25]$} & 0.9500 & 0.5872 & $54.15 \%$ \\
\cline { 3 - 8 } & & Type-II & $(0 \star 24,5)$ & {$[1,25]$} & 0.7974 & 0.7742 & NA \\
\hline \multirow{3}{*}{0.10} & \multirow{3}{*}{118642} & Best PC & $(2,0 \star 16,1,0 \star 6,2)$ & {$[18,25]$} & 0.9002 & 0.2661 & $100.00 \%$ \\
& & Worst PC & $(0 \star 15,1,0 \star 6,1,1,2)$ & {$[13,25]$} & 0.9000 & 0.4294 & $61.97 \%$ \\
\cline { 3 - 8 } & & Type-II & $(0 \star 24,5)$ & {$[1,25]$} & 0.7974 & 0.7742 & NA \\
\hline
\end{tabular}

Table A.35: Optimal PC Schemes for Non-Parametric CIs of $\xi_{0.15}$ with $n=35$ and $m=7$ (total 1344904 progressive Type-II censoring schemes examined)

\begin{tabular}{|c|c|c|c|c|c|c|c|}
\hline$\alpha$ & Count & Category & Censoring Scheme (R) & CI & Actual CL & CI Mass & Efficiency \\
\hline \hline \multirow{3}{*}{0.01} & \multirow{3}{*}{12819} & Best PC & $(20,0 \star 5,8)$ & {$[1,7]$} & 0.9905 & 0.3889 & $100.00 \%$ \\
& & Worst PC & $(22,6,0 \star 5)$ & {$[1,7]$} & 0.9957 & 0.8226 & $47.27 \%$ \\
\cline { 3 - 8 } & & Type-II & $(0 \star 6,28)$ & {$[1,7]$} & 0.7315 & 0.1667 & NA \\
\hline \multirow{3}{*}{0.05} & \multirow{3}{*}{736131} & Best PC & $(13,0 \star 4,1,14)$ & {$[1,7]$} & 0.9505 & 0.2679 & $100.00 \%$ \\
& & Worst PC & $(7,10,10,1,0 \star 3)$ & {$[1,7]$} & 0.9858 & 0.7884 & $33.98 \%$ \\
\cline { 3 - 8 } & & Type-II & $(0 \star 6,28)$ & {$[1,7]$} & 0.7315 & 0.1667 & NA \\
\hline \multirow{3}{*}{0.10} & \multirow{2}{*}{1254051} & Best PC & $(0,12,0 \star 4,16)$ & {$[2,7]$} & 0.9032 & 0.2146 & $100.00 \%$ \\
& & Worst PC & $(0,4,9,13,2,0,0)$ & {$[2,7]$} & 0.9522 & 0.7035 & $30.51 \%$ \\
\cline { 3 - 8 } & & Type-II & $(0 \star 6,28)$ & {$[1,7]$} & 0.7315 & 0.1667 & NA \\
\hline
\end{tabular}


Table A.36: Optimal PC Schemes for Non-Parametric CIs of $\xi_{0.25}$ with $n=35$ and $m=7$ (total 1344904 progressive Type-II censoring schemes examined)

\begin{tabular}{|c|c|c|c|c|c|c|c|}
\hline$\alpha$ & Count & Category & Censoring Scheme (R) & CI & Actual CL & CI Mass & Efficiency \\
\hline \hline \multirow{4}{*}{0.01} & \multirow{3}{*}{193} & Best PC & $(25,0 \star 5,3)$ & {$[1,7]$} & 0.9932 & 0.5833 & $100.00 \%$ \\
& & Worst PC & $(26,2,0 \star 5)$ & {$[1,7]$} & 0.9979 & 0.8282 & $70.43 \%$ \\
\cline { 3 - 8 } & & Type-II & $(0 \star 6,28)$ & {$[1,7]$} & 0.1919 & 0.1667 & NA \\
\hline \multirow{4}{*}{0.05} & \multirow{4}{*}{4063} & Best PC & $(0,0,24,0,0,1,3)$ & {$[3,7]$} & 0.9513 & 0.4278 & $100.00 \%$ \\
& & Worst PC & $(23,5,0 \star 5)$ & {$[1,7]$} & 0.9882 & 0.8237 & $51.93 \%$ \\
\cline { 3 - 8 } & & Type-II & $(0 \star 6,28)$ & {$[1,7]$} & 0.1919 & 0.1667 & NA \\
\hline \multirow{3}{*}{0.10} & \multirow{3}{*}{22868} & Best PC & $(21,0 \star 5,7)$ & {$[2,7]$} & 0.9051 & 0.3472 & $100.00 \%$ \\
& & Worst PC & $(11,9,8,0 \star 4)$ & {$[1,7]$} & 0.9021 & 0.7992 & $43.45 \%$ \\
\cline { 3 - 8 } & & Type-II & $(0 \star 6,28)$ & {$[1,7]$} & 0.1919 & 0.1667 & NA \\
\hline
\end{tabular}

Table A.37: Optimal PC Schemes for Non-Parametric CIs of $\xi_{0.15}$ with $n=35$ and $m=30$ (total 278256 progressive Type-II censoring schemes examined)

\begin{tabular}{|c|c|c|c|c|c|c|c|}
\hline$\alpha$ & Count & Category & Censoring Scheme (R) & CI & Actual CL & CI Mass & Efficiency \\
\hline \hline \multirow{3}{*}{0.01} & \multirow{3}{*}{278256} & Best PC & $(1,0,1,0 \star 3,1,0 \star 22,2)$ & {$[1,11]$} & 0.9900 & 0.2979 & $100.00 \%$ \\
& & Worst PC & $(0 \star 5,1,2,2,0 \star 22)$ & {$[1,12]$} & 0.9947 & 0.3331 & $89.42 \%$ \\
\cline { 3 - 8 } & & Type-II & $(0 \star 29,5)$ & {$[1,12]$} & 0.9929 & 0.3056 & $97.48 \%$ \\
\hline \multirow{3}{*}{0.05} & \multirow{3}{*}{278256} & Best PC & $(0 \star 3,1,0 \star 25,4)$ & {$[2,10]$} & 0.9500 & 0.2276 & $100.00 \%$ \\
& & Worst PC & $(5,0 \star 29)$ & {$[2,10]$} & 0.9534 & 0.2593 & $87.79 \%$ \\
\cline { 3 - 8 } & & Type-II & $(0 \star 29,5)$ & {$[2,11]$} & 0.9647 & 0.2500 & $91.04 \%$ \\
\hline \multirow{3}{*}{0.10} & \multirow{2}{*}{278256} & Best PC & $(0 \star 29,5)$ & {$[2,9]$} & 0.9068 & 0.1944 & $100.00 \%$ \\
& & Worst PC & $(5,0 \star 29)$ & {$[2,9]$} & 0.9338 & 0.2269 & $85.71 \%$ \\
\cline { 3 - 8 } & & Type-II & $(0 \star 29,5)$ & {$[2,9]$} & 0.9068 & 0.1944 & $100.00 \%$ \\
\hline
\end{tabular}


Table A.38: Optimal PC Schemes for Non-Parametric CIs of $\xi_{0.25}$ with $n=35$ and $m=30$ (total 278256 progressive Type-II censoring schemes examined)

\begin{tabular}{|c|c|c|c|c|c|c|c|}
\hline$\alpha$ & Count & Category & Censoring Scheme (R) & CI & Actual CL & CI Mass & Efficiency \\
\hline \hline \multirow{3}{*}{0.01} & \multirow{3}{*}{278256} & Best PC & $(0 \star 29,5)$ & {$[3,16]$} & 0.9905 & 0.3611 & $100.00 \%$ \\
& & Worst PC & $(4,1,0 \star 28)$ & {$[1,14]$} & 0.9906 & 0.4207 & $85.84 \%$ \\
\cline { 3 - 9 } & & Type-II & $(0 \star 29,5)$ & {$[3,16]$} & 0.9905 & 0.3611 & $100.00 \%$ \\
\hline \multirow{3}{*}{0.05} & \multirow{3}{*}{278256} & Best PC & $(0 \star 29,5)$ & {$[4,14]$} & 0.9501 & 0.2778 & $100.00 \%$ \\
& & Worst PC & $(2,1,2,0 \star 27)$ & {$[3,13]$} & 0.9681 & 0.3258 & $85.25 \%$ \\
\cline { 3 - 9 } & & Type-II & $(0 \star 29,5)$ & {$[4,14]$} & 0.9501 & 0.2778 & $100.00 \%$ \\
\hline \multirow{3}{*}{0.10} & \multirow{2}{*}{278256} & Best PC & $(0 \star 4,1,0,0,1,0 \star 21,3)$ & {$[5,13]$} & 0.9004 & 0.2351 & $100.00 \%$ \\
& & Worst PC & $(3,0 \star 7,1,0 \star 20,1)$ & {$[3,12]$} & 0.9162 & 0.2774 & $84.77 \%$ \\
\cline { 3 - 9 } & & Type-II & $(0 \star 29,5)$ & {$[5,14]$} & 0.9227 & 0.2500 & $94.06 \%$ \\
\hline
\end{tabular}

Table A.39: Optimal PC Schemes for Non-Parametric CIs of $\xi_{0.35}$ with $n=35$ and $m=30$ (total 278256 progressive Type-II censoring schemes examined)

\begin{tabular}{|c|c|c|c|c|c|c|c|}
\hline$\alpha$ & Count & Category & Censoring Scheme (R) & CI & Actual CL & CI Mass & Efficiency \\
\hline \hline \multirow{3}{*}{0.01} & \multirow{3}{*}{278256} & Best PC & $(0 \star 5,1,0 \star 23,4)$ & {$[6,20]$} & 0.9901 & 0.4023 & $100.00 \%$ \\
& & Worst PC & $(2,0,2,1,0 \star 26)$ & {$[5,19]$} & 0.9940 & 0.4572 & $87.99 \%$ \\
\cline { 3 - 9 } & & Type-II & $(0 \star 29,5)$ & {$[6,21]$} & 0.9920 & 0.4167 & $96.55 \%$ \\
\hline \multirow{3}{*}{0.05} & \multirow{3}{*}{278256} & Best PC & $(0 \star 16,1,0 \star 12,4)$ & {$[7,18]$} & 0.9505 & 0.3071 & $100.00 \%$ \\
& & Worst PC & $(1,0,0,1,3,0 \star 25)$ & {$[7,18]$} & 0.9610 & 0.3625 & $84.71 \%$ \\
\cline { 3 - 9 } & & Type-II & $(0 \star 29,5)$ & {$[8,20]$} & 0.9520 & 0.3333 & $92.13 \%$ \\
\hline \multirow{3}{*}{0.10} & \multirow{2}{*}{278256} & Best PC & $(0 \star 8,1,0 \star 20,4)$ & {$[8,17]$} & 0.9005 & 0.2585 & $100.00 \%$ \\
& & Worst PC & $(0 \star 6,5,0 \star 23)$ & {$[8,17]$} & 0.9268 & 0.3021 & $85.59 \%$ \\
\cline { 3 - 8 } & & Type-II & $(0 \star 29,5)$ & {$[8,18]$} & 0.9244 & 0.2778 & $93.08 \%$ \\
\hline
\end{tabular}


Table A.40: Optimal PC Schemes for Non-Parametric CIs of $\xi_{0.50}$ with $n=35$ and $m=30$ (total 278256 progressive Type-II censoring schemes examined)

\begin{tabular}{|c|c|c|c|c|c|c|c|}
\hline$\alpha$ & Count & Category & Censoring Scheme (R) & $\mathrm{CI}$ & Actual CL & CI Mass & Efficiency \\
\hline \multirow{3}{*}{0.01} & \multirow{3}{*}{278256} & Best PC & $(0 \star 22,1,1,0 \star 5,3)$ & {$[10,25]$} & 0.9900 & 0.4243 & $100.00 \%$ \\
\hline & & Worst PC & $(0,1 \star 3,0 \star 11,1,0,0,1,0 \star 11)$ & {$[8,23]$} & 0.9918 & 0.4826 & $87.92 \%$ \\
\hline & & Type-II & $(0 \star 29,5)$ & {$[11,27]$} & 0.9907 & 0.4444 & $95.47 \%$ \\
\hline \multirow{3}{*}{0.05} & \multirow{3}{*}{278256} & Best PC & $(0 \star 11,1,0 \star 7,1,0 \star 9,3)$ & {$[12,23]$} & 0.9502 & 0.3251 & $100.00 \%$ \\
\hline & & Worst PC & $(0 \star 9,5,0 \star 20)$ & {$[12,23]$} & 0.9602 & 0.3783 & $85.92 \%$ \\
\hline & & Type-II & $(0 \star 29,5)$ & {$[12,24]$} & 0.9590 & 0.3333 & $97.52 \%$ \\
\hline \multirow{3}{*}{0.10} & \multirow{3}{*}{278256} & Best PC & $(0 \star 12,2,0 \star 16,3)$ & {$[13,22]$} & 0.9007 & 0.2738 & $100.00 \%$ \\
\hline & & Worst PC & $(3,1,0 \star 12,1,0 \star 15)$ & {$[10,20]$} & 0.9168 & 0.3238 & $84.57 \%$ \\
\hline & & Type-II & $(0 \star 29,5)$ & {$[13,23]$} & 0.9105 & 0.2778 & $98.57 \%$ \\
\hline
\end{tabular}


Table A.41: Optimal PC Schemes for Non-Parametric CIs of $\xi_{0.65}$ with $n=35$ and $m=30$

(total 278256 progressive Type-II censoring schemes examined)

\begin{tabular}{|c|c|c|c|c|c|c|c|}
\hline$\alpha$ & Count & Category & Censoring Scheme (R) & $\mathrm{CI}$ & Actual CL & CI Mass & Efficiency \\
\hline \multirow{3}{*}{0.01} & \multirow{3}{*}{278256} & Best PC & $(0 \star 25,1,0 \star 3,4)$ & {$[16,30]$} & 0.9900 & 0.4012 & $100.00 \%$ \\
\hline & & Worst PC & $(0 \star 9,1,0 \star 13,1,2,1,0 \star 4)$ & {$[15,29]$} & 0.9952 & 0.4797 & $83.64 \%$ \\
\hline & & Type-II & $(0 \star 29,5)$ & {$[15,30]$} & 0.9920 & 0.4167 & $96.30 \%$ \\
\hline \multirow{3}{*}{0.05} & \multirow{3}{*}{278256} & Best PC & $(0 \star 27,1,0,4)$ & {$[18,29]$} & 0.9510 & 0.3095 & $100.00 \%$ \\
\hline & & Worst PC & $(0 \star 3,1,0,1 \star 3,0,0,1,0 \star 19)$ & {$[16,27]$} & 0.9608 & 0.3710 & $83.42 \%$ \\
\hline & & Type-II & $(0 \star 29,5)$ & {$[18,30]$} & 0.9606 & 0.3333 & $92.86 \%$ \\
\hline \multirow{3}{*}{0.10} & \multirow{3}{*}{278256} & Best PC & $(0 \star 22,1,0 \star 6,4)$ & {$[19,28]$} & 0.9003 & 0.2616 & $100.00 \%$ \\
\hline & & Worst PC & $(0 \star 9,1,0,1,0,1,0,1,0,0,1,0 \star 11)$ & {$[16,25]$} & 0.9198 & 0.3209 & $81.51 \%$ \\
\hline & & Type-II & $(0 \star 29,5)$ & {$[19,29]$} & 0.9149 & 0.2778 & $94.17 \%$ \\
\hline
\end{tabular}


Table A.42: Optimal PC Schemes for Non-Parametric CIs of $\xi_{0.75}$ with $n=35$ and $m=30$ (total 278256 progressive Type-II censoring schemes examined)

\begin{tabular}{|c|c|c|c|c|c|c|c|}
\hline$\alpha$ & Count & Category & Censoring Scheme (R) & CI & Actual CL & CI Mass & Efficiency \\
\hline \hline \multirow{3}{*}{0.01} & \multirow{3}{*}{254874} & Best PC & $(0 \star 7,1,0 \star 9,3,0 \star 11,1)$ & {$[19,30]$} & 0.9901 & 0.3848 & $100.00 \%$ \\
& & Worst PC & $(0 \star 5,1,0,1,0 \star 16,1,0,0,1,1,0)$ & {$[12,30]$} & 0.9900 & 0.5936 & $64.83 \%$ \\
\cline { 3 - 8 } & Type-II & $(0 \star 29,5)$ & {$[1,30]$} & 0.9024 & 0.8056 & NA \\
\hline \multirow{3}{*}{0.05} & \multirow{3}{*}{278195} & Best PC & $(0 \star 20,2,0 \star 8,3)$ & {$[21,30]$} & 0.9505 & 0.2885 & $100.00 \%$ \\
& & Worst PC & $(0 \star 24,1,0,0,1,1,2)$ & {$[16,30]$} & 0.9500 & 0.4219 & $68.38 \%$ \\
\cline { 3 - 8 } & & Type-II & $(0 \star 29,5)$ & {$[1,30]$} & 0.9024 & 0.8056 & NA \\
\hline \multirow{3}{*}{0.10} & \multirow{2}{*}{278256} & Best PC & $(0 \star 13,1,0 \star 14,1,3)$ & {$[22,30]$} & 0.9002 & 0.2386 & $100.00 \%$ \\
& & Worst PC & $(0 \star 24,1 \star 6)$ & {$[22,30]$} & 0.9391 & 0.3137 & $76.07 \%$ \\
\cline { 3 - 8 } & & Type-II & $(0 \star 29,5)$ & {$[19,30]$} & 0.9002 & 0.3056 & $78.10 \%$ \\
\hline
\end{tabular}

Table A.43: Optimal PC Schemes for Non-Parametric CIs of $\xi_{0.15}$ with $n=40$ and $m=6$ (total 575757 progressive Type-II censoring schemes examined)

\begin{tabular}{|c|c|c|c|c|c|c|c|}
\hline$\alpha$ & Count & Category & Censoring Scheme (R) & CI & Actual CL & CI Mass & Efficiency \\
\hline \hline \multirow{3}{*}{0.01} & \multirow{3}{*}{626} & Best PC & $(29,0 \star 4,5)$ & {$[1,6]$} & 0.9927 & 0.4435 & $100.00 \%$ \\
& & Worst PC & $(31,3,0 \star 4)$ & {$[1,6]$} & 0.9973 & 0.8022 & $55.28 \%$ \\
\cline { 3 - 8 } & & Type-II & $(0 \star 5,34)$ & {$[1,6]$} & 0.4310 & 0.1220 & NA \\
\hline \multirow{3}{*}{0.05} & \multirow{3}{*}{15801} & Best PC & $(23,0,0,1,0,10)$ & {$[1,6]$} & 0.9506 & 0.2958 & $100.00 \%$ \\
& & Worst PC & $(25,9,0 \star 4)$ & {$[1,6]$} & 0.9867 & 0.7935 & $37.27 \%$ \\
\cline { 3 - 8 } & & Type-II & $(0 \star 5,34)$ & {$[1,6]$} & 0.4310 & 0.1220 & NA \\
\hline \multirow{3}{*}{0.10} & \multirow{3}{*}{93276} & Best PC & $(0,23,0 \star 3,11)$ & {$[2,6]$} & 0.9085 & 0.2378 & $100.00 \%$ \\
& & Worst PC & $(19,14,1,0 \star 3)$ & {$[1,6]$} & 0.9712 & 0.7820 & $30.41 \%$ \\
\cline { 3 - 8 } & & Type-II & $(0 \star 5,34)$ & {$[1,6]$} & 0.4310 & 0.1220 & NA \\
\hline
\end{tabular}


Table A.44: Optimal PC Schemes for Non-Parametric CIs of $\xi_{0.25}$ with $n=40$ and $m=6$ (total 575757 progressive Type-II censoring schemes examined)

\begin{tabular}{|c|c|c|c|c|c|c|c|}
\hline$\alpha$ & Count & Category & Censoring Scheme $(\mathbf{R})$ & CI & Actual CL & CI Mass & Efficiency \\
\hline \hline \multirow{4}{*}{0.01} & \multirow{3}{*}{28} & Best PC & $(32,0 \star 4,2)$ & {$[1,6]$} & 0.9906 & 0.6098 & $100.00 \%$ \\
& & Worst PC & $(34,0 \star 5)$ & {$[1,6]$} & 0.9993 & 0.8130 & $75.00 \%$ \\
\cline { 3 - 8 } & & Type-II & $(0 \star 5,34)$ & {$[1,6]$} & 0.0433 & 0.1220 & NA \\
\hline \multirow{4}{*}{0.05} & \multirow{3}{*}{0.10} & Best PC & $(0,31,0 \star 3,3)$ & {$[2,6]$} & 0.9575 & 0.4756 & $100.00 \%$ \\
& \multirow{3}{*}{0.10} & Worst PC & $(31,3,0 \star 4)$ & {$[1,6]$} & 0.9849 & 0.8022 & $59.29 \%$ \\
\cline { 3 - 8 } & \multirow{3}{*}{1072} & Type-II & $(0 \star 5,34)$ & {$[1,6]$} & 0.0433 & 0.1220 & NA \\
\cline { 3 - 8 } & & Best PC & $(0,0,31,0,0,3)$ & {$[3,6]$} & 0.9076 & 0.3972 & $100.00 \%$ \\
\cline { 3 - 8 } & & Worst PC & $(30,4,0 \star 4)$ & {$[1,6]$} & 0.9747 & 0.8000 & $49.65 \%$ \\
\cline { 3 - 8 } & & Type-II & $(0 \star 5,34)$ & {$[1,6]$} & 0.0433 & 0.1220 & NA \\
\hline
\end{tabular}

Table A.45: Optimal PC Schemes for Non-Parametric CIs of $\xi_{0.15}$ with $n=40$ and $m=35$ (total 575757 progressive Type-II censoring schemes examined)

\begin{tabular}{|c|c|c|c|c|c|c|r|}
\hline$\alpha$ & Count & Category & Censoring Scheme (R) & CI & Actual CL & CI Mass & Efficiency \\
\hline \hline \multirow{4}{*}{0.01} & \multirow{3}{*}{575757} & Best PC & $(0,0,2,0 \star 31,3)$ & {$[1,12]$} & 0.9900 & 0.2805 & $100.00 \%$ \\
& & Worst PC & $(0 \star 5,1,0,1 \star 4,0 \star 24)$ & {$[1,13]$} & 0.9958 & 0.3105 & $90.32 \%$ \\
\cline { 3 - 8 } & & Type-II & $(0 \star 34,5)$ & {$[1,13]$} & 0.9942 & 0.2927 & $95.83 \%$ \\
\hline \multirow{4}{*}{0.05} & \multirow{3}{*}{575757} & Best PC & $(0,4,0 \star 5,1,0 \star 27)$ & {$[2,10]$} & 0.9502 & 0.2194 & $100.00 \%$ \\
& & Worst PC & $(4,0 \star 3,1,0 \star 30)$ & {$[1,10]$} & 0.9646 & 0.2483 & $88.36 \%$ \\
\cline { 3 - 8 } & & Type-II & $(0 \star 34,5)$ & {$[2,11]$} & 0.9580 & 0.2195 & $99.93 \%$ \\
\hline \multirow{3}{*}{0.10} & \multirow{3}{*}{575757} & Best PC & $(0 \star 3,2,0,1,0 \star 28,2)$ & {$[3,10]$} & 0.9001 & 0.1823 & $100.00 \%$ \\
& & Worst PC & $(4,0 \star 33,1)$ & {$[3,11]$} & 0.9174 & 0.2168 & $84.09 \%$ \\
\cline { 3 - 8 } & & Type-II & $(0 \star 34,5)$ & {$[3,11]$} & 0.9215 & 0.1951 & $93.44 \%$ \\
\hline
\end{tabular}


Table A.46: Optimal PC Schemes for Non-Parametric CIs of $\xi_{0.25}$ with $n=40$ and $m=35$ (total 575757 progressive Type-II censoring schemes examined)

\begin{tabular}{|c|c|c|c|c|c|c|c|}
\hline$\alpha$ & Count & Category & Censoring Scheme (R) & CI & Actual CL & CI Mass & Efficiency \\
\hline \hline \multirow{3}{*}{0.01} & \multirow{3}{*}{575757} & West PC & $(0 \star 34,5)$ & {$[4,18]$} & 0.9907 & 0.3415 & $100.00 \%$ \\
& & Worst PC & $(1,2,0 \star 3,1,1,0 \star 28)$ & {$[2,16]$} & 0.9913 & 0.3867 & $88.31 \%$ \\
\cline { 3 - 8 } & & Type-II & $(0 \star 34,5)$ & {$[4,18]$} & 0.9907 & 0.3415 & $100.00 \%$ \\
\hline \multirow{3}{*}{0.05} & \multirow{3}{*}{575757} & West PC & $(0 \star 5,3,0 \star 28,2)$ & {$[5,15]$} & 0.9500 & 0.2645 & $100.00 \%$ \\
& & Worst PC & $(3,1,1,0 \star 32)$ & {$[4,15]$} & 0.9723 & 0.3074 & $86.05 \%$ \\
\cline { 3 - 9 } & & Type-II & $(0 \star 34,5)$ & {$[5,16]$} & 0.9577 & 0.2683 & $98.58 \%$ \\
\hline \multirow{3}{*}{0.10} & \multirow{2}{*}{575757} & Best PC & $(0 \star 34,5)$ & {$[6,15]$} & 0.9023 & 0.2195 & $100.00 \%$ \\
& & Worst PC & $(0 \star 4,5,0 \star 30)$ & {$[6,15]$} & 0.9257 & 0.2549 & $86.11 \%$ \\
\cline { 3 - 8 } & & Type-II & $(0 \star 34,5)$ & {$[6,15]$} & 0.9023 & 0.2195 & $100.00 \%$ \\
\hline
\end{tabular}

Table A.47: Optimal PC Schemes for Non-Parametric CIs of $\xi_{0.35}$ with $n=40$ and $m=35$ (total 575757 progressive Type-II censoring schemes examined)

\begin{tabular}{|c|c|c|c|c|c|c|c|}
\hline$\alpha$ & Count & Category & Censoring Scheme (R) & CI & Actual CL & CI Mass & Efficiency \\
\hline \hline \multirow{3}{*}{0.01} & \multirow{3}{*}{575757} & Best PC & $(0 \star 8,1,0 \star 25,4)$ & {$[7,22]$} & 0.9900 & 0.3761 & $100.00 \%$ \\
& & Worst PC & $(0 \star 4,5,0 \star 30)$ & {$[7,22]$} & 0.9914 & 0.4249 & $88.52 \%$ \\
\cline { 3 - 8 } & & Type-II & $(0 \star 34,5)$ & {$[7,23]$} & 0.9926 & 0.3902 & $96.37 \%$ \\
\hline \multirow{3}{*}{0.05} & \multirow{3}{*}{575757} & Best PC & $(0 \star 34,5)$ & {$[9,21]$} & 0.9525 & 0.2927 & $100.00 \%$ \\
& & Worst PC & $(3,0,1,0,1,0 \star 30)$ & {$[7,19]$} & 0.9699 & 0.3362 & $87.05 \%$ \\
\cline { 3 - 8 } & & Type-II & $(0 \star 34,5)$ & {$[9,21]$} & 0.9525 & 0.2927 & $100.00 \%$ \\
\hline \multirow{3}{*}{0.10} & \multirow{2}{*}{575757} & Best PC & $(0 \star 17,1,0 \star 16,4)$ & {$[9,19]$} & 0.9014 & 0.2450 & $100.00 \%$ \\
& & Worst PC & $(0,0,1 \star 4,0,1,0 \star 27)$ & {$[9,19]$} & 0.9254 & 0.2838 & $86.33 \%$ \\
\cline { 3 - 8 } & & Type-II & $(0 \star 34,5)$ & {$[10,21]$} & 0.9183 & 0.2683 & $91.32 \%$ \\
\hline
\end{tabular}


Table A.48: Optimal PC Schemes for Non-Parametric CIs of $\xi_{0.50}$ with $n=40$ and $m=35$ (total 575757 progressive Type-II censoring schemes examined)

\begin{tabular}{|c|c|c|c|c|c|c|r|}
\hline$\alpha$ & Count & Category & Censoring Scheme (R) & CI & Actual CL & CI Mass & Efficiency \\
\hline \hline \multirow{3}{*}{0.01} & \multirow{3}{*}{575757} & Best PC & $(0 \star 21,1,0 \star 12,4)$ & {$[12,28]$} & 0.9902 & 0.3984 & $100.00 \%$ \\
& & Worst PC & $(2,1,1,0,1,0 \star 30)$ & {$[11,27]$} & 0.9527 & 0.4486 & $88.80 \%$ \\
\cline { 3 - 8 } & & Type-II & $(0 \star 34,5)$ & {$[12,29]$} & 0.9936 & 0.4146 & $96.08 \%$ \\
\hline \multirow{4}{*}{0.05} & \multirow{3}{*}{575757} & Best PC & $(0 \star 13,1,0 \star 20,4)$ & {$[14,26]$} & 0.9502 & 0.3039 & $100.00 \%$ \\
& & Worst PC & $(0 \star 8,1,0,2,2,0 \star 23)$ & {$[13,25]$} & 0.9700 & 0.3515 & $86.46 \%$ \\
\cline { 3 - 8 } & & Type-II & $(0 \star 34,5)$ & {$[15,28]$} & 0.9514 & 0.3171 & $95.86 \%$ \\
\hline \multirow{3}{*}{0.10} & \multirow{3}{*}{575757} & Best PC & $(0 \star 15,1,0 \star 6,1,0 \star 11,3)$ & {$[15,25]$} & 0.9002 & 0.2562 & $100.00 \%$ \\
& & Worst PC & $(0 \star 11,1,4,0 \star 22)$ & {$[14,24]$} & 0.9332 & 0.2965 & $86.40 \%$ \\
\cline { 3 - 8 } & & Type-II & $(0 \star 34,5)$ & {$[16,27]$} & 0.9038 & 0.2683 & $95.50 \%$ \\
\hline
\end{tabular}

Table A.49: Optimal PC Schemes for Non-Parametric CIs of $\xi_{0.65}$ with $n=40$ and $m=35$ (total 575757 progressive Type-II censoring schemes examined)

\begin{tabular}{|c|c|c|c|c|c|c|r|}
\hline$\alpha$ & Count & Category & Censoring Scheme (R) & CI & Actual CL & CI Mass & Efficiency \\
\hline \hline \multirow{3}{*}{0.01} & \multirow{3}{*}{575757} & Best PC & $(0 \star 23,1,0 \star 10,4)$ & {$[19,34]$} & 0.9900 & 0.3811 & $100.00 \%$ \\
& & Worst PC & $(0 \star 17,1,0 \star 10,2,2,0 \star 5)$ & {$[18,33]$} & 0.9949 & 0.4363 & $87.34 \%$ \\
\cline { 3 - 8 } & & Type-II & $(0 \star 34,5)$ & {$[19,35]$} & 0.9912 & 0.3902 & $97.66 \%$ \\
\hline \multirow{4}{*}{0.05} & \multirow{3}{*}{575757} & Best PC & $(0 \star 34,5)$ & {$[21,33]$} & 0.9513 & 0.2927 & $100.00 \%$ \\
& & Worst PC & $(0 \star 14,1,0,1,2,1,0 \star 16)$ & {$[20,31]$} & 0.9624 & 0.3414 & $85.74 \%$ \\
\cline { 3 - 8 } & & Type-II & $(0 \star 34,5)$ & {$[21,33]$} & 0.9513 & 0.2927 & $100.00 \%$ \\
\hline \multirow{3}{*}{0.10} & \multirow{2}{*}{575757} & Best PC & $(0 \star 29,1,0 \star 4,4)$ & {$[21,31]$} & 0.9027 & 0.2463 & $100.00 \%$ \\
& & Worst PC & $(0 \star 5,1,0 \star 3,1,1,0,1,1,0 \star 21)$ & {$[20,30]$} & 0.9205 & 0.2933 & $83.98 \%$ \\
\cline { 3 - 8 } & & Type-II & $(0 \star 34,5)$ & {$[22,33]$} & 0.9177 & 0.2683 & $91.82 \%$ \\
\hline
\end{tabular}


Table A.50: Optimal PC Schemes for Non-Parametric CIs of $\xi_{0.75}$ with $n=40$ and $m=35$ (total 575757 progressive Type-II censoring schemes examined)

\begin{tabular}{|c|c|c|c|c|c|c|c|}
\hline$\alpha$ & Count & Category & Censoring Scheme (R) & CI & Actual CL & CI Mass & Efficiency \\
\hline \hline \multirow{4}{*}{0.01} & \multirow{3}{*}{574293} & Best PC & $(0 \star 24,3,0 \star 9,2)$ & {$[23,35]$} & 0.9901 & 0.3490 & $100.00 \%$ \\
& & Worst PC & $(0 \star 20,1,0 \star 10,1,0,3,0)$ & {$[16,35]$} & 0.9900 & 0.5364 & $65.06 \%$ \\
\cline { 3 - 9 } & & Type-II & $(0 \star 34,5)$ & {$[1,35]$} & 0.9567 & 0.8293 & NA \\
\hline \multirow{3}{*}{0.05} & \multirow{3}{*}{575757} & Best PC & $(0 \star 26,1,0 \star 6,1,3)$ & {$[25,35]$} & 0.9503 & 0.2642 & $100.00 \%$ \\
& & Worst PC & $(0 \star 8,1,0 \star 23,1,3,0)$ & {$[24,35]$} & 0.9718 & 0.3294 & $80.20 \%$ \\
\cline { 3 - 9 } & & Type-II & $(0 \star 34,5)$ & {$[23,35]$} & 0.9521 & 0.2927 & $90.26 \%$ \\
\hline \multirow{3}{*}{0.10} & \multirow{3}{*}{575757} & West PC & $(0 \star 34,5)$ & {$[26,35]$} & 0.9023 & 0.2195 & $100.00 \%$ \\
& & Worst PC & $(0 \star 33,5,0)$ & {$[26,35]$} & 0.9347 & 0.2805 & $78.26 \%$ \\
\cline { 3 - 9 } & & Type-II & $(0 \star 34,5)$ & {$[26,35]$} & 0.9023 & 0.2195 & $100.00 \%$ \\
\hline
\end{tabular}

Table A.51: Optimal PC Schemes for Non-Parametric CIs of $\xi_{0.85}$ with $n=40$ and $m=35$ (total 575757 progressive Type-II censoring schemes examined)

\begin{tabular}{|c|c|c|c|c|c|c|c|}
\hline$\alpha$ & Count & Category & Censoring Scheme (R) & $\mathrm{CI}$ & Actual CL & CI Mass & Efficiency \\
\hline \multirow{3}{*}{0.01} & \multirow{3}{*}{3682} & Best PC & $(0 \star 14,5,0 \star 20)$ & {$[25,35]$} & 0.9902 & 0.3020 & $100.00 \%$ \\
\hline & & Worst PC & $(0 \star 6,3,0,0,1,0 \star 5,1,0 \star 19)$ & {$[18,35]$} & 0.9900 & 0.4952 & $60.98 \%$ \\
\hline & & Type-II & $(0 \star 34,5)$ & {$[1,35]$} & 0.5675 & 0.8293 & NA \\
\hline \multirow{3}{*}{0.05} & \multirow{3}{*}{443366} & Best PC & $(0 \star 13,4,0 \star 20,1)$ & {$[27,35]$} & 0.9500 & 0.2291 & $100.00 \%$ \\
\hline & & Worst PC & $(0 \star 18,1,1,0 \star 5,1,1,0 \star 4,1,0 \star 3)$ & {$[20,35]$} & 0.9500 & 0.4710 & $48.64 \%$ \\
\hline & & Type-II & $(0 \star 34,5)$ & {$[1,35]$} & 0.5675 & 0.8293 & NA \\
\hline \multirow{3}{*}{0.10} & \multirow{3}{*}{557320} & Best PC & $(0,4,0 \star 32,1)$ & {$[28,35]$} & 0.9007 & 0.1902 & $100.00 \%$ \\
\hline & & Worst PC & $(0,0,1,0 \star 26,1,0,0,3,0,0)$ & {$[21,35]$} & 0.9000 & 0.4203 & $45.27 \%$ \\
\hline & & Type-II & $(0 \star 34,5)$ & {$[1,35]$} & 0.5675 & 0.8293 & NA \\
\hline
\end{tabular}


Table A.52: Optimal PC Schemes for Non-Parametric CIs of $\xi_{0.15}$ with $n=45$ and $m=6$ (total 1086008 progressive Type-II censoring schemes examined)

\begin{tabular}{|c|c|c|c|c|c|c|c|}
\hline$\alpha$ & Count & Category & Censoring Scheme (R) & CI & Actual CL & CI Mass & Efficiency \\
\hline \hline \multirow{3}{*}{0.01} & \multirow{3}{*}{618} & Best PC & $(33,1,0 \star 3,5)$ & {$[1,6]$} & 0.9901 & 0.4402 & $100.00 \%$ \\
& & Worst PC & $(36,3,0 \star 4)$ & {$[1,6]$} & 0.9980 & 0.8043 & $54.73 \%$ \\
\cline { 2 - 8 } & & Type-II & $(0 \star 5,39)$ & {$[1,6]$} & 0.3135 & 0.1087 & NA \\
\hline \multirow{3}{*}{0.05} & \multirow{3}{*}{11417} & Best PC & $(0,31,0 \star 3,8)$ & {$[2,6]$} & 0.9516 & 0.2943 & $100.00 \%$ \\
& & Worst PC & $(31,8,0 \star 4)$ & {$[1,6]$} & 0.9884 & 0.7966 & $36.95 \%$ \\
\cline { 3 - 8 } & & Type-II & $(0 \star 5,39)$ & {$[1,6]$} & 0.3135 & 0.1087 & NA \\
\hline \multirow{3}{*}{0.10} & \multirow{2}{*}{61302} & Best PC & $(0,28,0 \star 3,11)$ & {$[2,6]$} & 0.9073 & 0.2391 & $100.00 \%$ \\
& & Worst PC & $(26,13,0 \star 4)$ & {$[1,6]$} & 0.9718 & 0.7929 & $30.16 \%$ \\
\cline { 3 - 8 } & & Type-II & $(0 \star 5,39)$ & {$[1,6]$} & 0.3135 & 0.1087 & NA \\
\hline
\end{tabular}

Table A.53: Optimal PC Schemes for Non-Parametric CIs of $\xi_{0.25}$ with $n=45$ and $m=6$ (total 1086008 progressive Type-II censoring schemes examined)

\begin{tabular}{|c|c|c|c|c|c|c|c|}
\hline$\alpha$ & Count & Category & Censoring Scheme (R) & CI & Actual CL & CI Mass & Efficiency \\
\hline \hline \multirow{3}{*}{0.01} & \multirow{3}{*}{28} & Best PC & $(37,0 \star 4,2)$ & {$[1,6]$} & 0.9903 & 0.6114 & $100.00 \%$ \\
& & Worst PC & $(39,0 \star 5)$ & {$[1,6]$} & 0.9993 & 0.8152 & $75.00 \%$ \\
\cline { 3 - 8 } & & Type-II & $(0 \star 5,39)$ & {$[1,6]$} & 0.0179 & 0.1087 & NA \\
\hline \multirow{3}{*}{0.05} & \multirow{3}{*}{247} & Best PC & $(0,36,0 \star 3,3)$ & {$[2,6]$} & 0.9551 & 0.4783 & $100.00 \%$ \\
& & Worst PC & $(37,2,0 \star 4)$ & {$[1,6]$} & 0.9919 & 0.8071 & $59.26 \%$ \\
\cline { 3 - 8 } & & Type-II & $(0 \star 5,39)$ & {$[1,6]$} & 0.0179 & 0.1087 & NA \\
\hline \multirow{3}{*}{0.10} & \multirow{3}{*}{939} & Best PC & $(0,0,36,0,0,3)$ & {$[3,6]$} & 0.9011 & 0.4006 & $100.00 \%$ \\
& & Worst PC & $(35,4,0 \star 4)$ & {$[1,6]$} & 0.9727 & 0.8022 & $49.94 \%$ \\
\cline { 3 - 8 } & & Type-II & $(0 \star 5,39)$ & {$[1,6]$} & 0.0179 & 0.1087 & NA \\
\hline
\end{tabular}


Table A.54: Optimal PC Schemes for Non-Parametric CIs of $\xi_{0.15}$ with $n=45$ and $m=40$ (total 1086008 progressive Type-II censoring schemes examined)

\begin{tabular}{|c|c|c|c|c|c|c|r|}
\hline$\alpha$ & Count & Category & Censoring Scheme (R) & CI & Actual CL & CI Mass & Efficiency \\
\hline \hline \multirow{3}{*}{0.01} & \multirow{3}{*}{1086008} & Best PC & $(0,0,1,0 \star 36,4)$ & {$[2,14]$} & 0.9900 & 0.2666 & $100.00 \%$ \\
& & Worst PC & $(5,0 \star 39)$ & {$[1,13]$} & 0.9947 & 0.2935 & $90.83 \%$ \\
\cline { 3 - 8 } & & Type-II & $(0 \star 39,5)$ & {$[2,15]$} & 0.9923 & 0.2826 & $94.32 \%$ \\
\hline \multirow{3}{*}{0.05} & \multirow{3}{*}{1086008} & Best PC & $(0,0,1,1,0 \star 35,3)$ & {$[3,12]$} & 0.9501 & 0.2048 & $100.00 \%$ \\
& & Worst PC & $(3,0 \star 8,1,0 \star 29,1)$ & {$[1,11]$} & 0.9568 & 0.2336 & $87.64 \%$ \\
\cline { 3 - 8 } & & Type-II & $(0 \star 39,5)$ & {$[3,13]$} & 0.9608 & 0.2174 & $94.19 \%$ \\
\hline \multirow{3}{*}{0.10} & \multirow{2}{*}{1086008} & Best PC & $(0 \star 39,5)$ & {$[3,11]$} & 0.9083 & 0.1739 & $100.00 \%$ \\
& & Worst PC & $(0,5,0 \star 38)$ & {$[3,11]$} & 0.9325 & 0.1962 & $88.64 \%$ \\
\cline { 3 - 8 } & & Type-II & $(0 \star 39,5)$ & {$[3,11]$} & 0.9083 & 0.1739 & $100.00 \%$ \\
\hline
\end{tabular}


Table A.55: Optimal PC Schemes for Non-Parametric CIs of $\xi_{0.25}$ with $n=45$ and $m=40$ (total 1086008 progressive Type-II censoring schemes examined)

\begin{tabular}{|c|c|c|c|c|c|c|c|}
\hline$\alpha$ & Count & Category & Censoring Scheme (R) & CI & Actual CL & CI Mass & Efficiency \\
\hline \hline \multirow{3}{*}{0.01} & \multirow{3}{*}{1086008} & Best PC & $(0 \star 39,5)$ & {$[5,20]$} & 0.9906 & 0.3261 & $100.00 \%$ \\
& & Worst PC & $(0,1,1,0 \star 6,1 \star 3,0 \star 28)$ & {$[3,18]$} & 0.9915 & 0.3576 & $91.18 \%$ \\
\cline { 3 - 9 } & & Type-II & $(0 \star 39,5)$ & {$[5,20]$} & 0.9906 & 0.3261 & $100.00 \%$ \\
\hline \multirow{2}{*}{0.05} & \multirow{2}{*}{1086008} & Best PC & $(0 \star 5,1,0 \star 7,1,0 \star 25,3)$ & {$[6,17]$} & 0.9501 & 0.2475 & $100.00 \%$ \\
& & Worst PC & $(2,0 \star 38,3)$ & {$[6,18]$} & 0.9639 & 0.2730 & $90.66 \%$ \\
\cline { 3 - 9 } & & Type-II & $(0 \star 39,5)$ & {$[6,18]$} & 0.9630 & 0.2609 & $94.87 \%$ \\
\hline \multirow{3}{*}{0.10} & \multirow{2}{*}{1086008} & Best PC & $(0 \star 8,3,0 \star 30,2)$ & {$[7,16]$} & 0.9001 & 0.2091 & $100.00 \%$ \\
& & Worst PC & $(0,1 \star 4,0 \star 7,1,0 \star 27)$ & {$[5,15]$} & 0.9171 & 0.2417 & $86.49 \%$ \\
\cline { 3 - 9 } & & Type-II & $(0 \star 39,5)$ & {$[7,17]$} & 0.9159 & 0.2174 & $96.18 \%$ \\
\hline
\end{tabular}

Table A.56: Optimal PC Schemes for Non-Parametric CIs of $\xi_{0.35}$ with $n=45$ and $m=40$ (total 1086008 progressive Type-II censoring schemes examined)

\begin{tabular}{|c|c|c|c|c|c|c|c|}
\hline$\alpha$ & Count & Category & Censoring Scheme $(\mathbf{R})$ & CI & Actual CL & CI Mass & Efficiency \\
\hline \hline \multirow{3}{*}{0.01} & \multirow{3}{*}{1086008} & West PC & $(0 \star 7,1,0 \star 31,4)$ & {$[8,24]$} & 0.9900 & 0.3572 & $100.00 \%$ \\
& & Worst PC & $(0,0,1,0,1,2,1,0 \star 33)$ & {$[8,24]$} & 0.9927 & 0.3969 & $90.01 \%$ \\
\cline { 3 - 9 } & & Type-II & $(0 \star 39,5)$ & {$[8,25]$} & 0.9929 & 0.3696 & $96.66 \%$ \\
\hline \multirow{3}{*}{0.05} & \multirow{2}{*}{1086008} & West PC & $(0 \star 12,2,0 \star 26,3)$ & {$[10,22]$} & 0.9501 & 0.2735 & $100.00 \%$ \\
& & Worst PC & $(0,1,0,0,1,0,1,0 \star 12,1,0 \star 19,1)$ & {$[8,21]$} & 0.9587 & 0.3059 & $89.41 \%$ \\
\cline { 3 - 9 } & & Type-II & $(0 \star 39,5)$ & {$[10,23]$} & 0.9589 & 0.2826 & $96.77 \%$ \\
\hline \multirow{2}{*}{0.10} & \multirow{2}{*}{1086008} & Best PC & $(0 \star 12,2,0 \star 5,1,0 \star 20,2)$ & {$[11,21]$} & 0.9002 & 0.2305 & $100.00 \%$ \\
& & Worst PC & $(0 \star 3,1 \star 3,0 \star 10,1,0 \star 22,1)$ & {$[9,20]$} & 0.9190 & 0.2608 & $88.38 \%$ \\
\cline { 3 - 9 } & & Type-II & $(0 \star 39,5)$ & {$[11,22]$} & 0.9149 & 0.2391 & $96.41 \%$ \\
\hline
\end{tabular}


Table A.57: Optimal PC Schemes for Non-Parametric Cls of $\xi_{0.50}$ with $n=45$ and $m=40$ (total 1086008 progressive Type-II censoring schemes examined)

\begin{tabular}{|c|c|c|c|c|c|c|c|}
\hline$\alpha$ & Count & Category & Censoring Scheme (R) & CI & Actual CL & CI Mass & Efficiency \\
\hline \hline \multirow{3}{*}{0.01} & \multirow{3}{*}{1086008} & Best PC & $(0 \star 24,1,0 \star 14,4)$ & {$[14,31]$} & 0.9901 & 0.3761 & $100.00 \%$ \\
& & Worst PC & $(1 \star 3,0,1,0,1,0 \star 33)$ & {$[13,30]$} & 0.9928 & 0.4193 & $89.69 \%$ \\
\cline { 3 - 8 } & & Type-II & $(0 \star 39,5)$ & {$[15,33]$} & 0.9907 & 0.3913 & $96.11 \%$ \\
\hline \multirow{3}{*}{0.05} & \multirow{3}{*}{1086008} & Best PC & $(0 \star 26,2,0 \star 12,3)$ & {$[16,29]$} & 0.9501 & 0.2877 & $100.00 \%$ \\
& & Worst PC & $(0 \star 7,1 \star 4,0 \star 5,1,0 \star 23)$ & {$[14,27]$} & 0.9595 & 0.3277 & $87.81 \%$ \\
\cline { 3 - 9 } & & Type-II & $(0 \star 39,5)$ & {$[17,31]$} & 0.9557 & 0.3043 & $94.54 \%$ \\
\hline \multirow{3}{*}{0.10} & \multirow{2}{*}{1086008} & Best PC & $(0 \star 24,1,0 \star 14,4)$ & {$[17,28]$} & 0.9009 & 0.2424 & $100.00 \%$ \\
& & Worst PC & $(0 \star 6,1,0 \star 3,1,0,1 \star 3,0 \star 25)$ & {$[17,28]$} & 0.9193 & 0.2810 & $86.25 \%$ \\
\cline { 3 - 9 } & & Type-II & $(0 \star 39,5)$ & {$[16,28]$} & 0.9146 & 0.2609 & $92.92 \%$ \\
\hline
\end{tabular}

Table A.58: Optimal PC Schemes for Non-Parametric CIs of $\xi_{0.65}$ with $n=45$ and $m=40$ (total 1086008 progressive Type-II censoring schemes examined)

\begin{tabular}{|c|c|c|c|c|c|c|c|}
\hline$\alpha$ & Count & Category & Censoring Scheme (R) & CI & Actual CL & CI Mass & Efficiency \\
\hline \hline \multirow{3}{*}{0.01} & \multirow{3}{*}{1086008} & Best PC & $(0 \star 10,1,0 \star 28,4)$ & {$[21,37]$} & 0.9901 & 0.3581 & $100.00 \%$ \\
& & Worst PC & $(0 \star 9,1,2,1,0,1,0 \star 26)$ & {$[19,35]$} & 0.9945 & 0.4073 & $87.90 \%$ \\
\cline { 3 - 9 } & & Type-II & $(0 \star 39,5)$ & {$[22,39]$} & 0.9901 & 0.3696 & $96.89 \%$ \\
\hline \multirow{3}{*}{0.05} & \multirow{3}{*}{1086008} & Best PC & $(0 \star 12,1,0 \star 17,1,0 \star 8,3)$ & {$[23,35]$} & 0.9500 & 0.2759 & $100.00 \%$ \\
& & Worst PC & $(0 \star 15,1,1,0,1 \star 3,0 \star 19)$ & {$[23,35]$} & 0.9554 & 0.3200 & $86.22 \%$ \\
\cline { 3 - 9 } & & Type-II & $(0 \star 39,5)$ & {$[24,37]$} & 0.9526 & 0.2826 & $97.63 \%$ \\
\hline \multirow{3}{*}{0.10} & \multirow{2}{*}{1086008} & Best PC & $(0,0,1,0 \star 22,1,0 \star 13,3)$ & {$[24,34]$} & 0.9001 & 0.2325 & $100.00 \%$ \\
& & Worst PC & $(0 \star 18,1,1,0,3,0 \star 18)$ & {$[24,34]$} & 0.9216 & 0.2723 & $85.37 \%$ \\
\cline { 3 - 9 } & & Type-II & $(0 \star 39,5)$ & {$[24,35]$} & 0.9149 & 0.2391 & $97.21 \%$ \\
\hline
\end{tabular}


Table A.59: Optimal PC Schemes for Non-Parametric CIs of $\xi_{0.75}$ with $n=45$ and $m=40$ (total 1086008 progressive Type-II censoring schemes examined)

\begin{tabular}{|c|c|c|c|c|c|c|c|}
\hline$\alpha$ & Count & Category & Censoring Scheme (R) & CI & Actual CL & CI Mass & Efficiency \\
\hline \hline \multirow{3}{*}{0.01} & \multirow{3}{*}{1085986} & West PC & $(1,0 \star 27,1,0 \star 10,3)$ & {$[26,40]$} & 0.9900 & 0.3276 & $100.00 \%$ \\
& & Worst PC & $(0 \star 29,1,0 \star 9,4)$ & {$[22,40]$} & 0.9900 & 0.4058 & $80.72 \%$ \\
\cline { 3 - 8 } & & Type-II & $(0 \star 39,5)$ & {$[1,40]$} & 0.9821 & 0.8478 & NA \\
\hline \multirow{3}{*}{0.05} & \multirow{3}{*}{1086008} & West PC & $(0 \star 19,1,0 \star 19,4)$ & {$[28,39]$} & 0.9501 & 0.2487 & $100.00 \%$ \\
& & Worst PC & $(0 \star 34,2,2,1,0 \star 3)$ & {$[26,38]$} & 0.9545 & 0.3019 & $82.37 \%$ \\
\cline { 3 - 9 } & & Type-II & $(0 \star 39,5)$ & {$[28,40]$} & 0.9630 & 0.2609 & $95.33 \%$ \\
\hline \multirow{3}{*}{0.10} & \multirow{2}{*}{1086008} & West PC & $(0 \star 3,1,0 \star 27,1,0 \star 7,3)$ & {$[29,38]$} & 0.9000 & 0.2116 & $100.00 \%$ \\
& & Worst PC & $(0 \star 22,1,1,0,1,1,0,1,0 \star 11)$ & {$[27,36]$} & 0.9203 & 0.2583 & $81.91 \%$ \\
\cline { 3 - 9 } & & Type-II & $(0 \star 39,5)$ & {$[30,40]$} & 0.9068 & 0.2174 & $97.32 \%$ \\
\hline
\end{tabular}


Table A.60: Optimal PC Schemes for Non-Parametric CIs of $\xi_{0.85}$ with $n=45$ and $m=40$

(total 1086008 progressive Type-II censoring schemes examined)

\begin{tabular}{|c|c|c|c|c|c|c|c|}
\hline$\alpha$ & Count & Category & Censoring Scheme (R) & CI & Actual CL & CI Mass & Efficiency \\
\hline \hline \multirow{3}{*}{0.01} & \multirow{3}{*}{264034} & Best PC & $(0 \star 11,5,0 \star 28)$ & {$[29,40]$} & 0.9903 & 0.2804 & $100.00 \%$ \\
& & Worst PC & $(0 \star 6,1,0,1,0 \star 9,1,0,1,0 \star 9,1,0 \star 9)$ & {$[21,40]$} & 0.9900 & 0.4984 & $56.26 \%$ \\
\cline { 3 - 8 } & & Type-II & $(0 \star 39,5)$ & {$[1,40]$} & 0.6858 & 0.8478 & NA \\
\hline \multirow{3}{*}{0.05} & \multirow{3}{*}{1040086} & Best PC & $(4,0 \star 38,1)$ & {$[31,40]$} & 0.9544 & 0.2147 & $100.00 \%$ \\
& & Worst PC & $(0 \star 24,1,0 \star 3,2,0 \star 8,1,1,0)$ & {$[24,40]$} & 0.9500 & 0.4293 & $50.02 \%$ \\
\cline { 3 - 8 } & & Type-II & $(0 \star 39,5)$ & {$[1,40]$} & 0.6858 & 0.8478 & NA \\
\hline \multirow{3}{*}{0.10} & \multirow{2}{*}{1082886} & Best PC & $(0 \star 8,1,0 \star 24,3,0 \star 5,1)$ & {$[34,40]$} & 0.9001 & 0.1843 & $100.00 \%$ \\
& & Worst PC & $(0 \star 20,1,0 \star 3,1,0 \star 12,1,0,2)$ & {$[25,40]$} & 0.9000 & 0.3671 & $50.21 \%$ \\
\cline { 3 - 9 } & & Type-II & $(0 \star 39,5)$ & {$[1,40]$} & 0.6858 & 0.8478 & NA \\
\hline
\end{tabular}


Table A.61: Optimal PC Schemes for Non-Parametric CIs of $\xi_{0.15}$ with $n=50$ and $m=6$ (total 1906884 progressive Type-II censoring schemes examined)

\begin{tabular}{|c|c|c|c|c|c|c|c|}
\hline$\alpha$ & Count & Category & Censoring Scheme (R) & CI & Actual CL & CI Mass & Efficiency \\
\hline \hline \multirow{4}{*}{0.01} & \multirow{3}{*}{605} & Best PC & $(38,1,0 \star 3,5)$ & {$[1,6]$} & 0.9900 & 0.4412 & $100.00 \%$ \\
& & Worst PC & $(41,3,0 \star 4)$ & {$[1,6]$} & 0.9983 & 0.8061 & $54.73 \%$ \\
\cline { 3 - 8 } & & Type-II & $(0 \star 5,44)$ & {$[1,6]$} & 0.2191 & 0.0980 & NA \\
\hline \multirow{4}{*}{0.05} & \multirow{3}{*}{9090} & Best PC & $(0,36,0 \star 3,8)$ & {$[2,6]$} & 0.9513 & 0.2956 & $100.00 \%$ \\
& & Worst PC & $(36,8,0 \star 4)$ & {$[1,6]$} & 0.9873 & 0.7983 & $37.03 \%$ \\
\cline { 3 - 8 } & & Type-II & $(0 \star 5,44)$ & {$[1,6]$} & 0.2191 & 0.0980 & NA \\
\hline \multirow{3}{*}{0.10} & \multirow{3}{*}{42269} & Best PC & $(0,33,0 \star 3,11)$ & {$[2,6]$} & 0.9039 & 0.2402 & $100.00 \%$ \\
& & Worst PC & $(32,12,0 \star 4)$ & {$[1,6]$} & 0.9711 & 0.7952 & $30.21 \%$ \\
\cline { 3 - 8 } & & Type-II & $(0 \star 5,44)$ & {$[1,6]$} & 0.2191 & 0.0980 & NA \\
\hline
\end{tabular}

Table A.62: Optimal PC Schemes for Non-Parametric CIs of $\xi_{0.25}$ with $n=50$ and $m=6$ (total 1906884 progressive Type-II censoring schemes examined)

\begin{tabular}{|c|c|c|c|c|c|c|c|}
\hline$\alpha$ & Count & Category & Censoring Scheme (R) & CI & Actual CL & CI Mass & Efficiency \\
\hline \hline \multirow{4}{*}{0.01} & & Best PC & $(42,0 \star 4,2)$ & {$[1,6]$} & 0.9900 & 0.6127 & $100.00 \%$ \\
& 28 & Worst PC & $(44,0 \star 5)$ & {$[1,6]$} & 0.9993 & 0.8170 & $75.00 \%$ \\
\cline { 3 - 8 } & & Type-II & $(0 \star 5,44)$ & {$[1,6]$} & 0.0070 & 0.0980 & NA \\
\hline \multirow{3}{*}{0.05} & \multirow{3}{*}{215} & Best PC & $(0,41,0 \star 3,3)$ & {$[2,6]$} & 0.9530 & 0.4804 & $100.00 \%$ \\
& & Worst PC & $(42,2,0 \star 4)$ & {$[1,6]$} & 0.9915 & 0.8088 & $59.39 \%$ \\
\cline { 3 - 8 } & & Type-II & $(0 \star 5,44)$ & {$[1,6]$} & 0.0070 & 0.0980 & NA \\
\hline \multirow{3}{*}{0.10} & \multirow{2}{*}{825} & Best PC & $(38,0 \star 4,6)$ & {$[1,6]$} & 0.9069 & 0.4085 & $100.00 \%$ \\
& & Worst PC & $(40,4,0 \star 4)$ & {$[1,6]$} & 0.9710 & 0.8039 & $50.81 \%$ \\
\cline { 3 - 8 } & & Type-II & $(0 \star 5,44)$ & {$[1,6]$} & 0.0070 & 0.0980 & NA \\
\hline
\end{tabular}


Table A.63: Optimal PC Schemes for Non-Parametric CIs of $\xi_{0.10}$ with $n=50$ and $m=45$ (total 1906884 progressive Type-II censoring schemes examined)

\begin{tabular}{|c|c|c|c|c|c|c|r|}
\hline$\alpha$ & Count & Category & Censoring Scheme (R) & CI & Actual CL & CI Mass & Efficiency \\
\hline \hline \multirow{3}{*}{0.01} & \multirow{3}{*}{1906884} & Best PC & $(4,0 \star 5,1,0 \star 38)$ & {$[1,11]$} & 0.9901 & 0.2153 & $100.00 \%$ \\
& & Worst PC & $(1,1,3,0 \star 42)$ & {$[1,12]$} & 0.9934 & 0.2372 & $90.78 \%$ \\
\cline { 3 - 8 } & & Type-II & $(0 \star 44,5)$ & {$[1,12]$} & 0.9916 & 0.2157 & $99.83 \%$ \\
\hline \multirow{4}{*}{0.05} & \multirow{3}{*}{0.1906884} & Best PC & $(2,0 \star 3,1,0 \star 39,2)$ & {$[1,9]$} & 0.9500 & 0.1653 & $100.00 \%$ \\
& & Worst PC & $(0,0,1,1,0,2,1,0 \star 38)$ & {$[1,10]$} & 0.9775 & 0.1877 & $88.06 \%$ \\
\cline { 3 - 8 } & & Type-II & $(0 \star 44,5)$ & {$[2,11]$} & 0.9569 & 0.1765 & $93.67 \%$ \\
\hline \multirow{3}{*}{0.10} & \multirow{2}{*}{1906884} & Best PC & $(0 \star 44,5)$ & {$[2,9]$} & 0.9083 & 0.1373 & $100.00 \%$ \\
& & Worst PC & $(5,0 \star 44)$ & {$[2,9]$} & 0.9231 & 0.1525 & $90.00 \%$ \\
\cline { 3 - 8 } & & Type-II & $(0 \star 44,5)$ & {$[2,9]$} & 0.9083 & 0.1373 & $100.00 \%$ \\
\hline
\end{tabular}

Table A.64: Optimal PC Schemes for Non-Parametric CIs of $\xi_{0.15}$ with $n=50$ and $m=45$ (total 1906884 progressive Type-II censoring schemes examined)

\begin{tabular}{|c|c|c|c|c|c|c|c|}
\hline$\alpha$ & Count & Category & Censoring Scheme (R) & CI & Actual CL & CI Mass & Efficiency \\
\hline \hline \multirow{3}{*}{0.01} & \multirow{3}{*}{1906884} & Best PC & $(0,3,0 \star 5,1,0 \star 4,1,0 \star 32)$ & {$[2,14]$} & 0.9900 & 0.2545 & $100.00 \%$ \\
& & Worst PC & $(4,0 \star 10,1,0 \star 33)$ & {$[1,14]$} & 0.9936 & 0.2783 & $91.44 \%$ \\
\cline { 3 - 8 } & & Type-II & $(0 \star 44,5)$ & {$[2,15]$} & 0.9918 & 0.2549 & $99.84 \%$ \\
\hline \multirow{3}{*}{0.05} & \multirow{3}{*}{1906884} & Best PC & $(0 \star 44,5)$ & {$[3,13]$} & 0.9558 & 0.1961 & $100.00 \%$ \\
& & Worst PC & $(0,5,0 \star 43)$ & {$[3,13]$} & 0.9677 & 0.2184 & $89.80 \%$ \\
\cline { 3 - 8 } & & Type-II & $(0 \star 44,5)$ & {$[3,13]$} & 0.9558 & 0.1961 & $100.00 \%$ \\
\hline \multirow{3}{*}{0.10} & \multirow{2}{*}{1906884} & Best PC & $(0 \star 4,1,0 \star 3,1,0,2,0 \star 33,1)$ & {$[4,12]$} & 0.9000 & 0.1626 & $100.00 \%$ \\
& & Worst PC & $(3,0 \star 7,1,0 \star 35,1)$ & {$[2,11]$} & 0.9116 & 0.1888 & $86.09 \%$ \\
\cline { 3 - 8 } & & Type-II & $(0 \star 44,5)$ & {$[4,13]$} & 0.9239 & 0.1765 & $92.12 \%$ \\
\hline
\end{tabular}


Table A.65: Optimal PC Schemes for Non-Parametric CIs of $\xi_{0.25}$ with $n=50$ and $m=45$ (total 1906884 progressive Type-II censoring schemes examined)

\begin{tabular}{|c|c|c|c|c|c|c|c|}
\hline$\alpha$ & Count & Category & Censoring Scheme (R) & $\mathrm{CI}$ & Actual CL & CI Mass & Efficiency \\
\hline \multirow{3}{*}{0.01} & \multirow{3}{*}{1906884} & Best PC & $(0 \star 6,3,0 \star 37,2)$ & {$[5,20]$} & 0.9901 & 0.3128 & $100.00 \%$ \\
\hline & & Worst PC & $(0 \star 10,1 \star 5,0 \star 30)$ & {$[4,20]$} & 0.9916 & 0.3341 & $93.61 \%$ \\
\hline & & Type-II & $(0 \star 44,5)$ & {$[5,21]$} & 0.9916 & 0.3137 & $99.70 \%$ \\
\hline \multirow{3}{*}{0.05} & \multirow{3}{*}{1906884} & Best PC & $(0 \star 44,5)$ & {$[7,19]$} & 0.9519 & 0.2353 & $100.00 \%$ \\
\hline & & Worst PC & $(0,0,1,0,1,3,0 \star 39)$ & {$[7,19]$} & 0.9635 & 0.2642 & $89.06 \%$ \\
\hline & & Type-II & $(0 \star 44,5)$ & {$[7,19]$} & 0.9519 & 0.2353 & $100.00 \%$ \\
\hline \multirow{3}{*}{0.10} & \multirow{3}{*}{1906884} & Best PC & $(0 \star 16,1,0 \star 27,4)$ & {$[8,18]$} & 0.9004 & 0.1967 & $100.00 \%$ \\
\hline & & Worst PC & $(0 \star 6,5,0 \star 38)$ & {$[8,18]$} & 0.9219 & 0.2212 & $88.90 \%$ \\
\hline & & Type-II & $(0 \star 44,5)$ & {$[8,19]$} & 0.9260 & 0.2157 & $91.18 \%$ \\
\hline
\end{tabular}

Table A.66: Optimal PC Schemes for Non-Parametric CIs of $\xi_{0.35}$ with $n=50$ and $m=45$ (total 1906884 progressive Type-II censoring schemes examined)

\begin{tabular}{|c|c|c|c|c|c|c|c|}
\hline$\alpha$ & Count & Category & Censoring Scheme (R) & CI & Actual CL & CI Mass & Efficiency \\
\hline \hline \multirow{3}{*}{0.01} & \multirow{3}{*}{1906884} & Best PC & $(1,0 \star 21,1,0 \star 21,3)$ & {$[9,26]$} & 0.9900 & 0.3424 & $100.00 \%$ \\
& & Worst PC & $(0,1,0,1 \star 3,0,1,0 \star 37)$ & {$[9,26]$} & 0.9931 & 0.3742 & $91.51 \%$ \\
\cline { 3 - 8 } & & Type-II & $(0 \star 44,5)$ & {$[10,28]$} & 0.9914 & 0.3529 & $97.03 \%$ \\
\hline \multirow{3}{*}{0.05} & \multirow{3}{*}{1906884} & Best PC & $(0 \star 5,1,0 \star 38,4)$ & {$[11,24]$} & 0.9500 & 0.2607 & $100.00 \%$ \\
& & Worst PC & $(0 \star 6,1,0,2,2,0 \star 35)$ & {$[11,24]$} & 0.9650 & 0.2894 & $90.07 \%$ \\
\cline { 3 - 9 } & & Type-II & $(0 \star 44,5)$ & {$[12,26]$} & 0.9557 & 0.2745 & $94.97 \%$ \\
\hline \multirow{3}{*}{0.10} & \multirow{2}{*}{1906884} & Best PC & $(0 \star 19,1,1,0 \star 23,3)$ & {$[12,23]$} & 0.9001 & 0.2191 & $100.00 \%$ \\
& & Worst PC & $(0 \star 9,2,3,0 \star 34)$ & {$[13,24]$} & 0.9014 & 0.2462 & $89.00 \%$ \\
\cline { 3 - 8 } & & Type-II & $(0 \star 44,5)$ & {$[13,25]$} & 0.9132 & 0.2353 & $93.12 \%$ \\
\hline
\end{tabular}


Table A.67: Optimal PC Schemes for Non-Parametric CIs of $\xi_{0.50}$ with $n=50$ and $m=45$ (total 1906884 progressive Type-II censoring schemes examined)

\begin{tabular}{|c|c|c|c|c|c|c|c|}
\hline$\alpha$ & Count & Category & Censoring Scheme (R) & CI & Actual CL & CI Mass & Efficiency \\
\hline \hline \multirow{4}{*}{0.01} & \multirow{3}{*}{1906884} & Best PC & $(0 \star 31,2,0 \star 12,3)$ & {$[16,34]$} & 0.9900 & 0.3576 & $100.00 \%$ \\
& & Worst PC & $(1,1,0,1,1,0,0,1,0 \star 37)$ & {$[15,33]$} & 0.9927 & 0.3953 & $90.46 \%$ \\
\cline { 3 - 9 } & & Type-II & $(0 \star 44,5)$ & {$[17,36]$} & 0.9910 & 0.3725 & $95.98 \%$ \\
\hline \multirow{3}{*}{0.05} & \multirow{3}{*}{1906884} & Best PC & $(0 \star 44,5)$ & {$[18,32]$} & 0.9511 & 0.2745 & $100.00 \%$ \\
& & Worst PC & $(0,1,0,1,2,1,0 \star 39)$ & {$[17,31]$} & 0.9620 & 0.3076 & $89.24 \%$ \\
\cline { 3 - 9 } & & Type-II & $(0 \star 44,5)$ & {$[18,32]$} & 0.9511 & 0.2745 & $100.00 \%$ \\
\hline \multirow{3}{*}{0.10} & \multirow{3}{*}{1906884} & West PC & $(0 \star 19,1,1,0 \star 8,1,0 \star 14,2)$ & {$[20,31]$} & 0.9001 & 0.2313 & $100.00 \%$ \\
& & Worst PC & $(1,2,0,1,1,0 \star 40)$ & {$[18,30]$} & 0.9210 & 0.2626 & $88.07 \%$ \\
\cline { 3 - 9 } & & Type-II & $(0 \star 44,5)$ & {$[20,32]$} & 0.9081 & 0.2353 & $98.29 \%$ \\
\hline
\end{tabular}

Table A.68: Optimal PC Schemes for Non-Parametric CIs of $\xi_{0.65}$ with $n=50$ and $m=45$ (total 1906884 progressive Type-II censoring schemes examined)

\begin{tabular}{|c|c|c|c|c|c|c|c|}
\hline$\alpha$ & Count & Category & Censoring Scheme (R) & CI & Actual CL & CI Mass & Efficiency \\
\hline \hline \multirow{3}{*}{0.01} & \multirow{3}{*}{1906884} & Best PC & $(0 \star 36,1,0 \star 7,4)$ & {$[24,41]$} & 0.9901 & 0.3394 & $100.00 \%$ \\
& & Worst PC & $(0 \star 4,1,0 \star 5,1 \star 3,0 \star 12,1,0 \star 19)$ & {$[21,38]$} & 0.9919 & 0.3831 & $88.60 \%$ \\
\cline { 3 - 9 } & & Type-II & $(0 \star 44,5)$ & {$[23,41]$} & 0.9914 & 0.3529 & $96.15 \%$ \\
\hline \multirow{3}{*}{0.05} & \multirow{3}{*}{1906884} & Best PC & $(0 \star 4,1,0 \star 39,4)$ & {$[26,39]$} & 0.9500 & 0.2606 & $100.00 \%$ \\
& & Worst PC & $(0 \star 13,1 \star 4,0,0,1,0 \star 25)$ & {$[23,36]$} & 0.9524 & 0.2984 & $87.31 \%$ \\
\cline { 3 - 9 } & & Type-II & $(0 \star 44,5)$ & {$[27,41]$} & 0.9537 & 0.2745 & $94.92 \%$ \\
\hline \multirow{3}{*}{0.10} & \multirow{2}{*}{1906884} & Best PC & $(0 \star 34,1,0 \star 9,4)$ & {$[27,38]$} & 0.9006 & 0.2196 & $100.00 \%$ \\
& & Worst PC & $(0 \star 13,1,1,0,0,1,0,1,1,0 \star 24)$ & {$[26,37]$} & 0.9240 & 0.2543 & $86.35 \%$ \\
\cline { 3 - 9 } & & Type-II & $(0 \star 44,5)$ & {$[26,38]$} & 0.9132 & 0.2353 & $93.33 \%$ \\
\hline
\end{tabular}


Table A.69: Optimal PC Schemes for Non-Parametric CIs of $\xi_{0.75}$ with $n=50$ and $m=45$ (total 1906884 progressive Type-II censoring schemes examined)

\begin{tabular}{|c|c|c|c|c|c|c|r|}
\hline$\alpha$ & Count & Category & Censoring Scheme (R) & CI & Actual CL & CI Mass & Efficiency \\
\hline \hline \multirow{3}{*}{0.01} & \multirow{3}{*}{1906884} & Best PC & $(0 \star 33,1,0 \star 10,4)$ & {$[30,45]$} & 0.9901 & 0.3076 & $100.00 \%$ \\
& & Worst PC & $(0 \star 19,1,0 \star 23,4,0)$ & {$[29,45]$} & 0.9951 & 0.3647 & $84.34 \%$ \\
\cline { 3 - 8 } & & Type-II & $(0 \star 44,5)$ & {$[29,45]$} & 0.9903 & 0.3137 & $98.05 \%$ \\
\hline \multirow{4}{*}{0.05} & \multirow{3}{*}{1906884} & Best PC & $(0 \star 44,5)$ & {$[32,44]$} & 0.9519 & 0.2353 & $100.00 \%$ \\
& & Worst PC & $(0 \star 28,1,2,0,0,1,1,0 \star 11)$ & {$[30,41]$} & 0.9606 & 0.2784 & $84.53 \%$ \\
\cline { 3 - 8 } & & Type-II & $(0 \star 44,5)$ & {$[32,44]$} & 0.9519 & 0.2353 & $100.00 \%$ \\
\hline \multirow{3}{*}{0.10} & \multirow{2}{*}{1906884} & Best PC & $(0 \star 41,1,0,0,4)$ & {$[33,43]$} & 0.9029 & 0.1985 & $100.00 \%$ \\
& & Worst PC & $(0 \star 21,1,1,0,1,1,0 \star 10,1,0 \star 8)$ & {$[30,40]$} & 0.9198 & 0.2382 & $83.34 \%$ \\
\cline { 3 - 8 } & & Type-II & $(0 \star 44,5)$ & {$[33,44]$} & 0.9255 & 0.2157 & $92.05 \%$ \\
\hline
\end{tabular}


Table A.70: Optimal PC Schemes for Non-Parametric CIs of $\xi_{0.85}$ with $n=50$ and $m=45$

(total 1906884 progressive Type-II censoring schemes examined)

\begin{tabular}{|c|c|c|c|c|c|c|c|}
\hline$\alpha$ & Count & Category & Censoring Scheme (R) & $\mathrm{CI}$ & Actual CL & CI Mass & Efficiency \\
\hline \multirow{3}{*}{0.01} & \multirow{3}{*}{1259139} & Best PC & $(0 \star 36,5,0 \star 8)$ & {$[36,45]$} & 0.9903 & 0.2636 & $100.00 \%$ \\
\hline & & Worst PC & $(0 \star 8,1,0 \star 6,1,0 \star 6,1,0 \star 15,1,1,0 \star 5)$ & {$[23,45]$} & 0.9900 & 0.5098 & $51.71 \%$ \\
\hline & & Type-II & $(0 \star 44,5)$ & {$[1,45]$} & 0.7806 & 0.8627 & NA \\
\hline \multirow{3}{*}{0.05} & \multirow{3}{*}{1898085} & Best PC & $(0 \star 18,3,0 \star 23,1,0,1)$ & {$[36,45]$} & 0.9500 & 0.2055 & $100.00 \%$ \\
\hline & & Worst PC & $(0 \star 22,1,0 \star 11,1,0 \star 8,3,0)$ & {$[29,45]$} & 0.9500 & 0.3725 & $55.17 \%$ \\
\hline & & Type-II & $(0 \star 44,5)$ & {$[1,45]$} & 0.7806 & 0.8627 & NA \\
\hline \multirow{3}{*}{0.10} & \multirow{3}{*}{1906588} & Best PC & $(2,0 \star 42,1,2)$ & {$[37,45]$} & 0.9016 & 0.1685 & $100.00 \%$ \\
\hline & & Worst PC & $(0 \star 24,1,0 \star 17,1,1,2)$ & {$[32,45]$} & 0.9002 & 0.2778 & $60.65 \%$ \\
\hline & & Type-II & $(0 \star 44,5)$ & {$[1,45]$} & 0.7806 & 0.8627 & NA \\
\hline
\end{tabular}


Table A.71: Optimal PC Schemes for Non-Parametric CIs of $\xi_{0.90}$ with $n=50$ and $m=45$ (total 1906884 progressive Type-II censoring schemes examined)

\begin{tabular}{|c|c|c|c|c|c|c|c|}
\hline$\alpha$ & Count & Category & Censoring Scheme (R) & $\mathrm{CI}$ & Actual CL & CI Mass & Efficiency \\
\hline \multirow{3}{*}{0.01} & \multirow{3}{*}{10} & Best PC & $(5,0 \star 44)$ & {$[33,45]$} & 0.9909 & 0.2614 & $100.00 \%$ \\
\hline & & Worst PC & $(0 \star 9,5,0 \star 35)$ & {$[32,45]$} & 0.9901 & 0.2903 & $90.06 \%$ \\
\hline & & Type-II & $(0 \star 44,5)$ & {$[1,45]$} & 0.3839 & 0.8627 & NA \\
\hline \multirow{3}{*}{0.05} & \multirow{3}{*}{830821} & Best PC & $(5,0 \star 44)$ & {$[37,45]$} & 0.9596 & 0.1743 & $100.00 \%$ \\
\hline & & Worst PC & $(0 \star 8,1,0 \star 17,2,0 \star 8,2,0 \star 9)$ & {$[29,45]$} & 0.9500 & 0.3916 & $44.51 \%$ \\
\hline & & Type-II & $(0 \star 44,5)$ & {$[1,45]$} & 0.3839 & 0.8627 & NA \\
\hline \multirow{3}{*}{0.10} & \multirow{3}{*}{1627876} & Best PC & $(4,0 \star 43,1)$ & {$[38,45]$} & 0.9157 & 0.1492 & $100.00 \%$ \\
\hline & & Worst PC & $(0 \star 8,1,0 \star 10,1,1,0 \star 18,1,0 \star 3,1,0)$ & {$[30,45]$} & 0.9000 & 0.3509 & $42.51 \%$ \\
\hline & & Type-II & $(0 \star 44,5)$ & {$[1,45]$} & 0.3839 & 0.8627 & $\mathrm{NA}$ \\
\hline
\end{tabular}




\section{Appendix B}

\section{Partial R Codes}

\section{for Numerical Study}

The programs written in $\mathrm{R}$ are provided here for conducting the numerical studies in Section 5.2 to search for the optimal progressive Type-II censoring schemes. For an illustration purpose, the values of $n=15$ and $m=8$ are used throughout the programs.

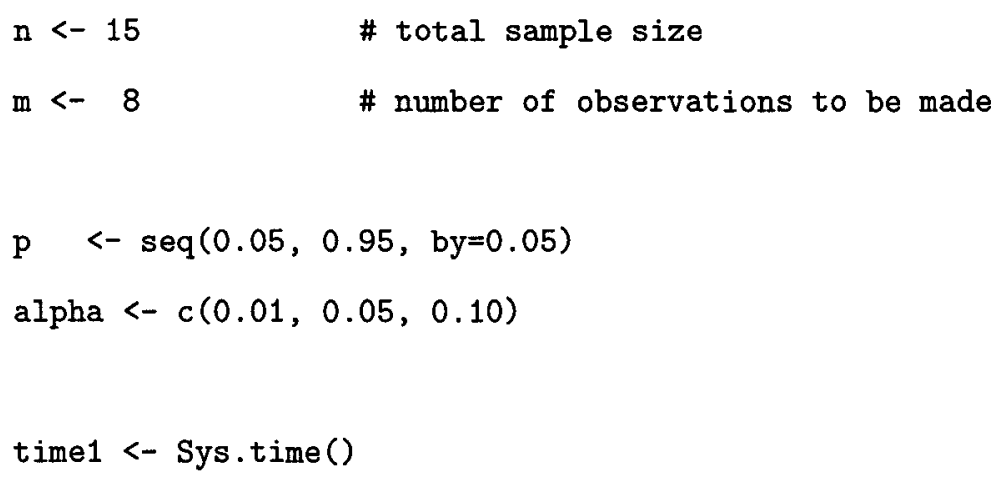




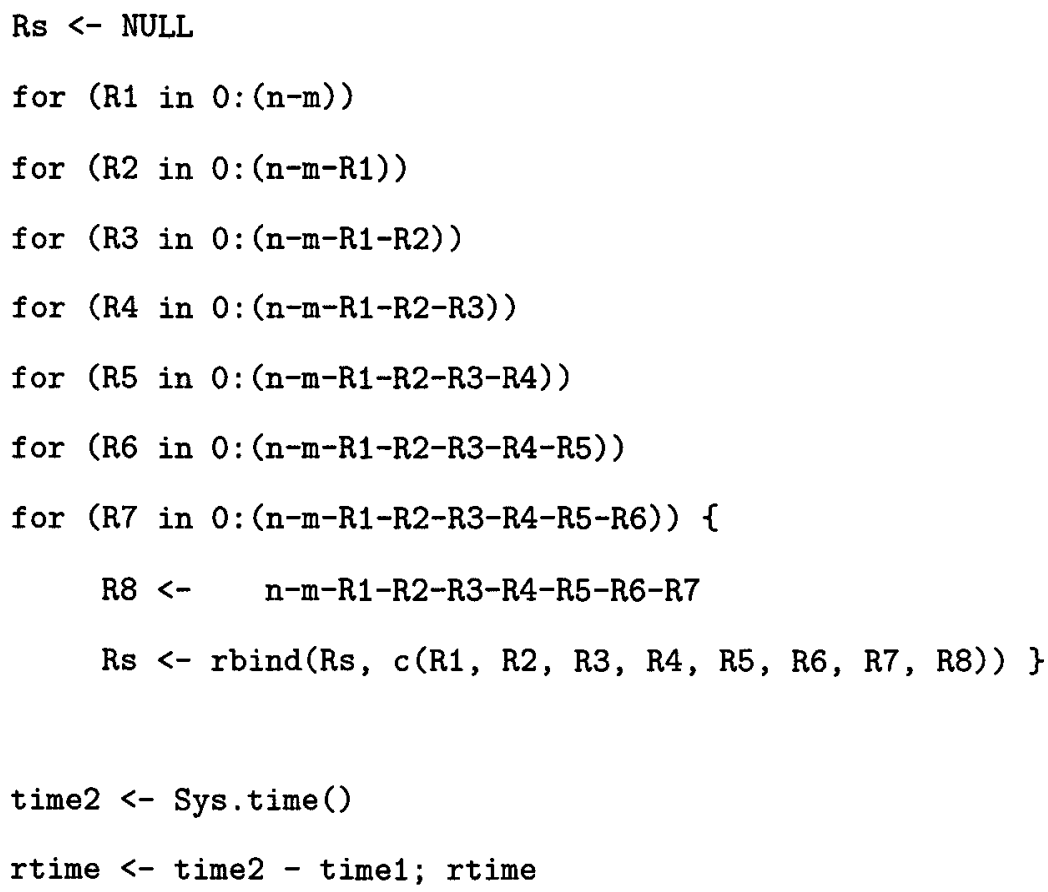


arr <- rbind(arr, rows) \}

$\operatorname{arr}<-\operatorname{arr}[,-1]$

time2 <- Sys.time()

ctime <- time2 - time1; ctime 


\section{Appendix C}

\section{Partial FORTRAN Codes for Numerical Study}

The programs written in FORTRAN are given here for conducting the numerical studies in Section 5.2 to search for the optimal progressive Type-II censoring schemes. Again, for illustration, the values of $n=15$ and $m=8$ are used throughout the programs.

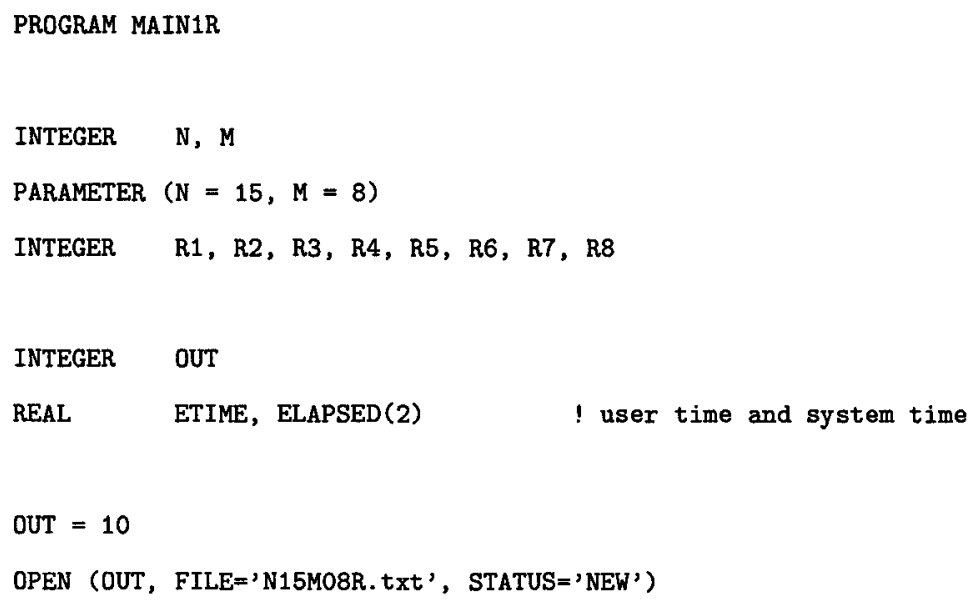




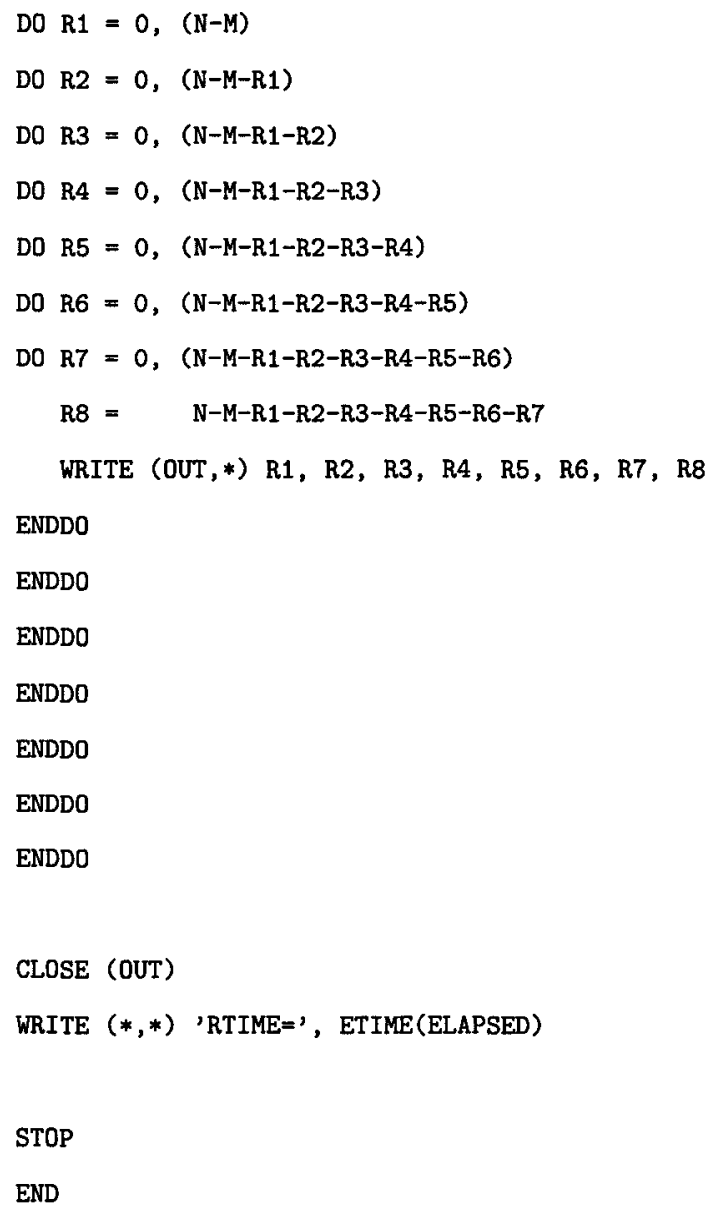




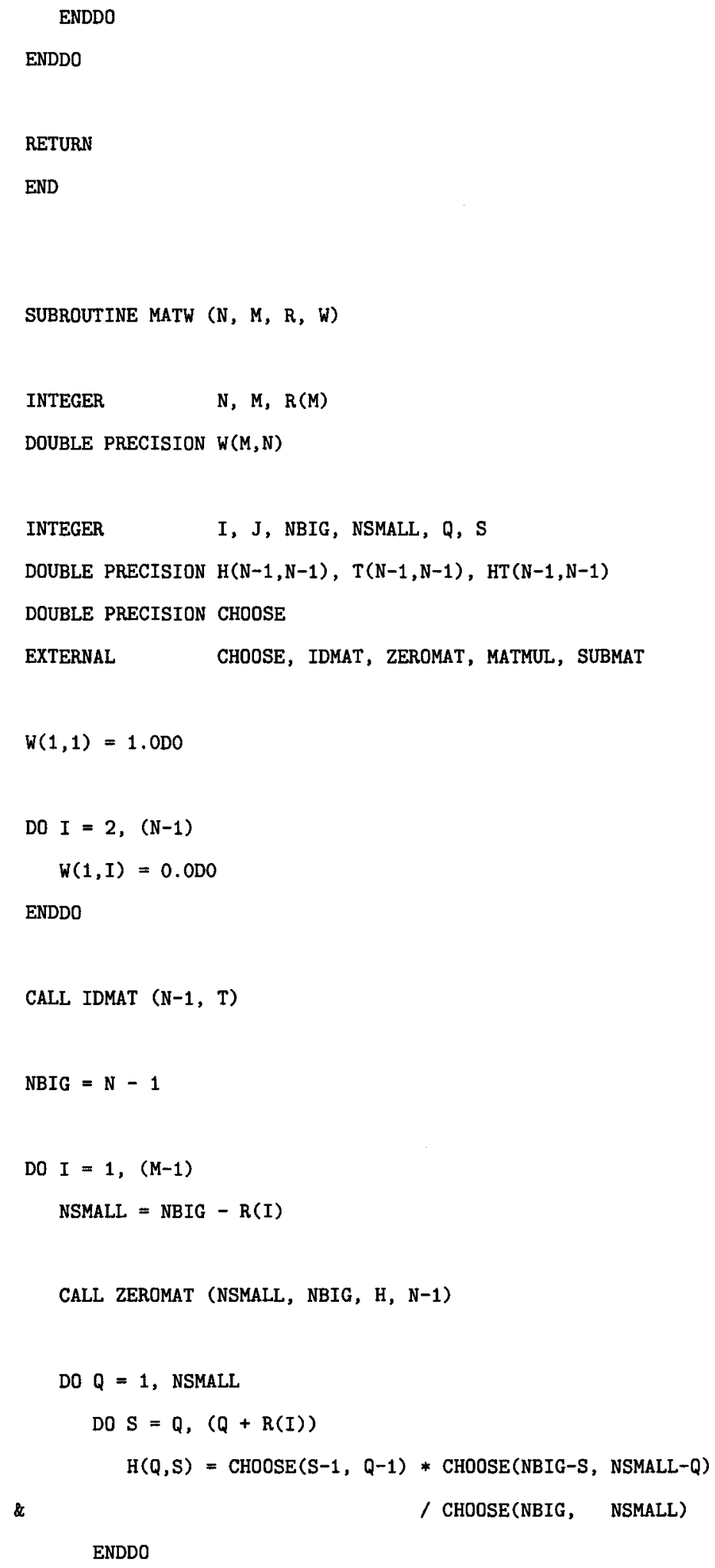




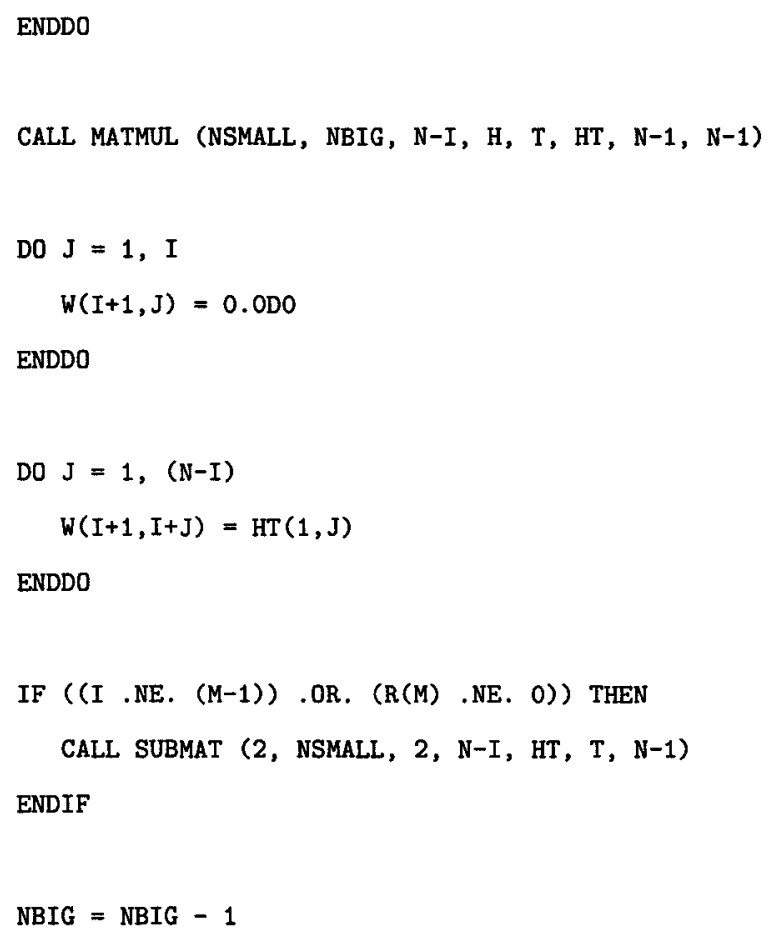




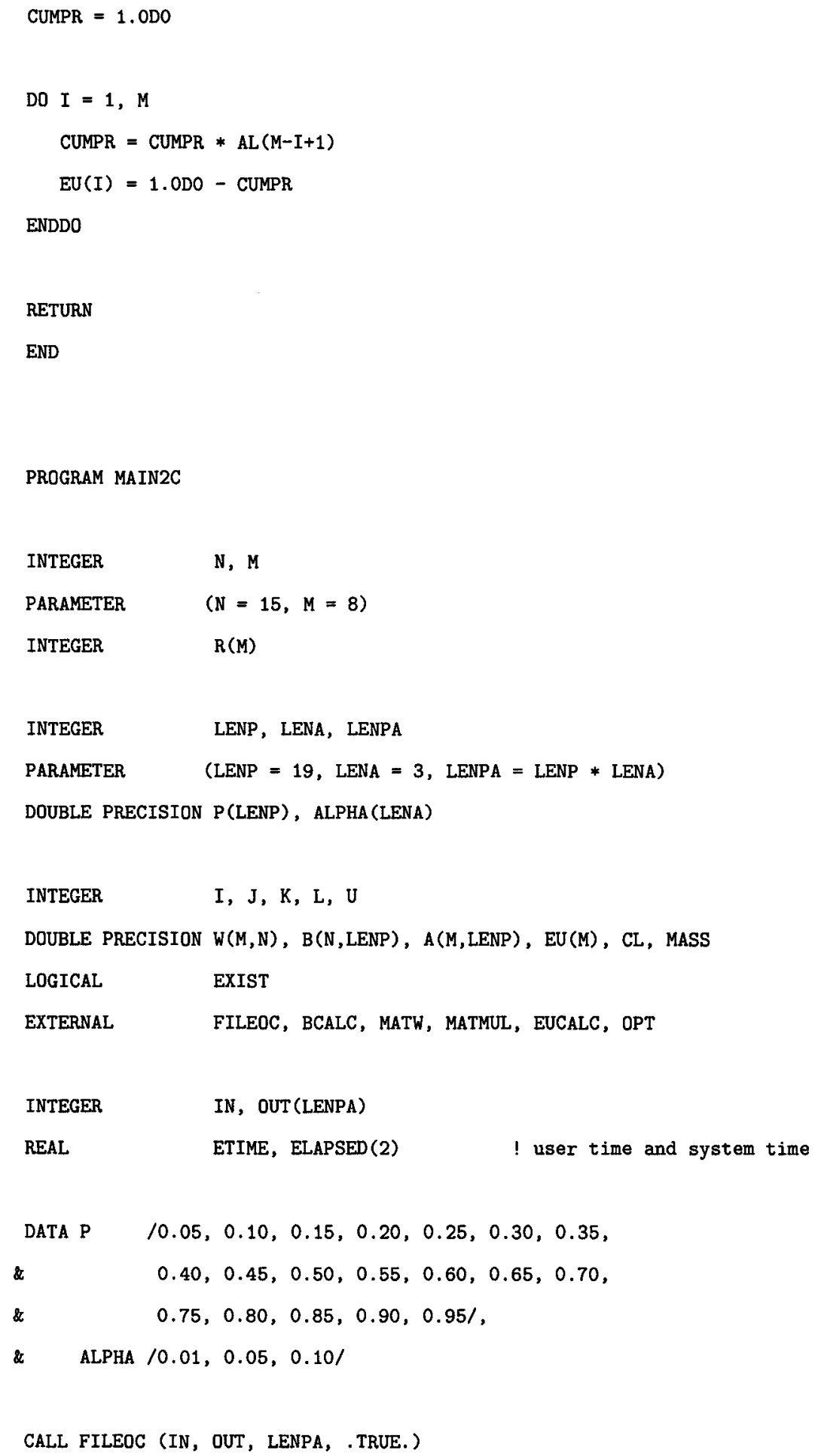




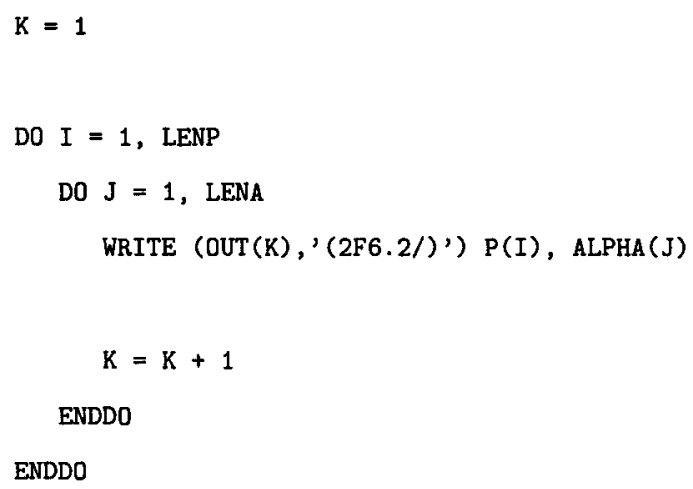

